$\mathrm{MZ}-\mathrm{TH} / 10-10$

HD-THEP-10-7

August 31, 2010

\title{
Renormalization-Group Improved Predictions for Top-Quark Pair Production at Hadron Colliders
}

\author{
Valentin Ahrens ${ }^{a}$, Andrea Ferroglia $^{a}$, Matthias Neubert $^{a, b}$, \\ BEN D. PECJAK ${ }^{a}$, AND LI LIN YANG ${ }^{a}$ \\ ${ }^{a}$ Institut für Physik (THEP), Johannes Gutenberg-Universität \\ D-55099 Mainz, Germany \\ ${ }^{b}$ Institut für Theoretische Physik, Ruprecht-Karls-Universität Heidelberg \\ Philosophenweg 16, D-69120 Heidelberg, Germany
}

\begin{abstract}
Precision predictions for phenomenologically interesting observables such as the $t \bar{t}$ invariant mass distribution and forward-backward asymmetry in top-quark pair production at hadron colliders require control over the differential cross section in perturbative QCD. In this paper we improve existing calculations of the doubly differential cross section in the invariant mass and scattering angle by using techniques from soft-collinear effective theory to perform an NNLL resummation of threshold logarithms, which become large when the invariant mass $M$ of the top-quark pair approaches the partonic center-of-mass energy $\sqrt{\hat{s}}$. We also derive an approximate formula for the differential cross section at NNLO in fixed-order perturbation theory, which completely determines the coefficients multiplying the singular plus distributions in the variable $\left(1-M^{2} / \hat{s}\right)$. We then match our results in the threshold region with the exact results at NLO in fixed-order perturbation theory, and perform a numerical analysis of the invariant mass distribution, the total cross section, and the forward-backward asymmetry. We argue that these are the most accurate predictions available for these observables at present. Using MSTW2008NNLO parton distribution functions (PDFs) along with $\alpha_{s}\left(M_{Z}\right)=0.117$ and $m_{t}=173.1 \mathrm{GeV}$, we obtain for the inclusive production cross sections at the Tevatron and LHC the values $\sigma_{\text {Tevatron }}=\left(6.30 \pm 0.19_{-0.23}^{+0.31}\right) \mathrm{pb}$ and $\sigma_{\mathrm{LHC}}=(149 \pm 7 \pm 8) \mathrm{pb}$, where the first error results from scale variations while the second reflects PDF uncertainties.
\end{abstract}




\section{Introduction}

The top quark is the heaviest known particle in the Standard Model (SM) of fundamental interactions. Because of its large mass, it is expected to couple strongly with the fields responsible for electroweak symmetry breaking, and the detailed study of top-quark properties is likely to play a key role in elucidating the origin of particle masses. The measurement of top-quark related observables is therefore one of the main goals of the Fermilab Tevatron and CERN Large Hadron Collider (LHC). To date, thousands of top-quark events have been observed by two different experiments at the Tevatron, and the top-quark mass has been extracted at the percent level [1]. The experiments at the LHC are expected to observe millions of top-quark events per year already in the initial low-luminosity phase, bringing the study of the top-quark properties into the realm of precision physics. In particular, the total inclusive cross section for top-quark pair production is expected to be measured with a relative error of $5 \%$ to $10 \%$ at the LHC [2]. In addition to the mass and the total inclusive cross section, kinematic distributions and charge asymmetries are also of interest. For instance, the $t \bar{t}$ invariant mass distribution can be used as a complementary method for measuring $m_{t}$ [3]. The presence of bumps in the smoothly decreasing $t \bar{t}$ invariant mass distribution would be a clear signal of an $s$-channel heavy resonance, which is predicted in many new physics scenarios [3-5]. Such searches have been pursued at the Tevatron [6-8], and results for the top-pair invariant mass distribution were recently obtained from data collected by the CDF collaboration [9]. The forward-backward asymmetry in $t \bar{t}$ production has also been measured recently [10-12] and shows a potential deviation from the theoretical predictions.

To make optimal use of the experimental measurements requires theoretical predictions of similar precision. At hadron colliders, a leading-order (LO) prediction is usually insufficient, and including higher-order corrections in QCD is mandatory. Current theoretical predictions for $t \bar{t}$ production in fixed-order perturbation theory are based on next-to-leading order (NLO) calculations of the total cross section [13-16], differential distributions [17-19], and the forward-backward asymmetry $[20,21]$. The NLO computations suffer from theoretical uncertainties larger than 10\%, both for Tevatron and LHC center-of-mass energies. These uncertainties are partly due to our imperfect knowledge of the parton distribution functions (PDFs), and partly to the truncation of the perturbative series in the strong coupling constant, which introduces a dependence on the unphysical renormalization and factorization scales into the physical predictions. The latter uncertainty is typically reduced by including more terms in the perturbative series, and for this reason the calculation of the partonic cross section to next-to-next-to-leading order (NNLO) has been an area of active research. This includes studies of the two-loop virtual corrections [22-26], the squared one-loop corrections [27-29], and the NLO corrections to the final state $t \bar{t}+$ jet [30]. However, due to the complexity of the calculations, complete results for the cross section at NNLO are not yet available.

In the absence of full NNLO results, one way to improve the NLO predictions is to utilize threshold resummation methods $[31,32]$ to incorporate some (presumably) dominant contributions from higher orders. For the total cross section, a common approach is to work in the limit where the top-quark pair is produced nearly at rest, and to resum logarithms in the velocity $\beta=\sqrt{1-4 m_{t}^{2} / \hat{s}} \rightarrow 0$, with $\hat{s}$ the partonic center-of-mass energy. Such resummations have been carried out at leading-logarithmic (LL) [33-38], next-to-leading-logarithmic 
(NLL) [39], and approximate next-to-next-to-leading-logarithmic (NNLL) order [40-42]. Only very recently have the complete expressions at NNLL order been obtained [43-45]. The calculation of differential cross sections is more involved due to the appearance of several kinematic variables. A typical approach in that case is to work in the threshold limit where the parameter $(1-z)=1-M^{2} / \hat{s} \rightarrow 0$, with $M$ the invariant mass of the $t \bar{t}$ pair, and to resum logarithms in $(1-z)$. In that case real gluon emission is soft, but the parameter $\beta$ is a generic $\mathcal{O}(1)$ variable. The current frontier for resummation in this limit is NLL calculations for differential cross sections [46-48] and the forward-backward asymmetry [49]. In addition, approximate NNLL calculations were performed in [50-53]. Extending these results to full NNLL order has been made possible by our recent calculation of the two-loop anomalous-dimension matrices $[54,55]$. We have presented an approximate NNLO formula for the $t \bar{t}$ invariant mass distribution in [56]. The goal of the present paper is to derive a renormalization-group (RG) improved expression for the doubly differential cross section at NNLL order, in which all threshold-enhanced terms are resummed. We will match this expression with the exact fixedorder NLO results and study the top-pair invariant mass distribution, the forward-backward asymmetry, and the total cross section at NLO+NNLL order. An important part of our analysis is a comparison of the total cross section obtained here with those obtained in the limit $\beta \rightarrow 0$. The outcome of this comparison leads us to conclude that the predictions obtained in this paper are the most precise available at present.

The paper is organized as follows. In Section 2 we review the kinematics and the structure of factorization in the threshold region. We then derive the factorization formula for the hard-scattering kernels into products of hard and soft matrices using soft-collinear effective theory (SCET) in Section 3. In Section 4 we present the calculation of the hard and soft matrices at NLO, and describe several checks on our results. Section 5 deals with the RG properties of the hard and soft functions. We derive a formula for the resummed cross section in momentum space using RG methods and describe its evaluation at NNLL order. We also review the derivation of the approximate NNLO formula, which has been presented first in [56]. In Section 6 we perform numerical studies of the invariant mass distribution, the total cross section, and the forward-backward asymmetry, utilizing both RG-improved perturbation theory at NNLL order and the NNLO approximate formula. This section includes the aforementioned comparison of different methods for obtaining the total cross section. We conclude in Section 7.

\section{Kinematics and factorization at threshold}

We consider the process

$$
N_{1}\left(P_{1}\right)+N_{2}\left(P_{2}\right) \rightarrow t\left(p_{3}\right)+\bar{t}\left(p_{4}\right)+X\left(p_{X}\right),
$$

where $X$ is an inclusive hadronic final state ${ }^{1}$. At Born level this proceeds through the $q \bar{q}$ annihilation and gluon-fusion channels

$$
q\left(p_{1}\right)+\bar{q}\left(p_{2}\right) \rightarrow t\left(p_{3}\right)+\bar{t}\left(p_{4}\right)
$$

\footnotetext{
${ }^{1}$ Throughout the analysis we treat the top-quarks as on-shell partons and neglect their decay. Corrections to this picture are suppressed by $\Gamma_{t} / m_{t} \ll 1$.
} 


$$
g\left(p_{1}\right)+g\left(p_{2}\right) \rightarrow t\left(p_{3}\right)+\bar{t}\left(p_{4}\right)
$$

where $p_{1}=x_{1} P_{1}$ and $p_{2}=x_{2} P_{2}$. We define the kinematic invariants

$$
\begin{aligned}
s & =\left(P_{1}+P_{2}\right)^{2}, \quad \hat{s}=\left(p_{1}+p_{2}\right)^{2}, \quad M^{2}=\left(p_{3}+p_{4}\right)^{2}, \\
t_{1} & =\left(p_{1}-p_{3}\right)^{2}-m_{t}^{2}, \quad u_{1}=\left(p_{2}-p_{3}\right)^{2}-m_{t}^{2},
\end{aligned}
$$

and momentum conservation at Born level implies $\hat{s}+t_{1}+u_{1}=0$.

In this section we consider the structure of the differential cross section near the partonic threshold. While the fully differential cross section depends on three kinematic variables, in this paper we are mainly interested in the doubly differential cross section expressed in terms of the invariant mass $M$ of the $t \bar{t}$ pair and the scattering angle $\theta$ between $\vec{p}_{1}$ and $\vec{p}_{3}$ in the partonic center-of-mass frame. To describe this distribution we introduce the variables

$$
z=\frac{M^{2}}{\hat{s}}, \quad \tau=\frac{M^{2}}{s}, \quad \beta_{t}=\sqrt{1-\frac{4 m_{t}^{2}}{M^{2}}} .
$$

The quantity $\beta_{t}$ gives the 3 -velocity of the top quarks in the $t \bar{t}$ rest frame. One often considers a related variable $\beta=\sqrt{1-4 m_{t}^{2} / \hat{s}}$, which coincides with $\beta_{t}$ in the limit $z \rightarrow 1$.

Before moving on, we would like to clarify our definition of the so-called "threshold region". In general, the definition of this region depends on the measured observable. For instance, to describe the total inclusive cross section, one often considers the limit $\beta \rightarrow 0$ [39-45]. To describe the $t \bar{t}$ invariant mass distribution, on the other hand, we consider the limit $z \rightarrow 1$, with $\beta_{t}$ a generic $\mathcal{O}(1)$ variable. In that case the top quarks are not produced at rest, nor are they highly boosted, and the emitted partons in the final state $X$ are constrained to be soft. This threshold limit is often referred to in the literature as the pair-invariant-mass kinematics, and the theoretical framework to deal with this situation was developed in $[46,47,57,58]$. A closely related framework can be applied to processes where only a single top-quark is observed [59,60], but will not be considered here. Threshold resummation at fixed invariant mass $M$ is completely analogous to the well-studied case of Drell-Yan or Higgs production, and it becomes more and more important as $M$ is increased. Therefore, the RG-improved result for $d \sigma / d M$ offers the best possible prediction for this observable. Moreover, integrating this distribution over $M$ then yields the best possible prediction for the total cross section. In particular, we believe that in this way we obtain a better description of the total cross section than the usual approach of resumming soft gluon and Coulomb terms for $\beta \rightarrow 0$. The reason is that at the Tevatron and LHC, the cross section is dominated by events with $\beta \approx 0.3-0.6$, which is not especially small. We will come back to this important point in Section 6.3.

According to the QCD factorization theorem [61], the differential cross section in $M$ and $\cos \theta$ can be written as

$$
\frac{d^{2} \sigma}{d M d \cos \theta}=\frac{8 \pi \beta_{t}}{3 s M} \sum_{i, j} \int_{\tau}^{1} \frac{d z}{z} f f_{i j}\left(\tau / z, \mu_{f}\right) C_{i j}\left(z, M, m_{t}, \cos \theta, \mu_{f}\right),
$$

where $\mu_{f}$ is the factorization scale, and the parton luminosity functions $f f_{i j}$ are defined by

$$
f f_{i j}\left(y, \mu_{f}\right)=\int_{y}^{1} \frac{d x}{x} f_{i / N_{1}}\left(x, \mu_{f}\right) f_{j / N_{2}}\left(y / x, \mu_{f}\right) .
$$


The $f_{i / N}$ are universal non-perturbative PDFs of the parton $i$ in the hadron $N$, which can be extracted from experimental data. The hard-scattering kernels $C_{i j}$ are related to the partonic cross sections and can be calculated as a power series in $\alpha_{s}$. We shall write their expansion as

$$
C_{i j}=\alpha_{s}^{2}\left[C_{i j}^{(0)}+\frac{\alpha_{s}}{4 \pi} C_{i j}^{(1)}+\left(\frac{\alpha_{s}}{4 \pi}\right)^{2} C_{i j}^{(2)}+\ldots\right] .
$$

At leading order in $\alpha_{s}$, only $C_{q \bar{q}}$ and $C_{g g}$ are non-zero. They are proportional to $\delta(1-z)$ and read

$$
\begin{aligned}
& C_{q \bar{q}}^{(0)}=\delta(1-z) \frac{3}{8 N} C_{F}\left(\frac{t_{1}^{2}+u_{1}^{2}}{M^{4}}+\frac{2 m_{t}^{2}}{M^{2}}\right), \\
& C_{g g}^{(0)}=\delta(1-z) \frac{3}{8\left(N^{2}-1\right)}\left(C_{F} \frac{M^{4}}{t_{1} u_{1}}-C_{A}\right)\left[\frac{t_{1}^{2}+u_{1}^{2}}{M^{4}}+\frac{4 m_{t}^{2}}{M^{2}}-\frac{4 m_{t}^{4}}{t_{1} u_{1}}\right],
\end{aligned}
$$

where $N=3$ is the number of colors in QCD, and $t_{1}$ and $u_{1}$ can be expressed in terms of $M$ and $\cos \theta$ as

$$
t_{1}=-\frac{M^{2}}{2}\left(1-\beta_{t} \cos \theta\right), \quad u_{1}=-\frac{M^{2}}{2}\left(1+\beta_{t} \cos \theta\right) .
$$

Note that for the doubly differential cross section the coefficient $C_{\bar{q} q}$ is also needed. It can be obtained from the expression for $C_{q \bar{q}}$ by replacing $\cos \theta \rightarrow-\cos \theta$, which is a symmetry at tree level but not beyond.

At higher orders in $\alpha_{s}$ the hard-scattering kernels receive corrections from virtual loop diagrams and real gluon emissions in the $q \bar{q}$ and $g g$ channels, as well as from other partonic channels such as $g q \rightarrow t \bar{t} q$. The calculation of these corrections near threshold is greatly simplified. For $z \rightarrow 1$ there is no phase-space available for hard gluon emission, which is thus suppressed by powers of $(1-z)$. Moreover, contributions from channels such as $g q \rightarrow t \bar{t} q$, which involve external soft-quark fields, are also suppressed. The partonic scattering process is thus dominated by virtual corrections and the real emission of soft gluons. The phase-space for such processes is effectively that for a two-body final state, so the hard-scattering kernels can be written in terms of the kinematic invariants from the Born-level processes. Therefore, up to corrections of order $(1-z)$, we can rewrite (5) in the threshold region as

$$
\begin{aligned}
& \frac{d^{2} \sigma}{d M d \cos \theta}=\frac{8 \pi \beta_{t}}{3 s M} \int_{\tau}^{1} \frac{d z}{z}\left[f f_{g g}\left(\tau / z, \mu_{f}\right) C_{g g}\left(z, M, m_{t}, \cos \theta, \mu_{f}\right)\right. \\
& \left.\quad+\int f_{q \bar{q}}\left(\tau / z, \mu_{f}\right) C_{q \bar{q}}\left(z, M, m_{t}, \cos \theta, \mu_{f}\right)+f f_{\bar{q} q}\left(\tau / z, \mu_{f}\right) C_{q \bar{q}}\left(z, M, m_{t},-\cos \theta, \mu_{f}\right)\right],
\end{aligned}
$$

where $\int f_{q \bar{q}}$ is understood to be summed over all light quark flavors.

In the threshold limit $z \rightarrow 1$, the hard-scattering kernels $C_{i j}$ can be factorized into a product of hard and soft functions according to

$$
C_{i j}\left(z, M, m_{t}, \cos \theta, \mu_{f}\right)=\operatorname{Tr}\left[\boldsymbol{H}_{i j}\left(M, m_{t}, \cos \theta, \mu_{f}\right) \boldsymbol{S}_{i j}\left(\sqrt{\hat{s}}(1-z), m_{t}, \cos \theta, \mu_{f}\right)\right]+\mathcal{O}(1-z) .
$$


The boldface indicates that the hard functions $\boldsymbol{H}_{i j}$ and soft functions $\boldsymbol{S}_{i j}$ are matrices in color space, with respect to which the trace is taken. We will derive this formula in the next section, using techniques from SCET. (A similar factorization formula in Mellin moment space was derived in [47].) The hard functions are related to the virtual corrections and are ordinary functions of their arguments, while the soft functions are related to the real emission of soft gluons and contain singular distributions in $(1-z)$. In addition to terms proportional to $\delta(1-z)$, the $n$-th order corrections in $\alpha_{s}$ also contain plus distributions of the form

$$
\left[\frac{\ln ^{m}(1-z)}{1-z}\right]_{+} ; \quad m=0, \ldots, 2 n-1
$$

where

$$
\int_{\tau}^{1} d z\left[\frac{\ln ^{m}(1-z)}{1-z}\right]_{+} g(z)=\int_{\tau}^{1} d z \frac{\ln ^{m}(1-z)}{1-z}[g(z)-g(1)]-g(1) \int_{0}^{\tau} d z \frac{\ln ^{m}(1-z)}{1-z}
$$

for an arbitrary function $g(z)$. These singular distributions make the perturbative series badly convergent near threshold and must be resummed to all orders in perturbation theory. In this paper we perform such a resummation directly in momentum space [62], up to NNLL order. To this end, we extend the procedure for deep-inelastic scattering [63], Drell-Yan process [64], Higgs production [65,66], and direct photon production [67] to processes with four colored external particles. The formalism will be described in Section 5.

\section{Factorization at threshold in SCET}

In this section we derive the factorization formula (10) for the hard-scattering kernels in the threshold region using SCET [68-70] and heavy-quark effective theory (HQET) (for a review, see [71]). The derivation is similar to the ones in $[63,64,67]$, but is more complicated due to the presence of additional Dirac and color structures. The derivation of factorization in the effective theory relies on a two-step matching procedure. In the first step, fluctuations at the hard scale from virtual corrections are integrated out by matching QCD onto an effectivetheory with collinear and soft degrees of freedom. The Wilson coefficients from this matching step give the hard function when squaring the amplitude. In the second step, the soft degrees of freedom are integrated out, giving rise to a soft function, which is defined as the vacuum expectation value of a Wilson loop operator.

\subsection{Fields and operators}

The scattering amplitude for $t \bar{t}$ production involves several scales, which we assume to satisfy

$$
\hat{s}, M^{2},\left|t_{1}\right|,\left|u_{1}\right|, m_{t}^{2} \gg \hat{s}(1-z)^{2} \gg \Lambda_{\mathrm{QCD}}^{2}
$$

in the threshold region. The elements of the first set of scales are taken to be of the same order and shall be collectively referred to as hard scales, whereas $\hat{s}(1-z)^{2}$ defines the soft scale. The small quantity $\lambda=(1-z) \ll 1$ then serves as the expansion parameter in the effective theory. 
Note that we treat $M$ and $m_{t}$ as of the same order, which means that the top quarks are not highly boosted. To describe the invariant mass spectrum in the region where $M \gg 2 m_{t}$, a more appropriate treatment would require a different effective theory to separate these two scales, and two jet functions have to be introduced for the top and anti-top quarks. Such an approach was adopted in [72] for top-quark production in $e^{+} e^{-}$collisions, where $m_{t} / M$ was used as a small expansion parameter. However, given that even for $M$ as large as $1.5 \mathrm{TeV}$ the ratio $2 m_{t} / M \approx 0.23$ is still a reasonable $\mathcal{O}(1)$ parameter, we see no need to adopt it for the present work.

The formalism for SCET applied to a generic $n$-body scattering process involving both heavy and light partons was set up in $[73,74]$. In our case, the effective theory contains two sets of collinear fields to describe the degrees of freedom in the incoming hadrons, two sets of HQET fields to describe the outgoing heavy quarks, and a single set of soft fields describing the final state $X$ and the soft interactions among particles. In classifying the collinear fields we define two light-like vectors $n$ and $\bar{n}$ in the directions of the colliding partons, which satisfy $n \cdot \bar{n}=2$. The collinear quark fields are related to the QCD fields by

$$
\xi_{n}(x)=\frac{\not h \hbar}{4} \psi(x), \quad \xi_{\bar{n}}(x)=\frac{\not h \hbar}{4} \psi(x) .
$$

The collinear gluon fields in a single collinear sector are identical to those in QCD, with their momenta restricted to be collinear to the given direction. In constructing operators below, it will be convenient to introduce the manifestly gauge-invariant combinations of fields $[69,75]$

$$
\chi_{n}(x)=W_{n}^{\dagger}(x) \xi_{n}(x), \quad \mathcal{A}_{n \perp}^{\mu}(x)=W_{n}^{\dagger}(x)\left[i D_{\perp}^{\mu} W_{n}(x)\right],
$$

where the $n$-collinear Wilson line is defined by

$$
W_{n}(x)=\mathcal{P} \exp \left(i g \int_{-\infty}^{0} d s \bar{n} \cdot A_{n}(x+s \bar{n})\right),
$$

and $\mathcal{P}$ denotes path ordering. The corresponding objects for the $\bar{n}$-collinear fields are obtained by interchanging $n$ and $\bar{n}$. The HQET fields $h_{v_{3}}$ and $h_{v_{4}}$ are labeled by the velocities of the top quark and anti-quark, which are related to their momenta as

$$
p_{3}^{\mu}=m_{t} v_{3}^{\mu}+k_{3}^{\mu}, \quad p_{4}^{\mu}=m_{t} v_{4}^{\mu}+k_{4}^{\mu} .
$$

The residual momenta $k_{i}$ scale as soft momenta and are set to zero for on-shell quarks. In terms of the QCD top-quark fields, the HQET fields are defined as

$$
h_{v_{i}}(x)=\frac{1+\psi}{2} e^{-i m_{t} v_{i} \cdot x} t(x) .
$$

A crucial property of the leading-order SCET Lagrangian is that the interactions of soft gluon fields with collinear and heavy-quark fields are described by eikonal vertices. The explicit form of the interaction terms for soft gluons with the fermion fields is

$$
\mathcal{L}_{\mathrm{int}}=\bar{\xi}_{n}(x) \frac{\not h}{2} g n \cdot A_{s}(x) \xi_{n}(x)+\bar{\xi}_{\bar{n}}(x) \frac{\not h}{2} g \bar{n} \cdot A_{s}(x) \xi_{\bar{n}}(x)
$$




$$
+\bar{h}_{v_{3}}(x) g v_{3} \cdot A_{s}(x) h_{v_{3}}(x)+\bar{h}_{v_{4}}(x) g v_{4} \cdot A_{s}(x) h_{v_{4}}(x),
$$

and those between collinear and soft gluon fields can be deduced by making the substitution $A_{n} \rightarrow A_{n}+n \cdot A_{s} \bar{n} / 2$ (and similarly for the $\bar{n}$-collinear fields) in the Yang-Mills Lagrangian. Such eikonal interactions can be absorbed into Wilson lines via the field redefinitions $[69,76]$

$$
\chi_{n}^{a}(x) \rightarrow\left[S_{n}(x)\right]^{a b} \chi_{n}^{b(0)}(x), \quad \mathcal{A}_{n \mu}^{a}(x) \rightarrow\left[S_{n}^{\text {adj }}(x)\right]^{a b} \mathcal{A}_{n \mu}^{b(0)}(x), \quad h_{v_{3}}^{a}(x) \rightarrow\left[S_{v_{3}}(x)\right]^{a b} h_{v_{3}}^{b(0)}(x),
$$

with

$$
\begin{aligned}
{\left[S_{n}(x)\right]^{a b} } & =\mathcal{P} \exp \left(i g \int_{-\infty}^{0} d t n \cdot A_{s}^{c}(x+t n) t_{a b}^{c}\right) \\
{\left[S_{n}^{\operatorname{adj}}(x)\right]^{a b} } & =\mathcal{P} \exp \left(i g \int_{-\infty}^{0} d t n \cdot A_{s}^{c}(x+t n)\left(-i f^{c a b}\right)\right), \\
{\left[S_{v_{3}}(x)\right]^{a b} } & =\mathcal{P} \exp \left(-i g \int_{0}^{\infty} d t v_{3} \cdot A_{s}^{c}\left(x+t v_{3}\right) t_{a b}^{c}\right),
\end{aligned}
$$

and similarly for the $\bar{n}$-collinear and $h_{v_{4}}$ fields. We have used the superscript "adj" to indicate Wilson lines in the adjoint representation. The fields with the superscript (0) no longer interact with soft gluon fields. Here $t^{c}$ are Gell-Mann matrices and $f^{c a b}$ are structure constants of QCD.

One must supplement the effective Lagrangian with a set of operators describing the $(q \bar{q}, g g) \rightarrow t \bar{t}$ scattering processes. These operators appear in the effective Hamiltonian in convolutions along light-like directions with perturbative Wilson coefficients arising from integrating out hard virtual fluctuations. We write this effective Hamiltonian as

$$
\mathcal{H}_{\mathrm{eff}}(x)=\sum_{I, m} \int d t_{1} d t_{2} e^{i m_{t}\left(v_{3}+v_{4}\right) \cdot x}\left[\tilde{C}_{I m}^{q \bar{q}}\left(t_{1}, t_{2}\right) O_{I m}^{q \bar{q}}\left(x, t_{1}, t_{2}\right)+\tilde{C}_{I m}^{g g}\left(t_{1}, t_{2}\right) O_{I m}^{g g}\left(x, t_{1}, t_{2}\right)\right],
$$

where $I$ labels color structures and $m$ labels Dirac structures. The operators can be written as

$$
\begin{aligned}
& O_{I m}^{q \bar{q}}\left(x, t_{1}, t_{2}\right)=\sum_{\{a\}}\left(c_{I}^{q \bar{q}}\right)_{\{a\}} \bar{\chi}_{\bar{n}}^{a_{2}}\left(x+t_{2} n\right) \Gamma_{m}^{\prime} \chi_{n}^{a_{1}}\left(x+t_{1} \bar{n}\right) \bar{h}_{v_{3}}^{a_{3}}(x) \Gamma_{m}^{\prime \prime} h_{v_{4}}^{a_{4}}(x), \\
& O_{I m}^{g g}\left(x, t_{1}, t_{2}\right)=\sum_{\{a\}}\left(c_{I}^{g g}\right)_{\{a\}} \mathcal{A}_{n \mu \perp}^{a_{1}}\left(x+t_{1} \bar{n}\right) \mathcal{A}_{\bar{n} \nu \perp}^{a_{2}}\left(x+t_{2} n\right) \bar{h}_{v_{3}}^{a_{3}}(x) \Gamma_{m}^{\mu \nu} h_{v_{4}}^{a_{4}}(x),
\end{aligned}
$$

where $\Gamma_{m}^{\mu \nu}, \Gamma_{m}^{\prime}$, and $\Gamma_{m}^{\prime \prime}$ are combinations of Dirac matrices and the external vectors $n, \bar{n}, v_{3}$, and $v_{4}$ (note that there can be contractions of Lorentz indices between $\Gamma_{m}^{\prime}$ and $\Gamma_{m}^{\prime \prime}$ ). The $c_{I}^{q \bar{q}}$ and $c_{I}^{g g}$ are tensors in color space, whose indices $\{a\} \equiv\left\{a_{1}, a_{2}, a_{3}, a_{4}\right\}$ can be in either the fundamental or adjoint representation. For each channel, they are chosen to be the independent 
color singlet (e.g. gauge-invariant) structures needed to describe the scattering amplitude. We will choose the color structures to be in the singlet-octet bases

$$
\begin{gathered}
\left(c_{1}^{q \bar{q}}\right)_{\{a\}}=\delta_{a_{1} a_{2}} \delta_{a_{3} a_{4}}, \quad\left(c_{2}^{q \bar{q}}\right)_{\{a\}}=t_{a_{2} a_{1}}^{c} t_{a_{3} a_{4}}^{c}, \\
\left(c_{1}^{g g}\right)_{\{a\}}=\delta^{a_{1} a_{2}} \delta_{a_{3} a_{4}}, \quad\left(c_{2}^{g g}\right)_{\{a\}}=i f^{a_{1} a_{2} c} t_{a_{3} a_{4}}^{c}, \quad\left(c_{3}^{g g}\right)_{\{a\}}=d^{a_{1} a_{2} c} t_{a_{3} a_{4}}^{c} .
\end{gathered}
$$

When squaring the amplitude and summing over colors, one must evaluate products of the color structures with their indices contracted. In the absence of soft gluon emissions, these are of the form $\left(c_{I}^{q \bar{q}}\right)_{a_{1} a_{2} a_{3} a_{4}}\left(c_{J}^{q \bar{q}}\right)_{a_{1} a_{2} a_{3} a_{4}}^{*}$ (and similarly for the gluon fusion channel) and are equal to an $N$-dependent factor multiplying $\delta_{I J}$. In this sense the color structures are orthogonal, but not orthonormal.

Time-ordered products of the operators (24) with the SCET and HQET Lagrangians describe the collinear and soft contributions to the $(q \bar{q}, g g) \rightarrow t \bar{t} X$ scattering amplitudes in QCD, where the final state $X$ contains any number of soft gluons from real emissions. In the formulation used so far, the final state $X$ is built up through insertions of the interaction Lagrangian (20) into the SCET operators. To account explicitly for soft gluon emission to all orders in the strong coupling constant, it is convenient to use the decoupling relations (21) and represent the soft gluon interactions by Wilson lines. Performing this decoupling and dropping the superscripts on the new fields, the operators factorize into products of collinear, heavy-quark, and soft-gluon operators in the form of Wilson loops. The resulting operators in the $q \bar{q}$ channel read

$$
O_{I m}\left(x, t_{1}, t_{2}\right)=\sum_{\{a\},\{b\}}\left(c_{I}\right)_{\{a\}}\left[O_{m}^{h}(x)\right]^{b_{3} b_{4}}\left[O_{m}^{c}\left(x, t_{1}, t_{2}\right)\right]^{b_{1} b_{2}}\left[O^{s}(x)\right]^{\{a\},\{b\}},
$$

where

$$
\begin{gathered}
{\left[O_{m}^{h}(x)\right]^{b_{3} b_{4}}=\bar{h}_{v_{3}}^{b_{3}}(x) \Gamma_{m}^{\prime \prime} h_{v_{4}}^{b_{4}}(x), \quad\left[O_{m}^{c}\left(x, t_{1}, t_{2}\right)\right]^{b_{1} b_{2}}=\bar{\chi}_{\bar{n}}^{b_{2}}\left(x+t_{2} n\right) \Gamma_{m}^{\prime} \chi_{n}^{b_{1}}\left(x+t_{1} \bar{n}\right)} \\
{\left[O^{s}(x)\right]^{\{a\},\{b\}}=\left[S_{v_{3}}^{\dagger}(x)\right]^{b_{3} a_{3}}\left[S_{v_{4}}(x)\right]^{a_{4} b_{4}}\left[S_{\bar{n}}^{\dagger}(x)\right]^{b_{2} a_{2}}\left[S_{n}(x)\right]^{a_{1} b_{1}}}
\end{gathered}
$$

Those in the gluon-fusion channel are obtained by making the obvious replacements in Dirac structure and the collinear operators, and by changing the Wilson lines $S_{n}$ and $S_{\bar{n}}$ to the corresponding ones in the adjoint representation.

In the form shown above, the different sectors no longer interact with each other. After squaring the amplitude, this property leads to the factorized form (10) for the hard-scattering kernels. A complication is that the color indices on the Wilson lines representing the soft-gluon interactions act on the color structures (25) and can mix them into each other. Consider, for example, the calculation of an $\mathcal{O}\left(\alpha_{s}\right)$ correction due to soft gluon exchange between partons 1 and 3 in the squared amplitude in the $q \bar{q}$ channel. This could either be from the product of diagrams involving the real emission of one soft gluon, or from a virtual diagram with a soft loop (which would be scaleless for on-shell quarks, but appears in the calculation of the anomalous-dimension matrix). After summing over colors one must evaluate contractions of the form

$$
\left(c_{I}^{q \bar{q}}\right)_{\left\{b_{1} a_{2} b_{3} a_{4}\right\}} t_{b_{1} a_{1}}^{c} t_{a_{3} b_{3}}^{c}\left(c_{J}^{q \bar{q}}\right)_{\left\{a_{1} a_{2} a_{3} a_{4}\right\}}^{*} \cdot
$$


In general, this contraction is not proportional to $\delta_{I J}$, and must be worked out case by case. This mixing of the different color structures due to soft gluon exchange is responsible for the non-trivial matrix structure of the hard and soft functions. To organize this color algebra, it is convenient to use the color-space formalism $[77,78]$. Before moving on to the calculation of the differential cross section in Section 3.3, we briefly pause to review this formalism.

\subsection{Color-space formalism}

Consider the on-shell scattering amplitudes for $(q \bar{q}, g g) \rightarrow t \bar{t}$, for a given color configuration of the external particles. We write this in the quark channel as

$$
\mathcal{M}_{\{a\}}^{q \bar{q}}=\left\langle t^{a_{3}}\left(p_{3}\right) \bar{t}^{a_{4}}\left(p_{4}\right)\left|\mathcal{H}_{\mathrm{eff}}(0)\right| q^{a_{1}}\left(p_{1}\right) \bar{q}^{a_{2}}\left(p_{2}\right)\right\rangle,
$$

and also define the object $\mathcal{M}_{\{a\}}^{g g}$ in the obvious way. In what follows, we will drop the superscript indicating the channel, and work with a single amplitude which can represent either $\mathcal{M}^{q \bar{q}}$ or $\mathcal{M}^{g g}$. As in $[77,78]$, we introduce an orthonormal basis of vectors $\left\{\left|a_{1}, a_{2}, a_{3}, a_{4}\right\rangle\right\}$, where the indices $\{a\}$ refer to the colors of the external particles. The amplitude can then be written as

$$
\mathcal{M}_{\{a\}}=\left\langle a_{1}, a_{2}, a_{3}, a_{4} \mid \mathcal{M}\right\rangle,
$$

where the object $|\mathcal{M}\rangle$ is an abstract vector in color space. Since we only consider color-singlet amplitudes, we can decompose the QCD amplitude into the set of color structures (25). In the color-space formalism, this is done by writing

$$
|\mathcal{M}\rangle=\sum_{I} \mathcal{M}_{I} \sum_{\{a\}}\left(c_{I}\right)_{\{a\}}|\{a\}\rangle \equiv \mathcal{M}_{I}\left|c_{I}\right\rangle
$$

where the coefficients $\mathcal{M}_{I}$ are combinations of Dirac matrices, external vectors, spinors, and polarization vectors. Note that with this definition the basis vectors $\left|c_{I}\right\rangle$ are orthogonal but not normalized, so to project out the $\mathcal{M}_{I}$ one must use

$$
\mathcal{M}_{I}=\frac{1}{\left\langle c_{I} \mid c_{I}\right\rangle}\left\langle c_{I} \mid \mathcal{M}\right\rangle \text {. }
$$

The square of the amplitude summed over colors is then given by the inner product of $|\mathcal{M}\rangle$ :

$$
\sum_{\text {colors }}|\mathcal{M}|^{2}=\langle\mathcal{M} \mid \mathcal{M}\rangle=\sum_{I} \mathcal{M}_{I}^{*} \mathcal{M}_{I}\left\langle c_{I} \mid c_{I}\right\rangle=\sum_{I} \sum_{\{a\}} \mathcal{M}_{I}^{*} \mathcal{M}_{I}\left(c_{I}\right)_{a_{1} a_{2} a_{3} a_{4}}^{*}\left(c_{I}\right)_{a_{1} a_{2} a_{3} a_{4}}
$$

Following $[77,78]$, we introduce color generators $\boldsymbol{T}_{i}$ to describe the color algebra associated with the emission of a soft gluon from parton $i=1,2,3,4$. These matrices act on the color indices of the $i$-th parton as

$$
\boldsymbol{T}_{i}^{c}\left|\ldots, a_{i}, \ldots\right\rangle=\left(\boldsymbol{T}_{i}^{c}\right)_{b_{i} a_{i}}\left|\ldots, b_{i}, \ldots\right\rangle .
$$

If the $i$-th parton is a final-state quark or an initial-state anti-quark we set $\left(\boldsymbol{T}_{i}^{c}\right)_{b a}=t_{b a}^{c}$, for a final-state anti-quark or an initial-state quark we have $\left(\boldsymbol{T}_{i}^{c}\right)_{b a}=-t_{a b}^{c}$, and for a gluon we use 
$\left(\boldsymbol{T}_{i}^{c}\right)_{b a}=i f f^{a b c}$. We also use the notation $\boldsymbol{T}_{i} \cdot \boldsymbol{T}_{j} \equiv \boldsymbol{T}_{i}^{c} \boldsymbol{T}_{j}^{c}$, and $\boldsymbol{T}_{i}^{2}$ denotes the quadratic Casimir operator in the representation of the $i$-th parton, with eigenvalues $C_{F}$ for quarks and $C_{A}$ for gluons. Since we consider color-singlet amplitudes, color conservation implies the relation

$$
\left(\boldsymbol{T}_{1}+\boldsymbol{T}_{2}+\boldsymbol{T}_{3}+\boldsymbol{T}_{4}\right)|\mathcal{M}\rangle=0 .
$$

We will be particularly interested in products of color generators acting on the scattering amplitudes, which appear in the calculation of perturbative corrections to the differential cross section. We write such products as, for instance,

$$
\left\langle\mathcal{M}\left|\boldsymbol{T}_{2} \cdot \boldsymbol{T}_{4}\right| \mathcal{M}\right\rangle=\mathcal{M}_{a_{1} b_{2} a_{3} b_{4}}^{*}\left(\boldsymbol{T}_{2}^{c}\right)_{b_{2} a_{2}}\left(\boldsymbol{T}_{4}^{c}\right)_{b_{4} a_{4}} \mathcal{M}_{a_{1} a_{2} a_{3} a_{4}}
$$

Rather than evaluating such expressions for each amplitude $\mathcal{M}$, it is more convenient to work out how the products $\boldsymbol{T}_{i} \cdot \boldsymbol{T}_{j}$ act on the basis vectors $\left|c_{I}\right\rangle$ :

$$
\boldsymbol{T}_{i} \cdot \boldsymbol{T}_{j}\left|c_{J}\right\rangle=\left[\boldsymbol{T}_{i} \cdot \boldsymbol{T}_{j}\right]_{I J}\left|c_{I}\right\rangle,
$$

where the matrix elements are given by

$$
\left[\boldsymbol{T}_{i} \cdot \boldsymbol{T}_{j}\right]_{I J}=\frac{1}{\left\langle c_{I} \mid c_{I}\right\rangle}\left\langle c_{I}\left|\boldsymbol{T}_{i} \cdot \boldsymbol{T}_{j}\right| c_{J}\right\rangle
$$

It is worth emphasizing that while the generators themselves act in the abstract color space, on the left-hand side of the above equation they are just labels to identify a matrix acting in the space of color-singlet structures. This matrix is thus a $2 \times 2$ matrix for the $q \bar{q}$ channel, and a $3 \times 3$ matrix for the $g g$ channel.

We now consider the SCET representation of the amplitude $\mathcal{M}_{\{a\}}$. For the $q \bar{q}$ channel, this is equal to

$$
\begin{aligned}
& \sum_{I, m} \int d t_{1} d t_{2} \tilde{C}_{I m}\left(t_{1}, t_{2}\right)\left(c_{I}\right)_{\{a\}} \\
& \quad \times\left\langle t^{b_{3}}\left(p_{3}\right) \bar{t}^{b_{4}}\left(p_{4}\right)\left|\left[O_{m}^{h}\right]^{b_{3} b_{4}}\left[O_{m}^{c}\right]^{b_{1} b_{2}}\left[O^{s}\right]^{\{a\},\{b\}}\left(0, t_{1}, t_{2}\right)\right| q^{b_{1}}\left(p_{1}\right) \bar{q}^{b_{2}}\left(p_{2}\right)\right\rangle,
\end{aligned}
$$

where no summation over the set of indices $\{b\}$ is performed. We have made clear that for the amplitude to be non-zero, the colors of the heavy-quark and collinear fields must coincide with those of the external partons. The reason is that after the decoupling of soft gluons, the heavy-quark fields are effectively free fields, while collinear exchanges take place only within $O_{m}^{c}$ itself and are diagonal in color space. We can therefore suppress the color indices on $O_{m}^{h}$ and $O_{m}^{c}$ as well as on the external states, and keep in mind that we shall sum over the color indices of $O^{s}$ when we square the amplitude. Color correlations such as (36) are mediated by the exchange of soft gluons, which are represented by the Wilson lines in the soft operator $O_{s}$. To describe these exchanges in the color-space formalism we use the operator

$$
\boldsymbol{O}_{s}(x)=\left[\boldsymbol{S}_{n} \boldsymbol{S}_{\bar{n}}^{\dagger} \boldsymbol{S}_{v_{3}}^{\dagger} \boldsymbol{S}_{v_{4}}\right](x),
$$

where the Wilson lines $\boldsymbol{S}_{i}$ are defined as in (22), with the color generators promoted to the abstract ones $\boldsymbol{T}_{i}$ according to the rules stated below in (34). The decoupling relations (21) 
are of the same form for quarks and gluons when expressed in terms of the $\boldsymbol{S}_{i}$ [79], so this operator is used for both the $q \bar{q}$ and $g g$ channels. Its action on the basis vectors $\left|c_{I}\right\rangle$ is then defined according to (36). We define the full SCET operator as $\boldsymbol{O}_{m}=O_{m}^{h} O_{m}^{c} \boldsymbol{O}^{s}$.

To evaluate the partonic matrix elements of the collinear fields we use

$$
\begin{aligned}
\left\langle 0\left|\left(\chi_{n}\right)_{\alpha}^{a}(t \bar{n})\right| p_{i} ; a_{i}, s_{i}\right\rangle & =\delta_{a a_{i}} e^{-i t \bar{n} \cdot p} u_{\alpha}\left(p_{i}, s_{i}\right), \\
\left\langle 0\left|\left(\mathcal{A}_{n \perp}\right)_{\alpha}^{a}(t \bar{n})\right| p_{i} ; a_{i}, s_{i}\right\rangle & =\delta_{a a_{i}} e^{-i t \bar{n} \cdot p} \epsilon_{\alpha}\left(p_{i}, s_{i}\right) .
\end{aligned}
$$

The heavy-quark fields are always taken to be on-shell, so their partonic matrix elements are equal to HQET spinors multiplied by Kronecker delta symbols in the spin and color indices. Upon taking the partonic matrix elements, the integrals over $t_{1}$ and $t_{2}$ produce the Fouriertransformed Wilson coefficients

$$
C_{I m}\left(M, m_{t}, \cos \theta, \mu\right)=\int d t_{1} d t_{2} e^{-i t_{1} \bar{n} \cdot p_{1}-i t_{2} n \cdot p_{2}} \tilde{C}_{I m}\left(t_{1}, t_{2}\right),
$$

where we have made the full dependence of the momentum space Wilson coefficients on the kinematic variables explicit. Defining a vector of Wilson coefficients as

$$
\left|C_{m}\right\rangle \equiv \sum_{I} C_{I m}\left|c_{I}\right\rangle
$$

and introducing the symbol $\langle\langle.\rangle$.$\rangle for partonic matrix elements as in [55], i.e.,$

$$
\left\langle\left\langle\boldsymbol{O}_{m}\right\rangle\right\rangle=\left\langle t\left(p_{3}\right) \bar{t}\left(p_{4}\right)\left|\boldsymbol{O}_{m}(0,0,0)\right| q\left(p_{1}\right) \bar{q}\left(p_{2}\right)\right\rangle,
$$

we can write the color-space representation of the SCET scattering amplitude as

$$
|\mathcal{M}\rangle=\sum_{m}\left\langle\left\langle\boldsymbol{O}_{m}\right\rangle\right\rangle\left|C_{m}\right\rangle
$$

\subsection{The differential cross section}

We now return to the derivation of the factorization formula (10) by calculating the partonic cross sections. The hadronic cross section is then obtained by convoluting these results with the PDFs. Below we will discuss the $q \bar{q}$ case in detail; the $g g$ channel can be analyzed in an analogous way.

The differential cross section is given by the phase-space integral of the squared amplitude

$$
\begin{aligned}
d \hat{\sigma}= & \frac{1}{2 \hat{s}} \frac{d^{3} \vec{p}_{3}}{(2 \pi)^{3} 2 E_{3}} \frac{d^{3} \vec{p}_{4}}{(2 \pi)^{3} 2 E_{4}} \sum_{X_{s}}(2 \pi)^{4} \delta^{(4)}\left(p_{s}+p_{3}+p_{4}-p_{1}-p_{2}\right) \\
& \left.\times \frac{1}{4 d_{R}^{2}}\left|\sum_{m}\left\langle t\left(p_{3}\right) \bar{t}\left(p_{4}\right) X_{s}\left(p_{s}\right)\left|\boldsymbol{O}_{m}(0)\right| q\left(p_{1}\right) \bar{q}\left(p_{2}\right)\right\rangle\right| C_{m}\right\rangle\left.\right|^{2},
\end{aligned}
$$

where $\boldsymbol{O}_{m}(x) \equiv \boldsymbol{O}_{m}(x, 0,0)$, and we have used translational invariance to write the result in terms of the Fourier-transformed coefficients $C_{m}$ from (42). The cross section is implicitly 
summed over the external colors and spins, and the factor $d_{R}=N$ for quarks and $d_{R}=N^{2}-1$ for gluons arises from averaging over the colors of the initial-state partons. The factor $1 / 4$ accounts for the averaging over the polarizations of the initial-state partons. Since the different types of effective-theory fields do not interact with each other, we can factorize the matrix element into soft, heavy-quark, and collinear pieces. The partonic matrix elements of the heavy-quark and collinear pieces just give the usual products of spinors, which we combine into the tree-level matrix element

$$
\left\langle\left\langle O_{m}\right\rangle\right\rangle_{\text {tree }} \equiv\left\langle\left\langle O_{m}^{h}(0) O_{m}^{c}(0)\right\rangle\right\rangle_{\text {tree }}=\left\langle t\left(p_{3}\right) \bar{t}\left(p_{4}\right)\left|O_{m}^{h}(0) O_{m}^{c}(0)\right| q\left(p_{1}\right) \bar{q}\left(p_{2}\right)\right\rangle_{\text {tree }} .
$$

Summed over spins, these give rise to the usual Dirac traces. The matrix element of the soft operator is taken using the vacuum as the initial state and is of the form $\left|\left\langle X_{s}\left(p_{s}\right)\left|\boldsymbol{O}_{s}(0)\right| 0\right\rangle\right|^{2}$. This can be evaluated directly, but we prefer instead to sum over the final states $X_{s}$ and convert it into a forward matrix element using the formalism explained in Appendix C of [64]. Written in this form, the differential cross section reads

$$
\begin{aligned}
d \hat{\sigma}= & \frac{1}{2 \hat{s}} \frac{d^{3} \vec{p}_{3}}{(2 \pi)^{3} 2 E_{3}} \frac{d^{3} \vec{p}_{4}}{(2 \pi)^{3} 2 E_{4}} \int d^{4} x e^{i\left(p_{1}+p_{2}-p_{3}-p_{4}\right) \cdot x} \\
& \times \frac{1}{4 d_{R}^{2}} \sum_{m, m^{\prime}}\left[\left\langle\left\langle O_{m}\right\rangle\right\rangle_{\text {tree }}^{\dagger}\left\langle\left\langle O_{m^{\prime}}\right\rangle\right\rangle_{\text {tree }} \times\left\langle C_{m}\left|\left\langle 0\left|\overline{\mathbf{T}}\left[\boldsymbol{O}^{s \dagger}(x)\right] \mathbf{T}\left[\boldsymbol{O}^{s}(0)\right]\right| 0\right\rangle\right| C_{m^{\prime}}\right\rangle\right],
\end{aligned}
$$

where $\mathbf{T}$ and $\overline{\mathbf{T}}$ represent time and anti-time ordering [64]. Since we treat the soft scale as perturbative, the soft fields can be integrated out by evaluating the vacuum matrix element. We now define a hard matrix and a position-space soft function as

$$
\begin{aligned}
\boldsymbol{H}\left(M, m_{t}, \cos \theta, \mu\right) & =\frac{3}{8} \frac{1}{(4 \pi)^{2}} \frac{1}{4 d_{R}} \sum_{m, m^{\prime}}\left\langle\left\langle O_{m^{\prime}}\right\rangle\right\rangle_{\text {tree }}\left|C_{m^{\prime}}\right\rangle\left\langle C_{m}\right|\left\langle\left\langle O_{m}\right\rangle\right\rangle_{\text {tree }}^{\dagger}, \\
\boldsymbol{W}(x, \mu) & =\frac{1}{d_{R}}\left\langle 0\left|\overline{\mathbf{T}}\left[\boldsymbol{O}^{s \dagger}(x)\right] \mathbf{T}\left[\boldsymbol{O}^{s}(0)\right]\right| 0\right\rangle,
\end{aligned}
$$

where we have chosen the prefactors to match the overall normalization of (10). The elements of these matrices in the chosen color basis are defined as

$$
H_{I J} \equiv \frac{1}{\left\langle c_{I} \mid c_{I}\right\rangle\left\langle c_{J} \mid c_{J}\right\rangle}\left\langle c_{I}|\boldsymbol{H}| c_{J}\right\rangle, \quad W_{I J} \equiv\left\langle c_{I}|\boldsymbol{W}| c_{J}\right\rangle
$$

so that the term in square brackets in the second line of (48) is proportional to

$$
\operatorname{Tr}[\boldsymbol{H} \boldsymbol{W}]=\sum_{I, J} H_{I J} W_{J I}
$$

In order to compute the invariant mass spectrum for the $t \bar{t}$ pair, we define $q=p_{3}+p_{4}$ and insert

$$
1=\int d^{4} q d M \delta^{(4)}\left(q-p_{3}-p_{4}\right) 2 M \delta\left(M^{2}-q^{2}\right)
$$


into (48). After performing the $\vec{p}_{4}$ integral using the first $\delta$-function, the $q^{0}$ integration using the second, and carrying out the trivial angular integration in the $\overrightarrow{p_{3}}$ integral, we arrive at

$$
\begin{aligned}
\frac{d^{2} \hat{\sigma}}{d M d \cos \theta}= & \frac{16 M}{3 \hat{s}} \frac{1}{(2 \pi)^{3}} \int \frac{d^{3} \vec{q}}{2 q^{0}} \int d E_{3}\left|\vec{p}_{3}\right| \delta\left(M^{2}-2 q \cdot p_{3}\right) \\
& \times \int d^{4} x e^{i\left(p_{1}+p_{2}-q\right) \cdot x} \operatorname{Tr}\left[\boldsymbol{H}\left(M, m_{t}, \cos \theta, \mu_{f}\right) \boldsymbol{W}\left(x, \mu_{f}\right)\right],
\end{aligned}
$$

where $\left|\vec{p}_{3}\right|=\sqrt{E_{3}^{2}-m_{t}^{2}}$ and $q_{0}=\sqrt{M^{2}+\vec{q}^{2}}$. In the partonic center-of-mass frame, we have $|\vec{q}|=\mathcal{O}(\sqrt{\hat{s}}(1-z))$, so we can set $q_{0}=M$ and drop $\vec{q}$ in the $\delta$-function. Then the integral over $\vec{q}$ produces a factor $\delta^{3}(\vec{x})$ from the exponential, and after a few manipulations we find

$$
\frac{d^{2} \hat{\sigma}}{d M d \cos \theta}=\frac{8 \pi \beta_{t}}{3 \hat{s} M} \operatorname{Tr}\left[\boldsymbol{H}\left(M, m_{t}, \cos \theta, \mu_{f}\right) \boldsymbol{S}\left(\sqrt{\hat{s}}(1-z), M, m_{t}, \cos \theta, \mu_{f}\right)\right],
$$

where the momentum-space soft function is defined by [64]

$$
\boldsymbol{S}\left(\sqrt{\hat{s}}(1-z), M, m_{t}, \cos \theta, \mu\right)=\sqrt{\hat{s}} \int \frac{d x_{0}}{4 \pi} e^{i \sqrt{\hat{s}}(1-z) x_{0} / 2} \boldsymbol{W}\left(x_{0}, \vec{x}=0, \mu\right) .
$$

The hadronic cross section (5) is now obtained by convoluting the partonic cross sections in (53) with the parton luminosities. Comparing with (10), we finally arrive at the factorized form of the hard-scattering kernel

$$
C\left(z, M, m_{t}, \cos \theta, \mu_{f}\right)=\operatorname{Tr}\left[\boldsymbol{H}\left(M, m_{t}, \cos \theta, \mu_{f}\right) \boldsymbol{S}\left(\sqrt{\hat{s}}(1-z), M, m_{t}, \cos \theta, \mu_{f}\right)\right],
$$

where we have set $\hat{s}=M^{2}$ everywhere except in the first argument of the soft function.

\section{The hard and soft functions at NLO}

In this section we describe the calculation of the soft and hard matrices up to NLO in perturbation theory.

\subsection{Hard functions}

The hard functions are related to products of Wilson coefficients, as shown in (49). To obtain the Wilson coefficients $C_{I m}$, one matches renormalized Green's functions in QCD with those in SCET. The matching can be done with any choice of external states and infrared (IR) regulators. It is by far simplest to use on-shell partonic states for $(q \bar{q}, g g) \rightarrow t \bar{t}$ scattering and dimensional regularization in $d=4-2 \epsilon$ dimensions to regularize both the ultraviolet (UV) and IR divergences. With this choice, the loop graphs in SCET are scaleless and vanish, so the effective-theory matrix elements are equal to their tree-level expressions multiplied by a UV renormalization matrix $\boldsymbol{Z}$. The matrix elements in QCD, on the other hand, are just the 
virtual corrections to the $(q \bar{q}, g g) \rightarrow t \bar{t}$ scattering amplitudes. The matching condition then reads $[55,74,79]$

$$
\lim _{\epsilon \rightarrow 0} \boldsymbol{Z}^{-1}\left(\epsilon, M, m_{t}, \cos \theta, \mu\right)\left|\mathcal{M}\left(\epsilon, M, m_{t}, \cos \theta\right)\right\rangle=\sum_{m}\left\langle\left\langle O_{m}\right\rangle\right\rangle_{\text {tree }}\left|C_{m}\left(M, m_{t}, \cos \theta, \mu\right)\right\rangle,
$$

where $\mathcal{M}$ is the UV-renormalized virtual QCD amplitude expressed in terms of $\alpha_{s}$ with $n_{l}=5$ active flavors. We have moved the SCET renormalization matrix $\boldsymbol{Z}$ to act on the QCD amplitude, so that both sides of the equation are finite in the limit $\epsilon \rightarrow 0$. The explicit results for the matrix elements $\boldsymbol{Z}_{I J}$ in our color basis for the $q \bar{q}$ and $g g$ channels can be found in [55].

In practice, we are not interested in the Wilson coefficients themselves, but rather the hard matrix $H_{I J}$. To calculate this, we first define

$$
\left|\mathcal{M}_{\text {ren }}\right\rangle \equiv \lim _{\epsilon \rightarrow 0} \boldsymbol{Z}^{-1}(\epsilon)|\mathcal{M}(\epsilon)\rangle=4 \pi \alpha_{s}\left[\left|\mathcal{M}_{\text {ren }}^{(0)}\right\rangle+\frac{\alpha_{s}}{4 \pi}\left|\mathcal{M}_{\text {ren }}^{(1)}\right\rangle+\ldots\right]
$$

and expand the hard function as

$$
\boldsymbol{H}=\alpha_{s}^{2} \frac{3}{8 d_{R}}\left(\boldsymbol{H}^{(0)}+\frac{\alpha_{s}}{4 \pi} \boldsymbol{H}^{(1)}+\ldots\right) .
$$

Using (57) and (58) to express the SCET matrix element in terms of the finite, IR-subtracted QCD amplitudes in the definition of the hard function (49), the matrix elements (50) can be written as

$$
\begin{aligned}
& H_{I J}^{(0)}=\frac{1}{4} \frac{1}{\left\langle c_{I} \mid c_{I}\right\rangle\left\langle c_{J} \mid c_{J}\right\rangle}\left\langle c_{I} \mid \mathcal{M}_{\mathrm{ren}}^{(0)}\right\rangle\left\langle\mathcal{M}_{\mathrm{ren}}^{(0)} \mid c_{J}\right\rangle, \\
& H_{I J}^{(1)}=\frac{1}{4} \frac{1}{\left\langle c_{I} \mid c_{I}\right\rangle\left\langle c_{J} \mid c_{J}\right\rangle}\left[\left\langle c_{I} \mid \mathcal{M}_{\mathrm{ren}}^{(0)}\right\rangle\left\langle\mathcal{M}_{\mathrm{ren}}^{(1)} \mid c_{J}\right\rangle+\left\langle c_{I} \mid \mathcal{M}_{\mathrm{ren}}^{(1)}\right\rangle\left\langle\mathcal{M}_{\mathrm{ren}}^{(0)} \mid c_{J}\right\rangle\right] .
\end{aligned}
$$

The leading-order result for the $q \bar{q}$ channel follows from a simple calculation and reads

$$
\boldsymbol{H}_{q \bar{q}}^{(0)}=\left(\begin{array}{ll}
0 & 0 \\
0 & 2
\end{array}\right)\left[\frac{t_{1}^{2}+u_{1}^{2}}{M^{4}}+\frac{2 m_{t}^{2}}{M^{2}}\right],
$$

while that for the $g g$ channel is

$$
\boldsymbol{H}_{g g}^{(0)}=\left(\begin{array}{ccc}
\frac{1}{N^{2}} & \frac{1}{N} \frac{t_{1}-u_{1}}{M^{2}} & \frac{1}{N} \\
\frac{1}{N} \frac{t_{1}-u_{1}}{M^{2}} & \frac{\left(t_{1}-u_{1}\right)^{2}}{M^{4}} & \frac{t_{1}-u_{1}}{M^{2}} \\
\frac{1}{N} & \frac{t_{1}-u_{1}}{M^{2}} & 1
\end{array}\right) \frac{M^{4}}{2 t_{1} u_{1}}\left[\frac{t_{1}^{2}+u_{1}^{2}}{M^{4}}+\frac{4 m_{t}^{2}}{M^{2}}-\frac{4 m_{t}^{4}}{t_{1} u_{1}}\right] .
$$

To calculate the NLO hard function requires the one-loop virtual corrections to the partonic scattering amplitudes, decomposed into the singlet-octet basis. Although results for the NLO virtual corrections interfered with the Born-level amplitudes exist in the literature [13-15], results for the one-loop amplitude decomposed into our color basis are not available and must be calculated from scratch. For this purpose we use in-house routines written in the computer 
algebraic system FORM [80]. The results are rather lengthy (especially for the $g g$ channel) and are in a Mathematica package which can be obtained from the authors upon request.

We have been able to perform several checks on our results. First, we have verified that applying the renormalization factor $\boldsymbol{Z}$ to the tree-level amplitude indeed absorbs the IR poles in the UV-renormalized QCD amplitudes at one-loop order. Second, we have checked that inserting the results for the products of one-loop hard functions and tree-level soft functions, given in (65) below, into the formula for the differential cross section, we reproduce the results of [13-15], as required. Finally, using the one-loop hard functions, we were able to calculate the IR singularities of the two-loop amplitudes in [55], which agree with all the available results in the literature [22-24].

\subsection{Soft functions}

The soft functions are given by the vacuum expectation values of the soft Wilson-loop operators, as defined in (49). In what follows we will calculate the one-loop corrections to these objects directly in position space. When performing the resummation in the next section, it will be more convenient to work with the Laplace-transformed functions. They are defined as

$$
\begin{aligned}
\tilde{\boldsymbol{s}}\left(L, M, m_{t}, \cos \theta, \mu\right) & =\frac{1}{\sqrt{\hat{s}}} \int_{0}^{\infty} d \omega \exp \left(-\frac{\omega}{e^{\gamma_{E}} \mu e^{L / 2}}\right) \boldsymbol{S}\left(\omega, M, m_{t}, \cos \theta, \mu\right) \\
& =\boldsymbol{W}\left(x_{0}=\frac{-2 i}{e^{\gamma_{E}} \mu e^{L / 2}}, \mu\right)
\end{aligned}
$$

where the second equality was shown in [64] and follows from the functional form of positionspace Wilson loops [81].

We expand the soft functions in power of $\alpha_{s}$ as

$$
\tilde{\boldsymbol{s}}=\tilde{\boldsymbol{s}}^{(0)}+\frac{\alpha_{s}}{4 \pi} \tilde{\boldsymbol{s}}^{(1)}+\left(\frac{\alpha_{s}}{4 \pi}\right)^{2} \tilde{\boldsymbol{s}}^{(2)}+\ldots
$$

At leading order, the Wilson loop is just the unit matrix, so $\tilde{s}_{I J}^{(0)}=\left\langle c_{I} \mid c_{J}\right\rangle / d_{R}$, and it is easy to show that

$$
\tilde{\boldsymbol{s}}_{q \bar{q}}^{(0)}=\left(\begin{array}{cc}
N & 0 \\
0 & \frac{C_{F}}{2}
\end{array}\right), \quad \tilde{\boldsymbol{s}}_{g g}^{(0)}=\left(\begin{array}{ccc}
N & 0 & 0 \\
0 & \frac{N}{2} & 0 \\
0 & 0 & \frac{N^{2}-4}{2 N}
\end{array}\right) .
$$

At NLO, the soft functions receive contributions from the diagrams depicted in Figure 1. The calculation is similar to that in [81]. To evaluate the diagrams we associate an eikonal factor $v_{i}^{\mu} / k \cdot v_{i}$ multiplied by a color generator $\boldsymbol{T}_{i}$ for each attachment of a gluon to a particle with velocity $v_{i}$ (we define $v_{1}=n$ and $v_{2}=\bar{n}$ ), and contract with the cut gluon propagator in position space, which in Feynman gauge reads

$$
D_{+}^{\mu \nu}(x)=-g^{\mu \nu} \int \frac{d^{d} k}{(2 \pi)^{d}} e^{-i k \cdot x}(2 \pi) \delta\left(k^{2}\right) \theta\left(k^{0}\right) .
$$




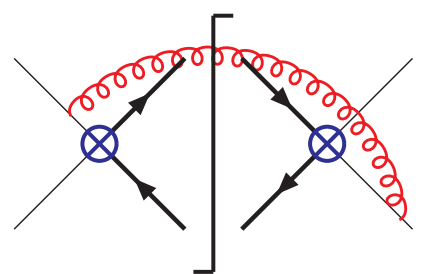

$\mathcal{I}_{12}$

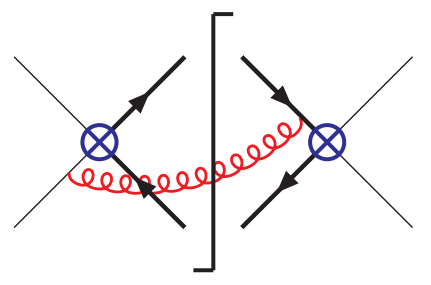

$\mathcal{I}_{23}$

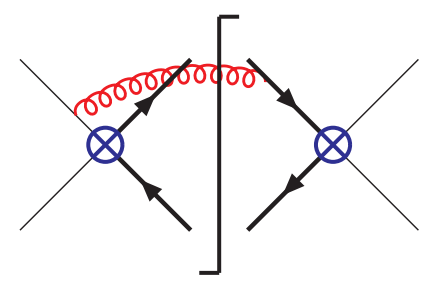

$\mathcal{I}_{13}$

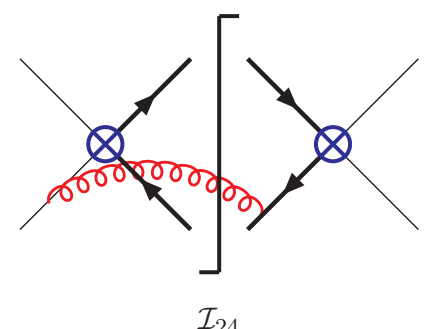

$\mathcal{I}_{24}$

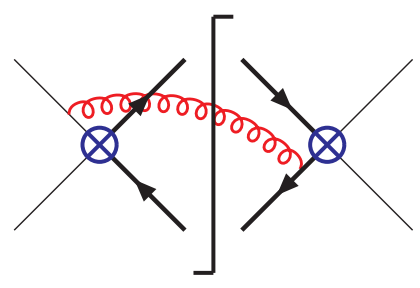

$\mathcal{I}_{14}$

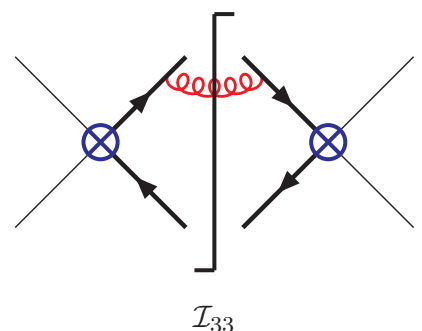

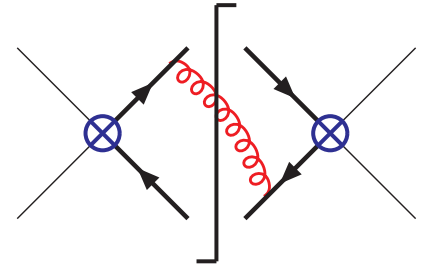

$\mathcal{I}_{34}$

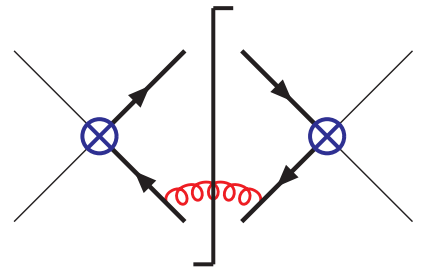

$\mathcal{I}_{44}$

Figure 1: Diagrams contributing to the soft functions at NLO. The thick lines represent Wilson lines in the time-like directions $v_{3}$ and $v_{4}$, the thin lines Wilson lines in the light-like directions $n$ and $\bar{n}$, and the cut curly lines represent the cut gluon propagator (66).

We can then write the bare soft function in position space as

$$
\boldsymbol{W}_{\text {bare }}^{(1)}\left(\epsilon, x_{0}, \mu\right)=\sum_{i, j} \boldsymbol{w}_{i j} \mathcal{I}_{i j}\left(\epsilon, x_{0}, \mu\right)
$$

where the matrices $\boldsymbol{w}_{i j}$ are related to products of color generators and will be given in (72) and (73) below. The integrals $\mathcal{I}_{i j}$ are defined as

$$
\mathcal{I}_{i j}\left(\epsilon, x_{0}, \mu\right)=-\frac{\left(4 \pi \mu^{2}\right)^{\epsilon}}{\pi^{2-\epsilon}} v_{i} \cdot v_{j} \int d^{d} k \frac{e^{-i k^{0} x_{0}}}{v_{i} \cdot k v_{j} \cdot k}(2 \pi) \delta\left(k^{2}\right) \theta\left(k^{0}\right),
$$

which are obviously symmetric in the indices $i$ and $j$. The integrals $\mathcal{I}_{11}=\mathcal{I}_{22}=0$, and the non-vanishing integrals are

$$
\begin{gathered}
\mathcal{I}_{12}=-\left(\frac{2}{\epsilon^{2}}+\frac{2}{\epsilon} L_{0}+L_{0}^{2}+\frac{\pi^{2}}{6}\right), \\
\mathcal{I}_{33}=\mathcal{I}_{44}=\frac{2}{\epsilon}+2 L_{0}-\frac{2}{\beta_{t}} \ln x_{s},
\end{gathered}
$$




$$
\begin{aligned}
\mathcal{I}_{34} & =-\frac{1+x_{s}^{2}}{1-x_{s}^{2}}\left[\left(\frac{2}{\epsilon}+2 L_{0}\right) \ln x_{s}-\ln ^{2} x_{s}+4 \ln x_{s} \ln \left(1-x_{s}\right)+4 \operatorname{Li}_{2}\left(x_{s}\right)-\frac{2 \pi^{2}}{3}\right], \\
\mathcal{I}_{13}=\mathcal{I}_{24} & =-\left[\frac{1}{2}\left(L_{0}-\ln \frac{\left(1+y_{t}\right)^{2} x_{s}}{\left(1+x_{s}\right)^{2}}\right)^{2}+\frac{\pi^{2}}{12}+2 \operatorname{Li}_{2}\left(\frac{1-x_{s} y_{t}}{1+x_{s}}\right)+2 \operatorname{Li}_{2}\left(\frac{x_{s}-y_{t}}{1+x_{s}}\right)\right], \\
\mathcal{I}_{14}=\mathcal{I}_{23} & =\mathcal{I}_{13}\left(y_{t} \rightarrow z_{u}\right),
\end{aligned}
$$

where $x_{s}=\left(1-\beta_{t}\right) /\left(1+\beta_{t}\right), y_{t}=-t_{1} / m_{t}^{2}-1, z_{u}=-u_{1} / m_{t}^{2}-1$, and

$$
L_{0}=\ln \left(-\frac{\mu^{2} x_{0}^{2} e^{2 \gamma_{E}}}{4}\right) \text {. }
$$

The renormalized soft functions $\boldsymbol{W}^{(1)}$ can then be obtained by subtracting the divergent part from $\boldsymbol{W}_{\text {bare }}^{(1)}$. Later on we will need the Laplace-transformed function $\tilde{\boldsymbol{s}}$, which according to (63) is obtained by replacing $L_{0} \rightarrow-L$. To finish the calculation, we must also determine the matrix elements of

$$
\left(\boldsymbol{w}_{i j}\right)_{I J}=\frac{1}{d_{R}}\left\langle c_{I}\left|\boldsymbol{T}_{i} \cdot \boldsymbol{T}_{j}\right| c_{J}\right\rangle
$$

For the $q \bar{q}$ channel, the results are

$$
\begin{aligned}
& \boldsymbol{w}_{12}^{q \bar{q}}=\boldsymbol{w}_{34}^{q \bar{q}}=-\frac{C_{F}}{4 N}\left(\begin{array}{cc}
4 N^{2} & 0 \\
0 & -1
\end{array}\right), \\
& \boldsymbol{w}_{33}^{q \bar{q}}=\boldsymbol{w}_{44}^{q \bar{q}}=\frac{C_{F}}{2}\left(\begin{array}{cc}
2 N & 0 \\
0 & C_{F}
\end{array}\right), \\
& \boldsymbol{w}_{13}^{q \bar{q}}=\boldsymbol{w}_{24}^{q \bar{q}}=-\frac{C_{F}}{2}\left(\begin{array}{cc}
0 & 1 \\
1 & 2 C_{F}-\frac{N}{2}
\end{array}\right), \\
& \boldsymbol{w}_{14}^{q \bar{q}}=\boldsymbol{w}_{23}^{q \bar{q}}=-\frac{C_{F}}{2 N}\left(\begin{array}{cc}
0 & -N \\
-N & 1
\end{array}\right),
\end{aligned}
$$

while for the $g g$ channel we obtain

$$
\begin{aligned}
& \boldsymbol{w}_{12}^{g g}=-\frac{1}{4}\left(\begin{array}{ccc}
4 N^{2} & 0 & 0 \\
0 & N^{2} & 0 \\
0 & 0 & N^{2}-4
\end{array}\right), \\
& \boldsymbol{w}_{34}^{g g}=-\left(\begin{array}{ccc}
C_{F} N & 0 & 0 \\
0 & -\frac{1}{4} & 0 \\
0 & 0 & -\frac{N^{2}-4}{4 N^{2}}
\end{array}\right),
\end{aligned}
$$




$$
\begin{aligned}
& \boldsymbol{w}_{33}^{g g}=\boldsymbol{w}_{44}^{g g}=\frac{C_{F}}{2 N}\left(\begin{array}{ccc}
2 N^{2} & 0 & 0 \\
0 & N^{2} & 0 \\
0 & 0 & N^{2}-4
\end{array}\right), \\
& \boldsymbol{w}_{13}^{g g}=\boldsymbol{w}_{24}^{g g}=-\frac{1}{8}\left(\begin{array}{ccc}
0 & 4 N & 0 \\
4 N & N^{2} & N^{2}-4 \\
0 & N^{2}-4 & N^{2}-4
\end{array}\right), \\
& \boldsymbol{w}_{14}^{g g}=\boldsymbol{w}_{23}^{g g}=-\frac{1}{8}\left(\begin{array}{ccc}
0 & -4 N & 0 \\
-4 N & N^{2} & -\left(N^{2}-4\right) \\
0 & -\left(N^{2}-4\right) & N^{2}-4
\end{array}\right) .
\end{aligned}
$$

The NLO contributions to the cross section from real emissions in the soft limit have been known for some time [18]. Transforming our results to momentum space, we have checked that the integrals (68) are consistent with those given in Appendix A of that paper (after taking into account some misprints in [18] later corrected in [82]). As another check, we have used our results along with the one-loop hard functions from the previous section to calculate the total partonic cross sections at NLO in the limit $\beta \rightarrow 0$ (see Section 6.3), reproducing the analytic expressions from [16]. Finally, using the RG invariance of the cross section we will derive the RG equation for $\tilde{\boldsymbol{s}}$ in the next section. We have checked that our one-loop result satisfies this equation, which also justifies our procedure of simply subtracting the $1 / \epsilon$ poles in the bare function to get the renormalized results.

\section{Threshold resummation in SCET}

In the region where the cross section is dominated by the threshold terms, one needs to resum the leading singular terms in $(1-z)$ to all orders in perturbation theory. This is accomplished by deriving and solving RG equations for the hard and soft functions in the effective theory, which will be described in what follows. Since these equations contain information on the logarithmic structure of the hard-scattering kernels at higher-orders in perturbation theory, they can also be used to derive an approximate NNLO formula for the differential cross section in the threshold region. We discuss this further in Section 5.2.

\subsection{RG evolution and resummation at NNLL}

The hard function satisfies the evolution equation

$$
\begin{aligned}
\frac{d}{d \ln \mu} \boldsymbol{H}\left(M, m_{t}, \cos \theta, \mu\right)= & \boldsymbol{\Gamma}_{H}\left(M, m_{t}, \cos \theta, \mu\right) \boldsymbol{H}\left(M, m_{t}, \cos \theta, \mu\right) \\
& +\boldsymbol{H}\left(M, m_{t}, \cos \theta, \mu\right) \boldsymbol{\Gamma}_{H}^{\dagger}\left(M, m_{t}, \cos \theta, \mu\right) .
\end{aligned}
$$

Using (50), we can write the above equation in a matrix form, where the matrix elements of $\boldsymbol{\Gamma}_{H}$ are defined according to (38), and can be obtained from the matrices $\boldsymbol{\Gamma}_{q \bar{q}}$ or $\boldsymbol{\Gamma}_{g g}$ in [55]. 
The form of the evolution equation follows from (58) and (60), along with the defining relation

$$
\boldsymbol{Z}^{-1} \frac{d}{d \ln \mu} \boldsymbol{Z}=-\boldsymbol{\Gamma}_{H}
$$

for the anomalous dimension. The explicit results to two-loop order are

$$
\begin{aligned}
\boldsymbol{\Gamma}_{q \bar{q}}= & {\left[C_{F} \gamma_{\text {cusp }}\left(\alpha_{s}\right)\left(\ln \frac{M^{2}}{\mu^{2}}-i \pi\right)+C_{F} \gamma_{\text {cusp }}\left(\beta_{34}, \alpha_{s}\right)+2 \gamma^{q}\left(\alpha_{s}\right)+2 \gamma^{Q}\left(\alpha_{s}\right)\right] \mathbf{1} } \\
& +\frac{N}{2}\left[\gamma_{\text {cusp }}\left(\alpha_{s}\right)\left(\ln \frac{t_{1}^{2}}{M^{2} m_{t}^{2}}+i \pi\right)-\gamma_{\text {cusp }}\left(\beta_{34}, \alpha_{s}\right)\right]\left(\begin{array}{ll}
0 & 0 \\
0 & 1
\end{array}\right) \\
& +\gamma_{\text {cusp }}\left(\alpha_{s}\right) \ln \frac{t_{1}^{2}}{u_{1}^{2}}\left[\left(\begin{array}{cc}
0 & \frac{C_{F}}{2 N} \\
1 & -\frac{1}{N}
\end{array}\right)+\frac{\alpha_{s}}{4 \pi} g\left(\beta_{34}\right)\left(\begin{array}{cc}
0 & \frac{C_{F}}{2} \\
-N & 0
\end{array}\right)\right]
\end{aligned}
$$

and

$$
\begin{aligned}
\boldsymbol{\Gamma}_{g g}= & {\left[N \gamma_{\text {cusp }}\left(\alpha_{s}\right)\left(\ln \frac{M^{2}}{\mu^{2}}-i \pi\right)+C_{F} \gamma_{\text {cusp }}\left(\beta_{34}, \alpha_{s}\right)+2 \gamma^{g}\left(\alpha_{s}\right)+2 \gamma^{Q}\left(\alpha_{s}\right)\right] \mathbf{1} } \\
& +\frac{N}{2}\left[\gamma_{\text {cusp }}\left(\alpha_{s}\right)\left(\ln \frac{t_{1}^{2}}{M^{2} m_{t}^{2}}+i \pi\right)-\gamma_{\text {cusp }}\left(\beta_{34}, \alpha_{s}\right)\right]\left(\begin{array}{lll}
0 & 0 & 0 \\
0 & 1 & 0 \\
0 & 0 & 1
\end{array}\right) \\
& +\gamma_{\text {cusp }}\left(\alpha_{s}\right) \ln \frac{t_{1}^{2}}{u_{1}^{2}}\left[\left(\begin{array}{ccc}
0 & \frac{1}{2} & 0 \\
1 & -\frac{N}{4} & \frac{N^{2}-4}{4 N} \\
0 & \frac{N}{4} & -\frac{N}{4}
\end{array}\right)+\frac{\alpha_{s}}{4 \pi} g\left(\beta_{34}\right)\left(\begin{array}{ccc}
0 & \frac{N}{2} & 0 \\
-N & 0 & 0 \\
0 & 0 & 0
\end{array}\right)\right],
\end{aligned}
$$

where the various anomalous-dimension functions can be found in the Appendix, and the cusp angle $\beta_{34}=i \pi-\ln \left(1+\beta_{t}\right) /\left(1-\beta_{t}\right)$. The solution to the evolution equation can be written as

$$
\boldsymbol{H}\left(M, m_{t}, \cos \theta, \mu\right)=\boldsymbol{U}\left(M, m_{t}, \cos \theta, \mu_{h}, \mu\right) \boldsymbol{H}\left(M, m_{t}, \cos \theta, \mu_{h}\right) \boldsymbol{U}^{\dagger}\left(M, m_{t}, \cos \theta, \mu_{h}, \mu\right),
$$

where the unitary matrix $\boldsymbol{U}$ satisfies the equation

$$
\frac{d}{d \ln \mu} \boldsymbol{U}\left(M, m_{t}, \cos \theta, \mu_{h}, \mu\right)=\boldsymbol{\Gamma}_{H}\left(M, m_{t}, \cos \theta, \mu\right) \boldsymbol{U}\left(M, m_{t}, \cos \theta, \mu_{h}, \mu\right) .
$$

The matching scale $\mu_{h}$ must be chosen of order a typical hard scale, so that the matching condition for the hard function is free of large logarithms. With the help of the evolution matrix $\boldsymbol{U}$, the hard function can then be evolved to an arbitrary scale $\mu$. The formal solution to this equation is

$$
\boldsymbol{U}\left(M, m_{t}, \cos \theta, \mu_{h}, \mu\right)=\mathcal{P} \exp \int_{\mu_{h}}^{\mu} \frac{d \mu^{\prime}}{\mu^{\prime}} \boldsymbol{\Gamma}_{H}\left(M, m_{t}, \cos \theta, \mu^{\prime}\right),
$$


where the path-ordering is necessary because $\boldsymbol{\Gamma}_{H}$ is a matrix. To evaluate the path-ordered exponential, it is convenient to separate the explicit logarithmic dependence on the scale $\mu$, which is related to Sudakov double logarithms, from the remaining piece, which is related to single logarithmic evolution. We thus write the anomalous dimension as

$$
\Gamma_{H}\left(M, m_{t}, \cos \theta, \mu\right)=\Gamma_{\text {cusp }}\left(\alpha_{s}\right)\left(\ln \frac{M^{2}}{\mu^{2}}-i \pi\right) \mathbf{1}+\gamma^{h}\left(M, m_{t}, \cos \theta, \alpha_{s}\right),
$$

where $\Gamma_{\text {cusp }}$ is equal to $C_{F} \gamma_{\text {cusp }}$ for $q \bar{q}$ and $N \gamma_{\text {cusp }}$ for $g g$, and the matrices $\gamma^{h}$ are defined through a comparison with (76) and (77). Since the term proportional to $\Gamma_{\text {cusp }}$ multiplies the unit matrix, we can factor this piece out of the path-ordering and evaluate it using standard techniques. The result for the evolution matrix is then

$$
\boldsymbol{U}\left(M, m_{t}, \cos \theta, \mu_{h}, \mu\right)=\exp \left[2 S\left(\mu_{h}, \mu\right)-a_{\Gamma}\left(\mu_{h}, \mu\right)\left(\ln \frac{M^{2}}{\mu_{h}^{2}}-i \pi\right)\right] \boldsymbol{u}\left(M, m_{t}, \cos \theta, \mu_{h}, \mu\right) .
$$

The RG exponents in the square brackets of the exponential factor are given by

$$
S\left(\mu_{h}, \mu\right)=-\int_{\alpha_{s}\left(\mu_{h}\right)}^{\alpha_{s}(\mu)} d \alpha \frac{\Gamma_{\text {cusp }}(\alpha)}{\beta(\alpha)} \int_{\alpha_{s}\left(\mu_{h}\right)}^{\alpha} \frac{d \alpha^{\prime}}{\beta\left(\alpha^{\prime}\right)}, \quad a_{\Gamma}\left(\mu_{h}, \mu\right)=-\int_{\alpha_{s}\left(\mu_{h}\right)}^{\alpha_{s}(\mu)} d \alpha \frac{\Gamma_{\text {cusp }}(\alpha)}{\beta(\alpha)},
$$

where $\beta\left(\alpha_{s}\right)=d \alpha_{s} / d \ln \mu$ is the QCD $\beta$-function. The quantity $\boldsymbol{u}$ contains the non-trivial matrix evolution due to $\gamma^{h}$ and reads

$$
\boldsymbol{u}\left(M, m_{t}, \cos \theta, \mu_{h}, \mu\right)=\mathcal{P} \exp \int_{\alpha_{s}\left(\mu_{h}\right)}^{\alpha_{s}(\mu)} \frac{d \alpha}{\beta(\alpha)} \gamma^{h}\left(M, m_{t}, \cos \theta, \alpha\right)
$$

The perturbative solutions to the above equations are reviewed in the Appendix.

We now turn to the evolution of the soft function. We derive its evolution equation by using the RG invariance of the cross section,

$$
\frac{d}{d \ln \mu} \operatorname{Tr}[\boldsymbol{H} \boldsymbol{S}] \otimes f f=0,
$$

along with the evolution equations for the hard function and parton luminosities. The evolution equation for the hard function was given above, and the parton luminosity functions satisfy the DGLAP equations [83-85]. While the full DGLAP equations involve flavor mixing, what we need here is the $x \rightarrow 1$ limit of them, which is flavor-diagonal and can be written as

$$
\frac{d}{d \ln \mu} f f(y, \mu)=2 \int_{y}^{1} \frac{d x}{x} P(x) f f(y / x, \mu),
$$

where $P(x)$ is given by

$$
P(x)=\frac{2 \Gamma_{\text {cusp }}\left(\alpha_{s}\right)}{(1-x)_{+}}+2 \gamma^{\phi}\left(\alpha_{s}\right) \delta(1-x) .
$$


The evolution for the momentum-space soft function is then

$$
\begin{aligned}
\frac{d}{d \ln \mu} \boldsymbol{S}\left(\omega, M, m_{t},\right. & \cos \theta, \mu)=-\left[2 \Gamma_{\text {cusp }}\left(\alpha_{s}\right) \ln \frac{\omega}{\mu}+\gamma^{s \dagger}\left(M, m_{t}, \cos \theta, \alpha_{s}\right)\right] \boldsymbol{S}\left(\omega, M, m_{t}, \cos \theta, \mu\right) \\
& -\boldsymbol{S}\left(\omega, M, m_{t}, \cos \theta, \mu\right)\left[2 \Gamma_{\text {cusp }}\left(\alpha_{s}\right) \ln \frac{\omega}{\mu}+\boldsymbol{\gamma}^{s}\left(M, m_{t}, \cos \theta, \alpha_{s}\right)\right] \\
& -4 \Gamma_{\text {cusp }}\left(\alpha_{s}\right) \int_{0}^{\omega} d \omega^{\prime} \frac{\boldsymbol{S}\left(\omega^{\prime}, M, m_{t}, \cos \theta, \mu\right)-\boldsymbol{S}\left(\omega, M, m_{t}, \cos \theta, \mu\right)}{\omega-\omega^{\prime}}
\end{aligned}
$$

where we have defined

$$
\gamma^{s}\left(M, m_{t}, \cos \theta, \alpha_{s}\right)=\gamma^{h}\left(M, m_{t}, \cos \theta, \alpha_{s}\right)+2 \gamma^{\phi}\left(\alpha_{s}\right) \mathbf{1}
$$

As in [64], the non-local evolution equation for the soft function can be turned into a local one by the Laplace transformation (63). The evolution equation for the Laplace-transformed function reads

$$
\begin{aligned}
\frac{d}{d \ln \mu} \tilde{\boldsymbol{s}} & \left(\ln \frac{M^{2}}{\mu^{2}}, M, m_{t}, \cos \theta, \mu\right)= \\
& -\left[\Gamma_{\text {cusp }}\left(\alpha_{s}\right) \ln \frac{M^{2}}{\mu^{2}}+\gamma^{s \dagger}\left(M, m_{t}, \cos \theta, \alpha_{s}\right)\right] \tilde{\boldsymbol{s}}\left(\ln \frac{M^{2}}{\mu^{2}}, M, m_{t}, \cos \theta, \mu\right) \\
& -\tilde{\boldsymbol{s}}\left(\ln \frac{M^{2}}{\mu^{2}}, M, m_{t}, \cos \theta, \mu\right)\left[\Gamma_{\text {cusp }}\left(\alpha_{s}\right) \ln \frac{M^{2}}{\mu^{2}}+\gamma^{s}\left(M, m_{t}, \cos \theta, \alpha_{s}\right)\right] .
\end{aligned}
$$

This can be solved using the same methods as for the hard function. Transforming the results back to momentum space, we find

$$
\begin{aligned}
& \boldsymbol{S}\left(\omega, M, m_{t}, \cos \theta, \mu_{f}\right)=\sqrt{\hat{s}} \exp \left[-4 S\left(\mu_{s}, \mu_{f}\right)+4 a_{\gamma^{\phi}}\left(\mu_{s}, \mu_{f}\right)\right] \\
& \times \boldsymbol{u}^{\dagger}\left(M, m_{t}, \cos \theta, \mu_{f}, \mu_{s}\right) \tilde{\boldsymbol{s}}\left(\partial_{\eta}, M, m_{t}, \cos \theta, \mu_{s}\right) \boldsymbol{u}\left(M, m_{t}, \cos \theta, \mu_{f}, \mu_{s}\right) \frac{1}{\omega}\left(\frac{\omega}{\mu_{s}}\right)^{2 \eta} \frac{e^{-2 \gamma_{E} \eta}}{\Gamma(2 \eta)},
\end{aligned}
$$

where $\eta=2 a_{\Gamma}\left(\mu_{s}, \mu_{f}\right)$. The soft scale $\mu_{s}$ should be chosen such that the contribution from the soft function to the cross section is perturbatively well-behaved, and will be discussed in detail in Section 6.

Combining the results for the hard and soft functions, our final resummed expression for the hard-scattering kernel is

$$
\begin{aligned}
C\left(z, M, m_{t}, \cos \theta, \mu_{f}\right) & =\exp \left[4 a_{\gamma^{\phi}}\left(\mu_{s}, \mu_{f}\right)\right] \\
\times \operatorname{Tr} & {\left[\boldsymbol{U}\left(M, m_{t}, \cos \theta, \mu_{h}, \mu_{s}\right) \boldsymbol{H}\left(M, m_{t}, \cos \theta, \mu_{h}\right) \boldsymbol{U}^{\dagger}\left(M, m_{t}, \cos \theta, \mu_{h}, \mu_{s}\right)\right.}
\end{aligned}
$$




$$
\left.\times \tilde{\boldsymbol{s}}\left(\ln \frac{M^{2}}{\mu_{s}^{2}}+\partial_{\eta}, M, m_{t}, \cos \theta, \mu_{s}\right)\right] \frac{e^{-2 \gamma_{E} \eta}}{\Gamma(2 \eta)} \frac{z^{-\eta}}{(1-z)^{1-2 \eta}} .
$$

For values $\mu_{s}<\mu_{f}$ the parameter $\eta<0$, and one must use a subtraction at $z=1$ and analytic continuation to express integrals over $z$ in terms of star (or plus) distributions [86]. Formula (92) can be evaluated order-by-order in RG-improved perturbation theory, using the standard counting $\ln \mu_{h} / \mu_{s} \sim \ln (1-z) \sim 1 / \alpha_{s}$. The perturbative solutions for the RG factors needed to evaluate the evolution matrix $\boldsymbol{U}$ to NLO in this counting scheme are given in (A.2), (A.3), and (A.5) of the Appendix. The correspondence between this counting and the standard counting of logarithms (e.g. NLL, NNLL), along with the accuracy of the anomalous dimensions and matching functions needed at a given order, can be summarized as follows:

\begin{tabular}{|c|c|c|c|c|}
\hline RG-improved PT & log accuracy & $\Gamma_{\text {cusp }}$ & $\boldsymbol{\gamma}^{h}, \gamma^{\phi}$ & $\boldsymbol{H}, \tilde{\boldsymbol{s}}$ \\
\hline LO & NLL & 2-loop & 1-loop & tree-level \\
\hline NLO & NNLL & 3-loop & 2-loop & 1-loop \\
\hline
\end{tabular}

In the remainder of the paper we will use the logarithmic counting (e.g. NNLL) when referring to the resummed results obtained in this section. These results are valid for the leadingorder term in the threshold expansion in $(1-z)$, whereas the full result at NLO in fixedorder perturbation theory also contains information on subleading terms. In phenomenological applications we can match the resummed results with the NLO fixed-order results to achieve an NLO+NNLL precision. The method for doing this is described in Section 6 .

\subsection{Approximate NNLO results}

In the previous subsection we derived a formula for the resummed differential cross section, which is valid up to NNLL order. Starting from (92), it is also possible to obtain expressions for the differential cross section which are valid in fixed-order perturbation theory [56]. Indeed, our results allow one to obtain analytic expression for all of the coefficients multiplying singular plus distributions in the variable $(1-z)$ appearing in the hard-scattering kernels up to NNLO. With the same method, which is outlined below, it is also possible to determine analytically, up to $\mathcal{O}\left(\alpha_{s}^{4}\right)$, the scale-dependent parts of the coefficient multiplying $\delta(1-z)$.

In order to derive fixed-order formulas from (92), we first set $\mu_{h}=\mu_{s}=\mu_{f}=\mu$. In that case the evolution matrix $\boldsymbol{U}$ is equal to unity, and $\eta=2 a_{\Gamma}\left(\mu_{f}, \mu_{s}\right) \rightarrow 0$. The formula for the hard-scattering kernels then becomes

$$
C\left(z, M, m_{t}, \cos \theta, \mu\right)=\left.\tilde{c}\left(\partial_{\eta}, M, m_{t}, \cos \theta, \mu\right)\left(\frac{M}{\mu}\right)^{2 \eta} \frac{e^{-2 \gamma_{E} \eta}}{\Gamma(2 \eta)} \frac{z^{-\eta}}{(1-z)^{1-2 \eta}}\right|_{\eta=0}
$$

where

$$
\tilde{c}\left(L, M, m_{t}, \cos \theta, \mu\right)=\operatorname{Tr}\left[\boldsymbol{H}\left(M, m_{t}, \cos \theta, \mu\right) \tilde{\boldsymbol{s}}\left(L, M, m_{t}, \cos \theta, \mu\right)\right]
$$


By using (74) and (90) in combination with the analytic expressions for the hard and soft functions at NLO, it is possible to determine all terms proportional to $\ln \mu$ in the two-loop hard function $\boldsymbol{H}^{(2)}\left(M, m_{t}, \cos \theta, \mu\right)$, as well as all terms proportional to $L$ in the two-loop soft function $\tilde{\boldsymbol{s}}^{(2)}\left(L, M, m_{t}, \cos \theta, \mu\right)$. This information allows us to derive an approximate expression for $\tilde{c}$ at NNLO. By inserting that formula for $\tilde{c}$ into (93), we obtain the corresponding NNLO expression for the hard-scattering kernel $C$. The results are conventionally written in terms of the plus distributions

$$
P_{n}(z)=\left[\frac{\ln ^{n}(1-z)}{1-z}\right]_{+} .
$$

However, the right-hand side of (93) is more conveniently expressed in terms of the distributions

$$
P_{n}^{\prime}(z)=\left[\frac{1}{1-z} \ln ^{n}\left(\frac{M^{2}(1-z)^{2}}{\mu^{2} z}\right)\right]_{+} .
$$

It is possible to show that taking the derivatives with respect to $\eta$ and the limit $\eta \rightarrow 0$ in (93) is equivalent to making the following set of replacements in $\tilde{c}\left(L, M, m_{t}, \cos \theta, \mu\right)$ :

$$
\begin{aligned}
1 & \rightarrow \delta(1-z) \\
L & \rightarrow 2 P_{0}^{\prime}(z)+\delta(1-z) \ln \left(\frac{M^{2}}{\mu^{2}}\right) \\
L^{2} & \rightarrow 4 P_{1}^{\prime}(z)+\delta(1-z) \ln ^{2}\left(\frac{M^{2}}{\mu^{2}}\right) \\
L^{3} & \rightarrow 6 P_{2}^{\prime}(z)-4 \pi^{2} P_{0}^{\prime}(z)+\delta(1-z)\left[\ln ^{3}\left(\frac{M^{2}}{\mu^{2}}\right)+4 \zeta_{3}\right] \\
L^{4} & \rightarrow 8 P_{3}^{\prime}(z)-16 \pi^{2} P_{1}^{\prime}(z)+128 \zeta_{3} P_{0}^{\prime}(z)+\delta(1-z)\left[\ln ^{4}\left(\frac{M^{2}}{\mu^{2}}\right)+16 \zeta_{3} \ln \left(\frac{M^{2}}{\mu^{2}}\right)\right] .
\end{aligned}
$$

In order to translate the $P_{n}^{\prime}$ into the conventional $P_{n}$ distributions, we employ the general relation

$$
\begin{aligned}
P_{n}^{\prime}(z)= & \sum_{k=0}^{n}\left(\begin{array}{l}
n \\
k
\end{array}\right) \ln ^{n-k}\left(\frac{M^{2}}{\mu^{2}}\right)\left[2^{k} P_{k}(z)\right. \\
& \left.+\sum_{j=0}^{k-1}\left(\begin{array}{l}
k \\
j
\end{array}\right) 2^{j}(-1)^{k-j}\left(\frac{\ln ^{j}(1-z) \ln ^{k-j} z}{1-z}-\delta(1-z) \int_{0}^{1} d x \frac{\ln ^{j}(1-x) \ln ^{k-j} x}{1-x}\right)\right] .
\end{aligned}
$$

The final result for the hard-scattering kernels at NNLO can be written as

$$
C^{(2)}\left(z, M, m_{t}, \cos \theta, \mu\right)=D_{3}\left[\frac{\ln ^{3}(1-z)}{1-z}\right]_{+}+D_{2}\left[\frac{\ln ^{2}(1-z)}{1-z}\right]_{+}
$$




$$
+D_{1}\left[\frac{\ln (1-z)}{1-z}\right]_{+}+D_{0}\left[\frac{1}{1-z}\right]_{+}+C_{0} \delta(1-z)+R(z) .
$$

The coefficients $D_{0}, \ldots, D_{3}$ and $C_{0}$ are functions of the variables $M, m_{t}, \cos \theta$, and $\mu$. The analytic expression for $D_{i}(i=1,2,3)$ were first derived in [52] starting from resummed formulas in Mellin moment space. In [56] the coefficients $D_{0}, \ldots, D_{3}$ were completely determined by following the procedure outlined above. $D_{0}$ can be calculated in this way because the processdependent anomalous-dimension matrices in (76) and (77) are now known up to NNLO. With the same method, it was possible to calculate the scale dependence of $\delta$-function coefficient $C_{0}$. The function $R(z)$ is finite for $z \rightarrow 1$. The computation of the scale-independent part of $C_{0}$ requires the knowledge of the hard and soft functions at two-loop order. As long as these are missing, there is an ambiguity in $C_{0}$, associated with the fact that it is possible to normalize the scale-dependent logarithms in an arbitrary way, i.e.

$$
\ln \left(\frac{\mu_{0}^{2}}{\mu^{2}}\right)=\ln \left(\frac{\mu_{1}^{2}}{\mu^{2}}\right)+\ln \left(\frac{\mu_{0}^{2}}{\mu_{1}^{2}}\right) .
$$

Scale-independent terms proportional to the second logarithm on the right-hand side can be absorbed into the unknown $\mu$-independent part of the coefficient function $C_{0}$. Therefore, our numerical results for $C_{0}$ depend on the choice of the second mass scale, which appears in the scale-dependent logarithms; we indicate this second scale by $\mu_{0}$. In the next section we shall consider two different choices for $\mu_{0}: \mu_{0}=M$ (scheme A), and $\mu_{0}=m_{t}$ (scheme B). The situation is summarized in the following formula:

$$
C_{0}= \begin{cases}\sum_{i=0}^{4} c_{n}^{\mathrm{A}} \ln ^{n} \frac{M^{2}}{\mu^{2}} & (\text { scheme } \mathrm{A}) \\ \sum_{i=0}^{4} c_{n}^{\mathrm{B}} \ln ^{n} \frac{m_{t}^{2}}{\mu^{2}} & (\text { scheme B) }\end{cases}
$$

The coefficients $c_{n}^{\mathrm{A}}$ and $c_{n}^{\mathrm{B}}(n=1,2,3)$ are known, while $c_{0}^{\mathrm{A}}$ and $c_{0}^{\mathrm{B}}$ are unknown. In [56], the explicit expressions for the coefficients $D_{0}, \ldots, D_{3}$ and $C_{0}$ were collected in a Mathematica file, which can be downloaded from the arXiv version of that work.

\section{Phenomenological applications}

In this section we perform a numerical study of the invariant mass distribution, forwardbackward asymmetry, and total cross section at the Tevatron and LHC. The main purpose is to investigate the impact of the NNLL resummation and the approximate NNLO corrections on the central values and perturbative uncertainties in these observables. The higher-order corrections computed in this paper are limited to leading order in the threshold expansion, whereas the exact NLO results in fixed order also contain subleading terms in $(1-z)$. To make optimal use of our results, we match them onto the NLO fixed-order expressions in such a way that these subleading corrections are fully taken into account. For the resummed results, 
NLO+NNLL accuracy can be achieved by evaluating differential cross sections according to

$$
\begin{aligned}
d \sigma^{\mathrm{NLO}+\mathrm{NNLL}} & \left.\equiv d \sigma^{\mathrm{NNLL}}\right|_{\mu_{h}, \mu_{s}, \mu_{f}}+\left.d \sigma^{\mathrm{NLO}, \text { subleading }}\right|_{\mu_{f}} \\
& \left.\equiv d \sigma^{\mathrm{NNLL}}\right|_{\mu_{h}, \mu_{s}, \mu_{f}}+\left(\left.d \sigma^{\mathrm{NLO}}\right|_{\mu_{f}}-\left.d \sigma^{\mathrm{NLO}, \text { leading }}\right|_{\mu_{f}}\right),
\end{aligned}
$$

where $d \sigma^{\mathrm{NLO}}$ is the exact result in fixed order, and $\left.\left.d \sigma^{\mathrm{NLO} \text {, leading }}\right|_{\mu_{f}} \equiv d \sigma^{\mathrm{NNLL}}\right|_{\mu_{h}=\mu_{s}=\mu_{f}}$ captures

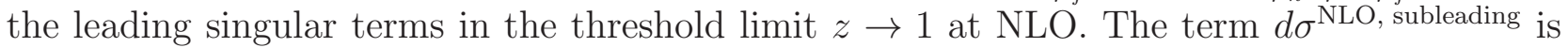
of subleading order in $(1-z)$ and ensures that the total result reduces to the exact fixed-order result when all the scales are set equal. It also makes the result invariant under variations of the factorization scale, up to terms at NNLO in the perturbative expansion, even at subleading order in $(1-z)$. To obtain approximate NNLO results in fixed order, we simply add the NNLO correction onto the exact NLO results, i.e.,

$$
d \sigma^{\mathrm{NNLO}, \text { approx }}=d \sigma^{\mathrm{NLO}}+d \sigma^{(2), \text { approx }},
$$

where $d \sigma^{(2)}$, approx is the NNLO correction to the differential cross section obtained using the coefficient function (99), in either scheme A or B described at the end of the previous section.

It is important to examine the relative size of the leading and subleading terms in (102). If the subleading terms were comparable in size to the leading ones, it would make little sense to resum the logarithms in $(1-z)$, which we have discussed so far, or to construct approximate fixed-order formulas at NNLO which capture only the effects of the singular terms. The naive expectation would be that the singular terms are dominant only when $\tau=M^{2} / s \rightarrow 1$, since then the integrand in (5) is needed only in the $z \rightarrow 1$ limit and the less singular terms are clearly subleading. However, the most interesting region for phenomenology ranges from $M \sim 2 m_{t}$ to around $1 \mathrm{TeV}$ at the Tevatron and up to several $\mathrm{TeV}$ at the LHC, which corresponds to $\tau<0.3$ (at most). For the leading-order singular terms to be dominant, it is necessary that the parton luminosity functions $f_{i j}(\tau / z, \mu)$ fall off sufficiently fast for $\tau / z \rightarrow 1$ that only the largest values of $z$ give significant contributions to the integrand, an effect referred to in [64-66] as dynamical threshold enhancement. The study of the invariant mass spectrum in [56] indicated that such an enhancement actually does take place in the range of invariant mass from near the peak of the distribution at $M \sim 380 \mathrm{GeV}$ up to much higher values. This is illustrated in Figure 2, which shows the invariant mass distributions at the Tevatron and LHC predicted using different approximations in fixed-order perturbation theory. The difference between the boundaries of the dark NLO bands and the dashed lines is due to the

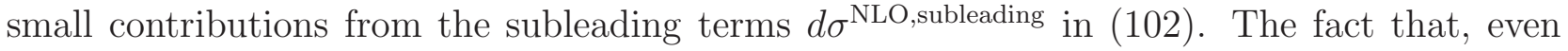
at these relatively low values of $M$, the leading terms provide a very good approximation to the full NLO result provides a strong motivation to study within our formalism higherorder corrections to integrated quantities such as the total cross section and forward-backward asymmetry, which receive their dominant contributions from low values of the invariant mass.

We will always do the matching onto fixed-order results as in (102) and (103), when the goal is to provide quantitative phenomenological predictions. Such a matching is straightforward for integrated quantities such as the total cross section and forward-backward asymmetry, since the NLO results in fixed order are available in analytic form. For the invariant mass 

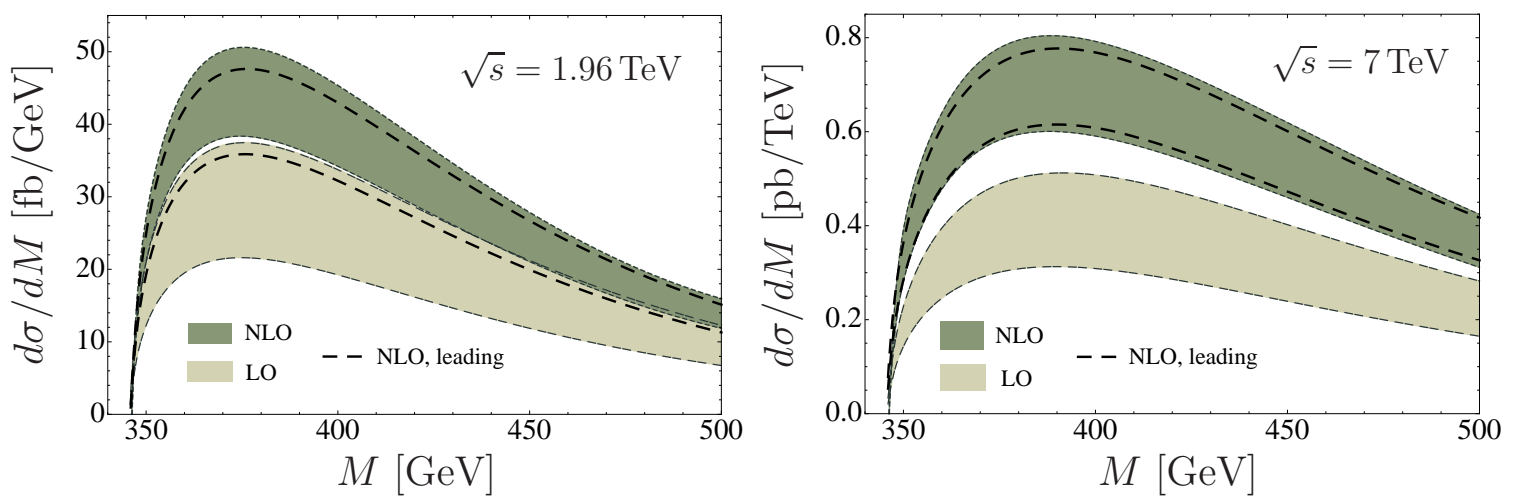

Figure 2: Fixed-order predictions for the invariant mass spectrum at LO (light bands) and NLO (dark bands) for the Tevatron (left) and LHC (right). We use MSTW2008NLO PDFs [87] with $\alpha_{s}\left(M_{Z}\right)=0.120$. The width of the bands reflects the uncertainty of the spectrum under variations of the matching and factorization scales. The dashed lines refer to the leading terms in the threshold expansion.

distribution, on the other hand, the fixed-order NLO results are available in the form of Monte Carlo programs such as MCFM [88]. This makes it difficult to get accurate values of the topquark pair invariant mass spectrum at high $M$, where the differential cross section is small, and makes it impractical to calculate the spectrum with the scale choice $\mu_{f}=M$ used in the next section, since doing so would require to run the program separately at each point in $\mu_{f}$. (Monte Carlo programs generate the invariant mass spectrum by first producing a set of events for a given $\mu_{f}$, and then grouping them into bins in $M$ ). When we study certain aspects of the invariant mass distribution in Section 6.1, we will take the NLO correction in the threshold approximation, so that (102) and (103) are evaluated with $d \sigma^{\mathrm{NLO}} \rightarrow d \sigma^{\mathrm{NLO} \text {, leading. }}$ This is still a good approximation to the full NLO result, and allows us to study the qualitative behavior of the invariant mass spectrum with $\mu_{f}=M$ over a large range of $M$, as well as PDF uncertainties, in a simple way. For this purpose, we also define an NNLO approximation which includes only the singular terms at threshold in the NLO correction:

$$
d \sigma^{\mathrm{NNLO}, \text { leading }}=d \sigma^{\mathrm{NLO}, \text { leading }}+d \sigma^{(2), \text { approx }} .
$$

\subsection{Invariant mass distribution: Systematic studies}

The invariant mass distribution is obtained by integrating the doubly differential rate over the range $-1<\cos \theta<1$. The resummed results (92) depend on the three scales $\mu_{s}, \mu_{h}$, and $\mu_{f}$, and to give a numerical result we must first specify how to choose them. In the similar cases of Drell-Yan [64] and Higgs production [65,66] at threshold, the soft and hard scales were chosen by examining the contributions of the one-loop soft and hard matching coefficients as functions of the scales $\mu_{s}$ and $\mu_{h}$, and then choosing default values of the scales in such a way as to minimize these corrections. We shall use this approach here, a small complication being the extra dependence on the kinematic variable $\cos \theta$ in (92). For the analysis of this section we use the default set of MSTW2008NNLO PDFs [87], take $\alpha_{s}\left(M_{Z}\right)=0.117$ with three-loop 

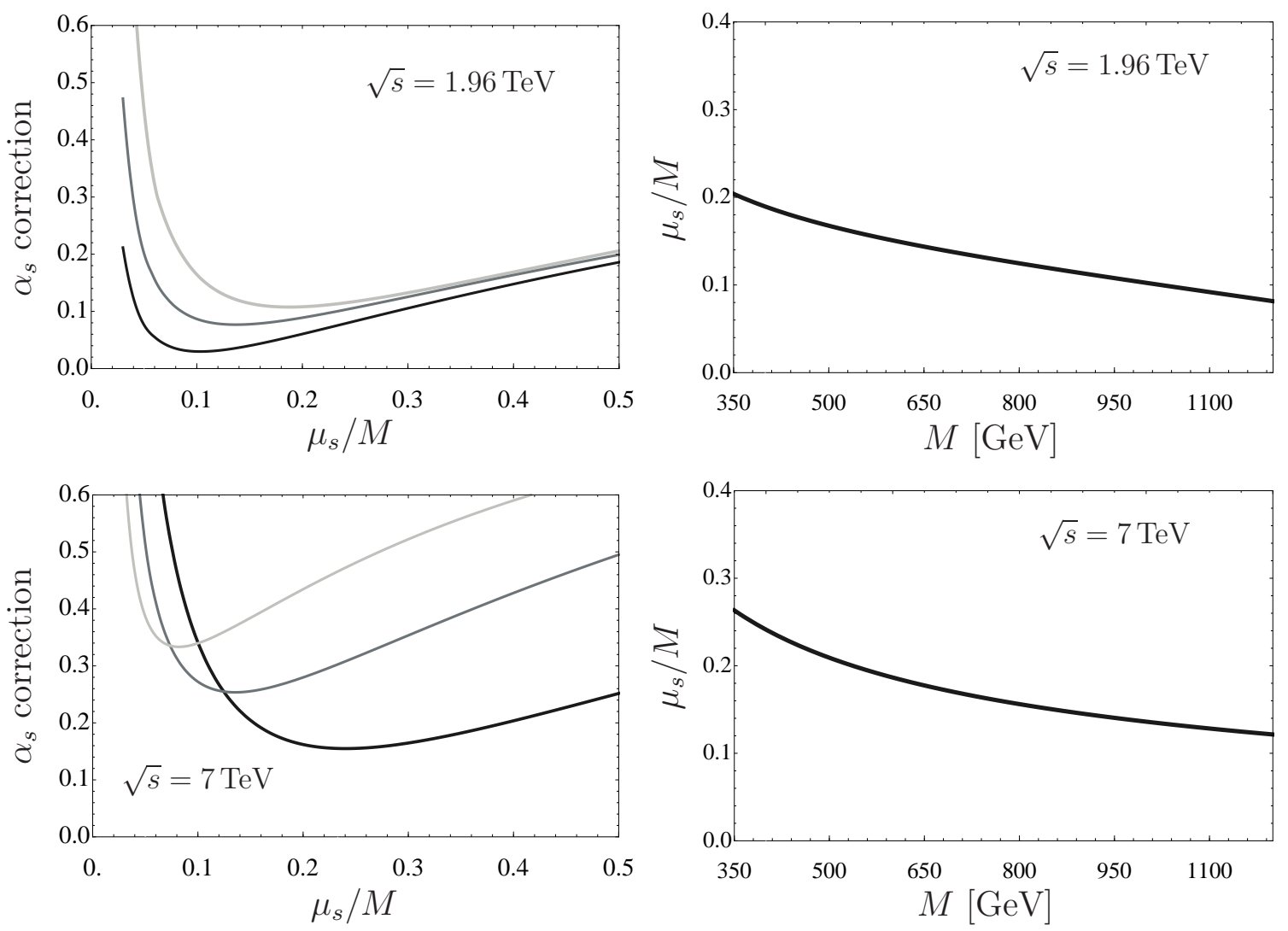

Figure 3: Top left: Ratio of the one-loop correction from the soft function over the leadingorder result for top pair production at the Tevatron, as a function of $\mu_{s} / M$, for $M=400 \mathrm{GeV}$ (dark), $M=700 \mathrm{GeV}$ (medium), and $M=1000 \mathrm{GeV}$ (light). Top right: The scale $\mu_{s} / M$ determined by the point where the one-loop correction from the soft function is minimal, as a function of the invariant mass $M$. Bottom: Analogous plots for the LHC, but with $M=400 \mathrm{GeV}$ (dark), $M=1000 \mathrm{GeV}$ (medium), and $M=2000 \mathrm{GeV}$ (light).

running in the $\overline{\mathrm{MS}}$ scheme with five active flavors, and employ the value $m_{t}=173.1 \mathrm{GeV}$ for the top-quark mass defined in the pole scheme. Using a fixed set of PDFs helps to elucidate more clearly the behavior of the perturbative expansion of the hard-scattering kernels in higher orders of perturbation theory. When presenting our phenomenological results in Sections 6.26.5, we will change the PDF sets according to the order of perturbation theory employed, and we will also study the theoretical uncertainties related to the parameterization of the PDFs. Finally, in Section 6.6, we will explore different schemes for the definition of the top-quark mass and study their impact on the phenomenological results.

\subsubsection{Determination of the matching and factorization scales}

We begin by examining the corrections from the NLO soft matching coefficient as a function of $\mu_{s}$. We isolate this contribution by picking out the piece of the NNLL approximation to (92) proportional to $\widetilde{\boldsymbol{s}}^{(1)}$, evaluating the differential cross section using only this piece, and dividing 

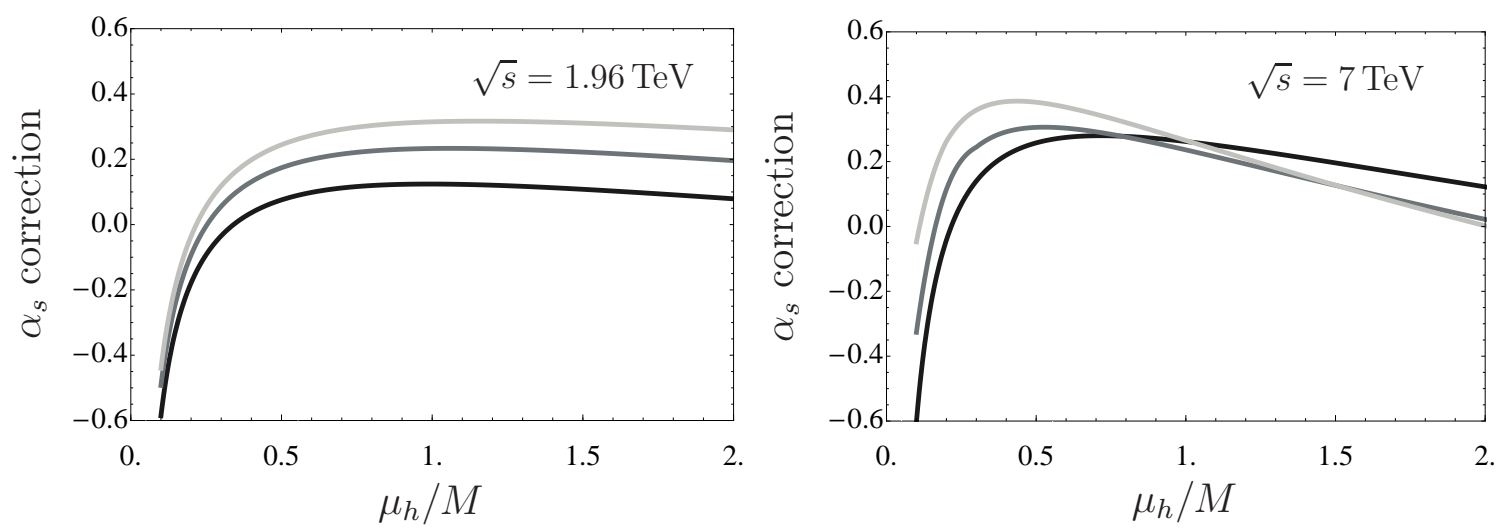

Figure 4: Left: Ratio of the one-loop correction the from hard function over the leadingorder result at the Tevatron, as a function of $\mu_{h} / M$, at $M=400 \mathrm{GeV}$ (dark), $M=700 \mathrm{GeV}$ (medium), and $M=1000 \mathrm{GeV}$ (light). Right: Analogous plot for the LHC, with $M=400 \mathrm{GeV}$ (dark), $M=1000 \mathrm{GeV}$ (medium), and $M=2000 \mathrm{GeV}$ (light).

the result by that at NLL, for the choice $\mu_{f}=\mu_{h}=M$. The results are shown in the left-hand plots of Figure 3 for the Tevatron and the LHC with $\sqrt{s}=7 \mathrm{TeV}$, for several different values of $M$. We note that the corrections are larger at the LHC than at the Tevatron, especially at high values of $M$. This behavior appears to be a property of the gluon channel, which gives the dominant contribution at the LHC. The correction is generally at its minimum between $M / 4$ and $M / 10$, and moves to lower values of $\mu_{s}$ at higher values of $M$. The exact position of the minimum as a function of $M$ is shown in the right-hand plots of Figure 3. To a good approximation, the numerical results for $\mu_{s}$ can be fitted by the function

$$
\mu_{s}^{\mathrm{def}}=\frac{M(1-\tau)}{\left(a+b \tau^{1 / 4}\right)^{c}}
$$

with $a=-33, b=150$, and $c=0.46$ for the Tevatron, and $a=-1.3, b=23$, and $c=0.98$ for the LHC at $\sqrt{s}=7 \mathrm{TeV}$. In Section 6.3 we will also study the total cross section at the LHC with $\sqrt{s}=10,14 \mathrm{TeV}$, and in those cases we use $a=0.95, b=6.7$, and $c=1.6$.

The most appropriate choice of the hard scale $\mu_{h}$ is not immediately apparent, since the invariant mass spectrum depends on the two hard scales $m_{t}$ and $M$. As a guide to an appropriate choice we look at the size of the correction from the hard matching function for different choices of $\mu_{h}$. We show in Figure 4 the correction obtained by isolating the contribution of $\boldsymbol{H}^{(1)}$ to the differential cross section at NNLL, and dividing it by the NLL result, for the choice $\mu_{f}=M$ and $\mu_{s}$ determined according to (105). We see that at lower values of $\mu_{h}$ closer to $m_{t}$ the correction typically gets smaller and can even become negative. In this lower range of $\mu_{h}$, however, the correction depends very strongly on the scale. The results are more stable in the range $M / 2<\mu_{h}<2 M$, where the correction is generally below $30 \%$ at the Tevatron and between $20-40 \%$ at the LHC. In what follows we shall choose $\mu_{h}=M$ by default, in order to avoid the instability at lower $\mu_{h}$. In the case of Higgs production, a negative hard scale squared $\mu_{h}^{2} \sim-m_{H}^{2}-i \epsilon$ was chosen to minimize the logarithms arising from time-like kinematics $[65,66]$. In the $t \bar{t}$ case, however, there are both time-like and space-like momentum 

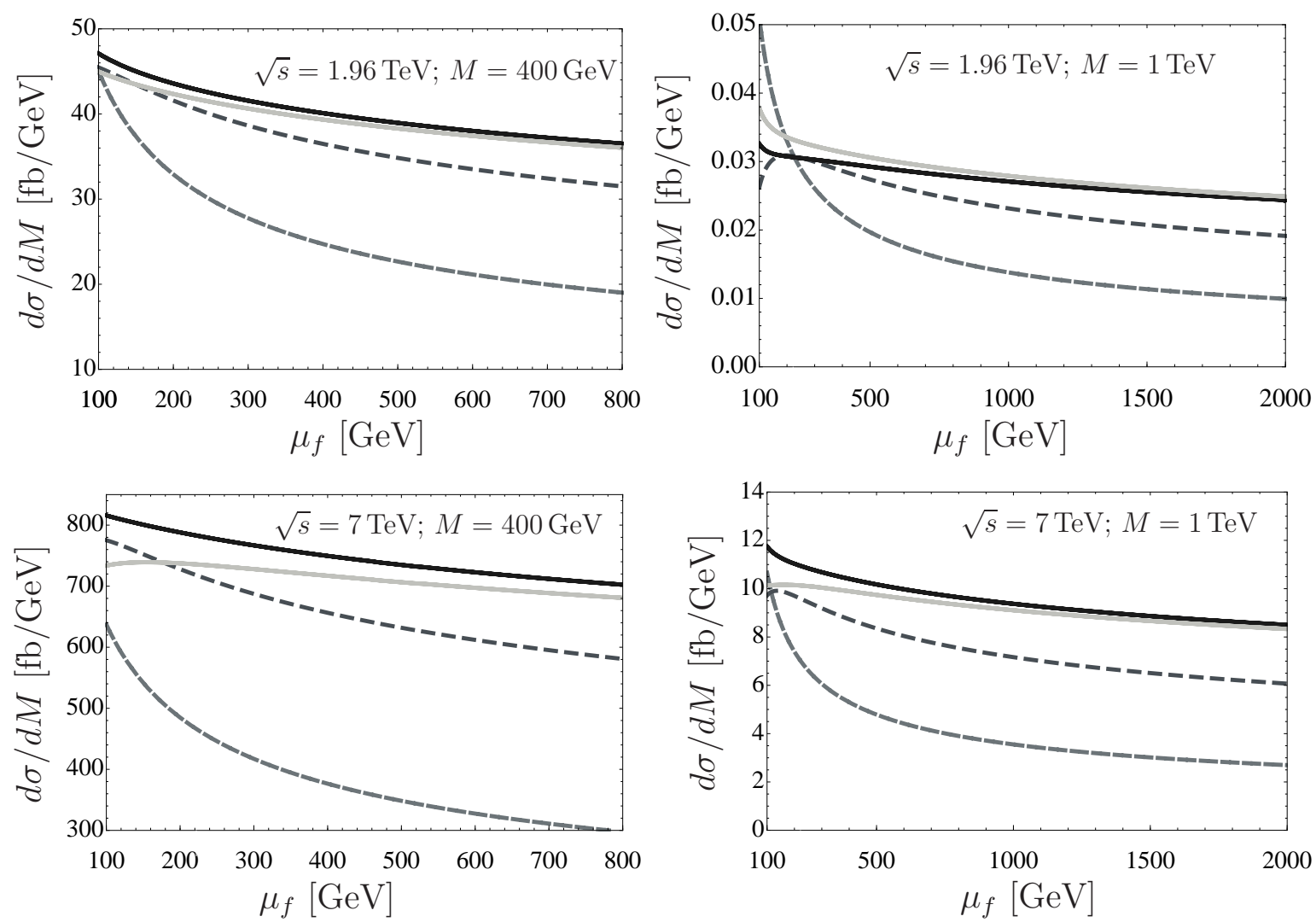

Figure 5: Dependence of $d \sigma / d M$ at the Tevatron (left) and LHC (right) on the scale $\mu_{f}$ in fixed-order perturbation theory. The dashed-dotted lines show $d \sigma^{\mathrm{LO}}$, the dashed lines $d \sigma^{\mathrm{NLO}}$, leading, and the dark (light) solid lines the approximate threshold expansion (104) at NNLO in scheme A (scheme B).

invariants, and it is not straightforward to tell which point in the complex plane should be chosen to minimize the logarithms. We have thus investigated the choice $\mu_{h}^{2}=M^{2} e^{i \phi_{h}}$ with $\phi_{h}$ varied between $-\pi$ and $\pi$. The results show that at the LHC, the correction is smallest for $\phi_{h} \sim-\pi$, and is about $10 \%$ compared to $20 \%$ at $\phi_{h} \sim 0$. At the Tevatron, however, the minimal correction is obtained for values close to $\phi_{h} \sim 0$. In view of this, and since the corrections are in any case not very large, we will not go into this complication in our numerical analyses below.

Finally, we must choose a default value for the factorization scale $\mu_{f}$ in both the resummed and fixed-order results. To do so, we study the behavior of the cross sections as a function of this scale. For the fixed-order results, the invariant mass spectrum as a function of $\mu_{f}$ at $M=400 \mathrm{GeV}$ and $M=1 \mathrm{TeV}$ is shown in Figure 5. For the moment we do not match the results onto fixed-order perturbation theory at NLO, using instead the threshold expansion $d \sigma^{\mathrm{NLO}}$, leading and $d \sigma^{\mathrm{NNLO}}$, leading. As a result, our predictions are not strictly independent of the scale $\mu_{f}$, but a slight scale dependence enters via subleading terms in $(1-z)$. At $M=400 \mathrm{GeV}$ the approximate NNLO formulas differ from each other less at $\mu_{f} \sim 400 \mathrm{GeV}$ than at $\mu_{f} \sim m_{t}$. The same is true at $M=1 \mathrm{TeV}$, but in this case the results become very unstable at $\mu_{f} \sim m_{t}$. 

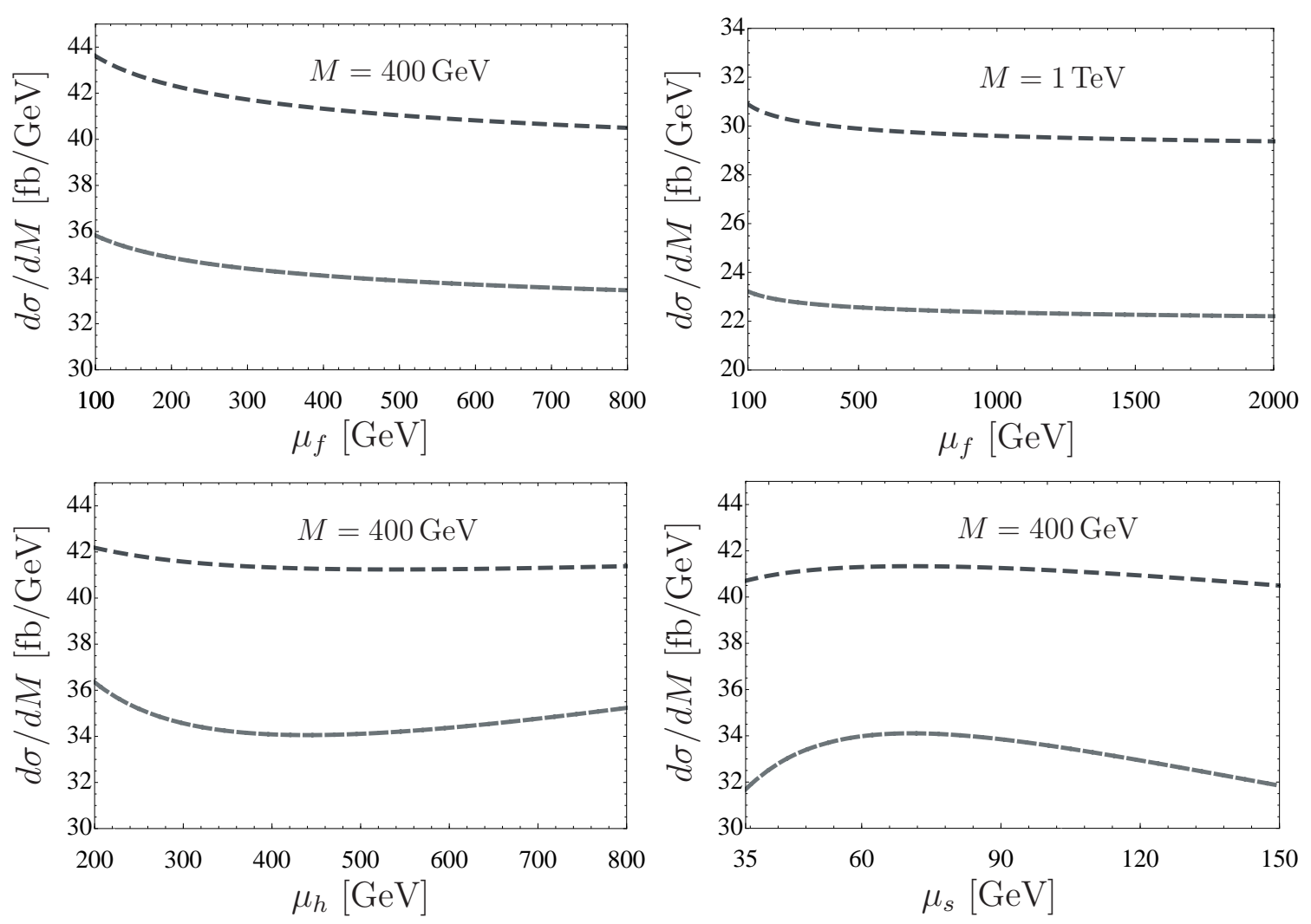

Figure 6: Dependence of $d \sigma / d M$ at the Tevatron on the scales $\mu_{h}, \mu_{s}$, and $\mu_{f}$ in resummed perturbation theory. The default choices are $\mu_{h}=\mu_{f}=M$, and $\mu_{s}$ according to (105). The dashed-dotted lines refer to NLL, the dashed to NNLL.

It therefore seems more appropriate to make the choice $\mu_{f} \sim M$ when studying the invariant mass spectrum. The resummed results at $M=400 \mathrm{GeV}$ and $M=1 \mathrm{TeV}$ as a function of $\mu_{f}$, with $\mu_{h}=M$ and $\mu_{s}$ as in (105), are shown in the upper two plots of Figure 6, for the case of the Tevatron (plots for the LHC would look very similar). Again, the results at $\mu_{f} \sim M$ are more stable than at $\mu_{f} \sim m_{t}$, although compared to the fixed-order results the difference is less pronounced. We will thus make the choice $\mu_{f}=M$ by default in the resummed result.

Having chosen default values for the scales, we now discuss in more detail the behavior of the fixed-order and resummed predictions for the invariant mass distribution. We have already seen how the results depend on the scale $\mu_{f}$. In the lower two plots of Figure 6 , we show the dependence of the resummed results at $M=400 \mathrm{GeV}$ on the scales $\mu_{h}$ and $\mu_{s}$. The results as a function of these two scales are significantly more stable at NNLL than at NLL.

\subsubsection{Convergence of the perturbation series}

An interesting difference between the fixed-order and resummed results is that the perturbative uncertainties and the size of the higher-order corrections in the resummed results depend much less on the value of $M$. This is seen in Figure 7, where we show the $K$ factors and uncertainties in the invariant mass spectrum as a function of $M$, comparing fixed-order results with the 

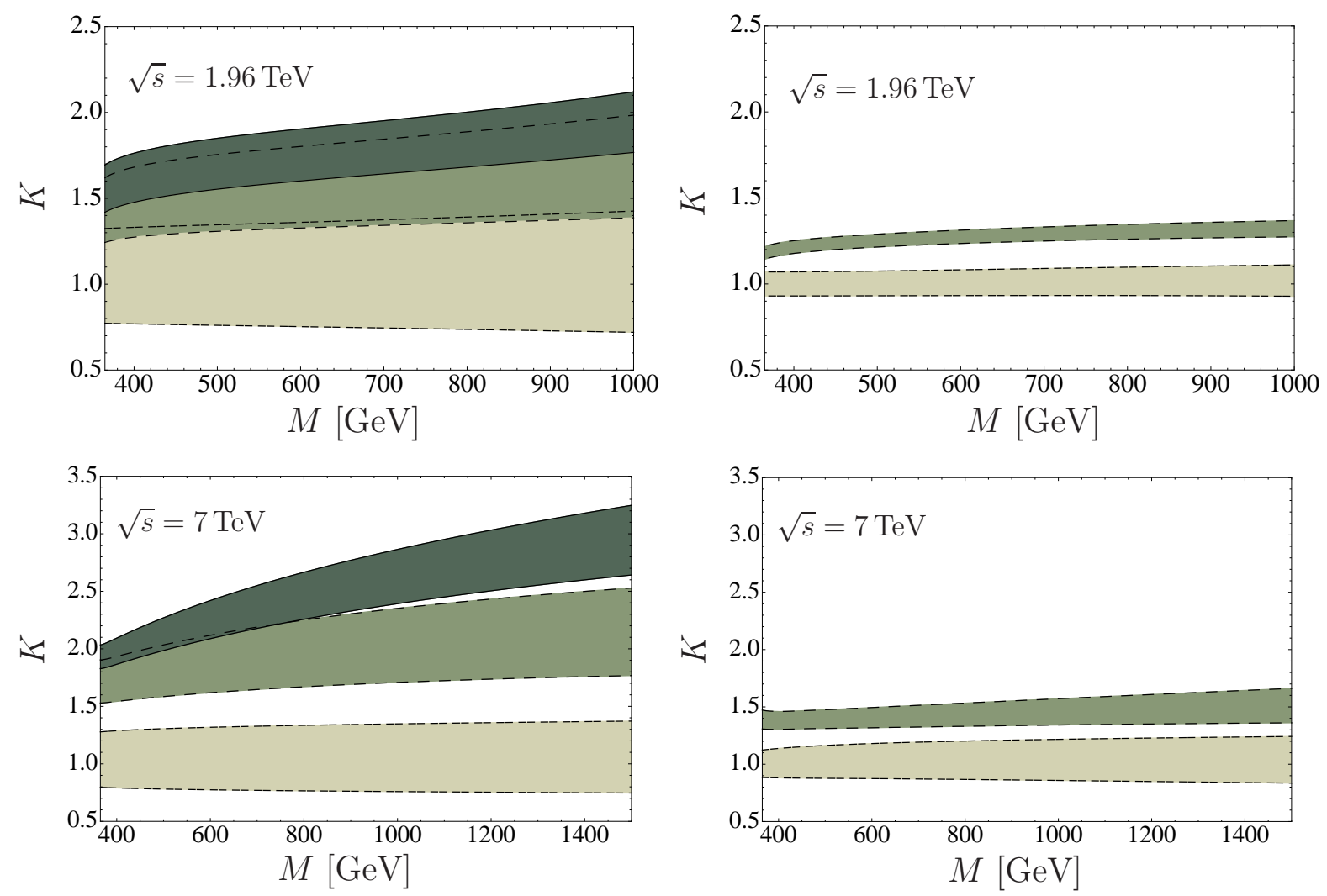

Figure 7: $K$ factors $(d \sigma / d M) /\left(d \sigma^{\mathrm{LO} \text {, def }} / d M\right)$ in fixed-order perturbation theory (left) and $(d \sigma / d M) /\left(d \sigma^{\mathrm{NLL}, \text { def }} / d M\right)$ in resummed perturbation theory (right), for the Tevatron (top) and LHC (bottom). The light bands in the fixed-order (resummed) results show LO (NLL) results, the medium bands show NLO leading (NNLL) results, and the dark bands in the fixed-order results refer to the approximate threshold expansion (104) at NNLO in scheme A. The width of the bands reflects the uncertainties associated with variations of the scales, as described in the text.

resummed ones. The bands in fixed order reflect the uncertainty associated with varying the factorization scale around its default value $\mu_{f}=M$ by a factor of two. Here and in the following figures, for the approximate NNLO formulas we show results only in scheme A; those in scheme B look very similar. To make the bands in resummed perturbation theory, we first obtain uncertainties associated with the scales $\mu_{f}, \mu_{h}$, and $\mu_{s}$ by varying them individually up and down by a factor of two from their default values at each point in $M$, with the other two scales held fixed. We then obtain a total error by adding the three uncertainties obtained this way in quadrature. At the Tevatron, the $K$ factors in resummed perturbation theory have smaller uncertainties and depend only weakly on $M$, compared to fixed order. The same is true at the LHC, although at small $M$ the approximate NNLO results and the resummed ones have comparable uncertainties.

To illustrate more precisely the quantitative differences between the various perturbative approximations to the invariant mass spectrum, we show in Table 1 the exact numerical values of the spectrum at the points $M=400 \mathrm{GeV}$ and $M=1000 \mathrm{GeV}$. We have assigned uncertain- 


\begin{tabular}{|l|c|c|}
\hline Tevatron & $M=400 \mathrm{GeV}[\mathrm{fb} / \mathrm{GeV}]$ & $M=1 \mathrm{TeV}[\mathrm{fb} / \mathrm{GeV}]$ \\
\hline NLL & $34.1_{-2.4-1.2}^{+2.4+1.7}$ & $\left(22.4_{-1.6-1.3}^{+2.5+1.7}\right) \cdot 10^{-3}$ \\
NLO, leading & $36.5_{-5.0-1.4}^{+5.1+1.9}$ & $\left(23.2_{-4.0}^{+4.2+1.9}\right) \cdot 10^{-3}$ \\
NNLL & $41.3_{-1.2-1.4}^{+1.3+1.9}$ & $\left(29.6_{-1.1-1.6}^{+1.0+2.2}\right) \cdot 10^{-3}$ \\
NNLO, leading (scheme A) & $40.1_{-3.6-1.4}^{+3.5+1.9}$ & $\left(27.1_{-2.7-1.6}^{+2.2+2.1}\right) \cdot 10^{-3}$ \\
NNLO, leading (scheme B) & $39.3_{-3.3-1.4}^{+3.0+1.9}$ & $\left(27.9_{-3.0}^{+2.7+2.2}\right) \cdot 10^{-3}$ \\
\hline \hline LHC $(\sqrt{s}=7 \mathrm{TeV})$ & $M=400 \mathrm{GeV}[\mathrm{fb} / \mathrm{GeV}]$ & $M=1 \mathrm{TeV}[\mathrm{fb} / \mathrm{GeV}]$ \\
\hline NLL & $558_{-68}^{+78+20}$ & $7.43_{-1.07}^{+1.61+0.69}$ \\
NLO, leading & $656_{-76-27}^{+72+26}$ & $7.17_{-1.10-0.69}^{+1.19+0.69}$ \\
NNLL & $775_{-47-31}^{+39+30}$ & $10.83_{-0.87-1.03}^{+0.84+1.01}$ \\
NNLO, leading (scheme A) & $750_{-47-30}^{+38+29}$ & $9.38_{-0.87-0.90}^{+0.82+0.90}$ \\
NNLO, leading (scheme B) & $717_{-36-29}^{+20+28}$ & $9.11_{-0.78-0.86}^{+0.63+0.86}$ \\
\hline
\end{tabular}

Table 1: Values for $d \sigma / d M$ for $M=400 \mathrm{GeV}$ and $M=1 \mathrm{TeV}$ at the Tevatron and the LHC at $\sqrt{s}=7 \mathrm{TeV}$. The first error refers to perturbative scale uncertainties, the second to PDF uncertainties, see text for a detailed explanation.

\begin{tabular}{|l|c|c|}
\hline Order & PDF set & $\alpha_{s}\left(M_{Z}\right)$ \\
\hline LO & MSTW2008LO & 0.139 \\
NLO, NLL & MSTW2008NLO & 0.120 \\
NNLO approx, NLO+NNLL & MSTW2008NNLO & 0.117 \\
\hline
\end{tabular}

Table 2: Order of the PDFs [87] and the corresponding values of the strong coupling used for the different perturbative approximations.

ties associated with variations of the various scales by factors of two up and down from their default values. To obtain a total scale uncertainty for the resummed results, we have added the uncertainties associated with variations of $\mu_{h}, \mu_{s}$, and $\mu_{f}$ in quadrature. We have also included uncertainties associated with the PDFs, by using the set of MSTW2008NNLO PDFs from [87] at 90\% confidence level (CL). The perturbative scale uncertainties are smaller or comparable than those from the PDFs only once the NNLL or approximate NNLO corrections are taken into account. For the practical reasons explained earlier, we have not matched the higher-order results with the fixed-order NLO results. However, the threshold approximation works rather well. For reference, at the Tevatron the exact NLO results are $\left(38.6_{-5.2}^{+5.1}\right) \mathrm{fb} / \mathrm{GeV}$ for $M=400 \mathrm{GeV}$ and $\left(24.8_{-4.8}^{+4.5}\right) \cdot 10^{-3} \mathrm{fb} / \mathrm{GeV}$ for $M=1000 \mathrm{GeV}$, while at the LHC they are $\left(654_{-89}^{+98}\right) \mathrm{fb} / \mathrm{GeV}$ for $M=400 \mathrm{GeV}$ and $\left(6.84_{-1.11}^{+1.40}\right) \mathrm{fb} / \mathrm{GeV}$ for $M=1000 \mathrm{GeV}$. The deviations from the leading NLO terms shown in the second line in both parts of the table are smaller than $7 \%$ for the Tevatron and $5 \%$ for the LHC. 

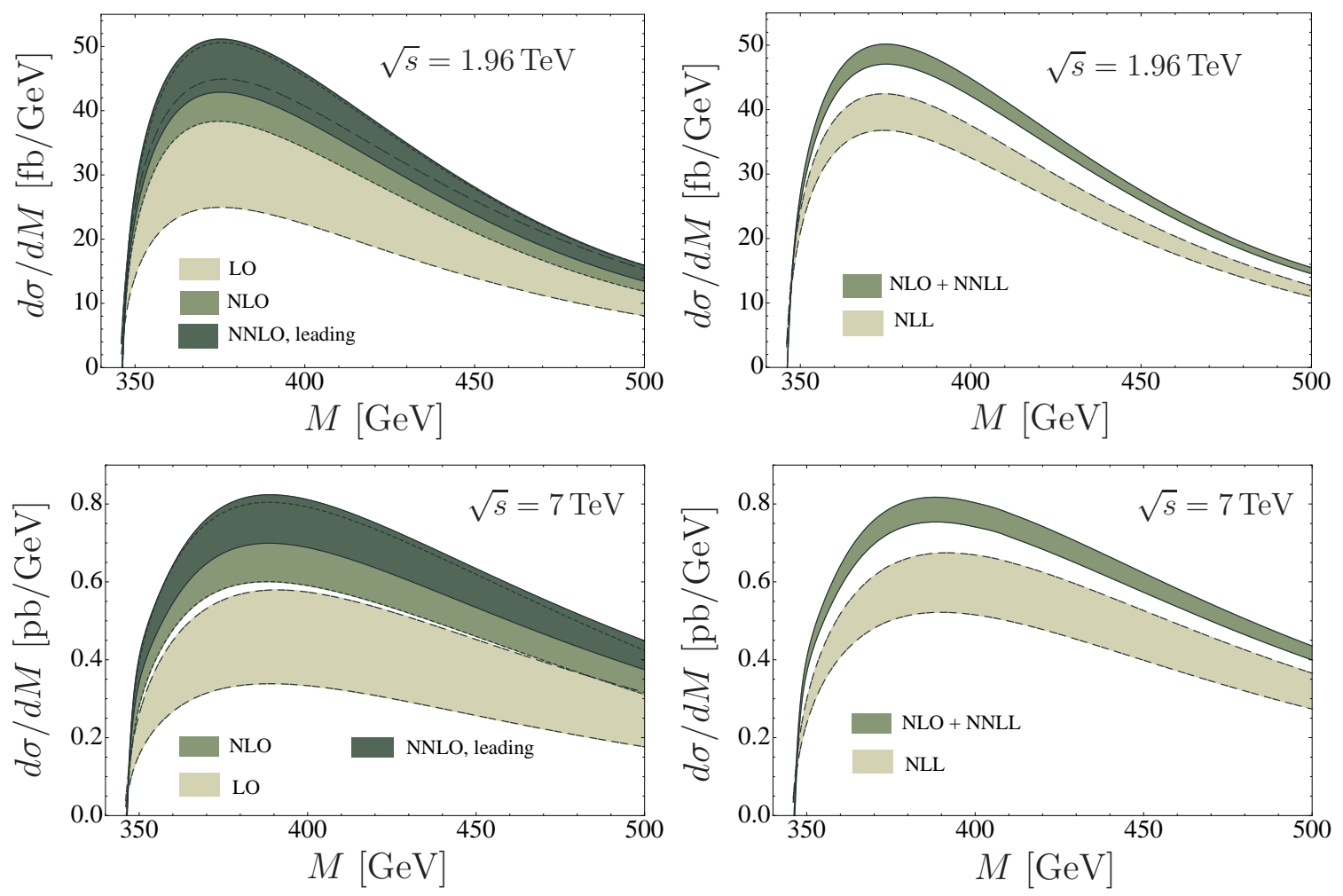

Figure 8: Left: Fixed-order predictions for the invariant mass spectrum at LO (light), NLO (darker), and approximate NNLO (dark bands) for the Tevatron (top) and LHC (bottom). Right: Corresponding predictions at NLL (light) and NLO+NNLL (darker bands) in resummed perturbation theory. The width of the bands reflects the uncertainty of the spectrum under variations of the matching and factorization scales, as explained in the text.

\subsection{Invariant mass distribution: Phenomenological results}

After these systematic studies, we now present our final results for the $t \bar{t}$ invariant mass distributions at the Tevatron and LHC. Here and below, we will use different sets of PDFs, as appropriate for the order of the perturbative approximation employed. Strictly speaking, theoretical predictions obtained in resummed perturbation theory would require PDF sets extracted from data using resummed predictions for the relevant cross sections; however, such PDF sets do not exist at present. Since our resummed expressions include the bulk of the perturbative corrections appearing one order higher in $\alpha_{s}$, we use NLO parton densities for the NLO and NLL approximations, and NNLO parton densities for the approximate NNLO and matched NLO+NNLL approximations, as summarized in Table 2. The associated running couplings $\alpha_{s}(\mu)$ are taken in the $\overline{\mathrm{MS}}$ scheme with five active flavors, using one-loop running at LO, two-loop running at NLO, and three-loop running at NNLO.

We begin by studying in more detail the invariant mass spectrum at relatively low values of $M$, where it is the largest, in fixed-order and resummed perturbation theory. Contrary to the previous section, we now match the results in resummed perturbation theory with the exact fixed-order results at NLO using the MCFM program, according to (102). In this way 

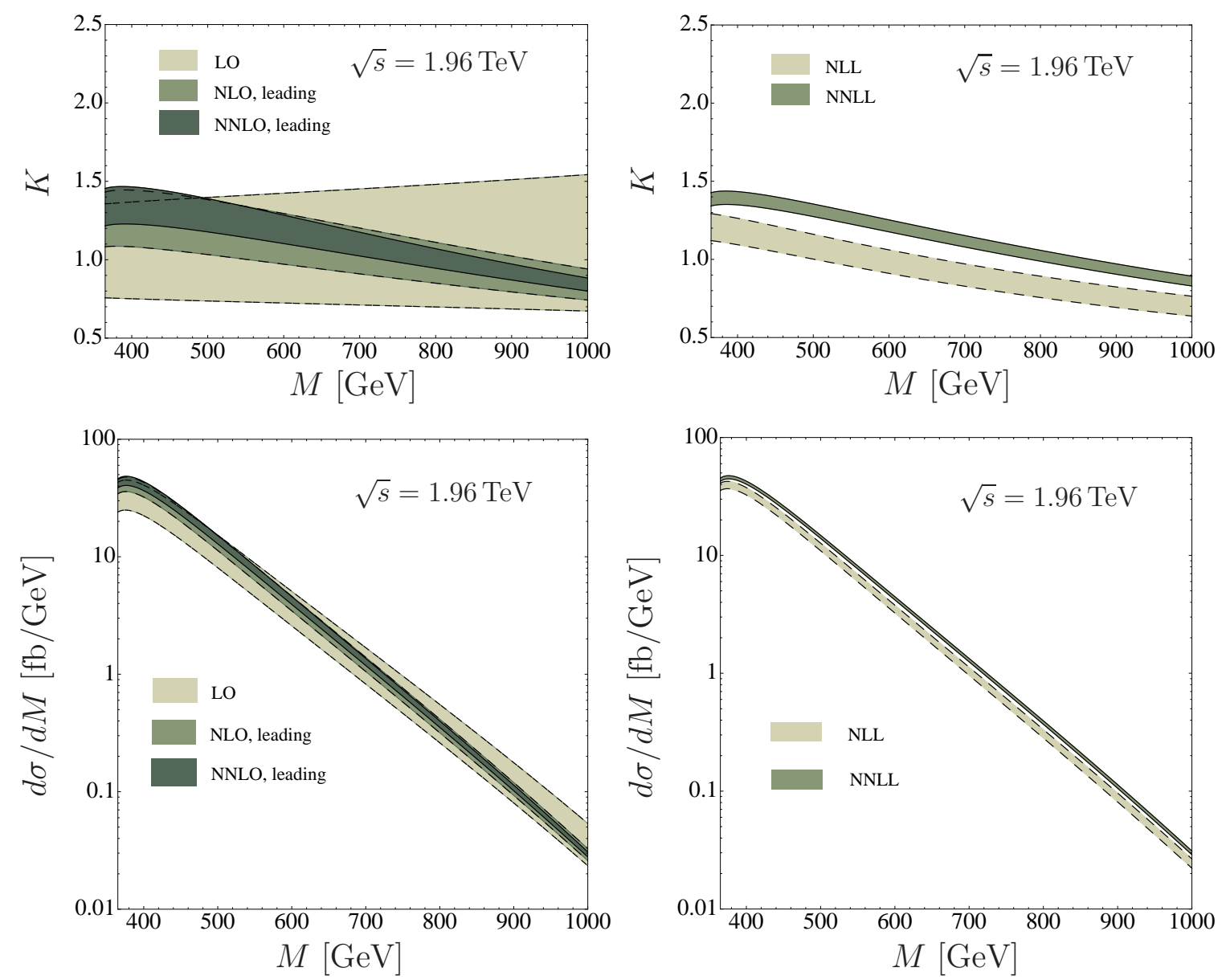

Figure 9: Left: Fixed-order predictions for the $K$ factor and invariant mass spectrum at LO (light), NLO (darker), and approximate NNLO (dark bands) for the Tevatron. Right: Corresponding predictions at NLL (light) and NLO+NNLL (darker bands) in resummed perturbation theory. The width of the bands reflects the uncertainty of the spectrum under variations of the matching and factorization scales, as explained in the text.

we obtain state-of-the-art predictions, which include everything known about the perturbative series for the spectrum. Our results are shown in Figure 8. The bands reflect uncertainties in scale variations according to the same procedure explained in the previous paragraph, but in this case with $\mu_{f}=400 \mathrm{GeV}$ by default. For the range of $M$ in the plot, this choice is very close to our preferred scheme $\mu_{f}=M$, but allows for a simple matching with the fixed-order results from MCFM. One sees that the perturbative uncertainty estimated by scale variations is by far the smallest at NLO+NNLL order.

We now consider the region of higher invariant masses, for which the dominance of the threshold terms is even more pronounced, as indicated by the convergence of the dark bands and dashed lines in Figure 2 toward higher $M$ values. Figure 9 shows our results for the Tevatron, both in fixed-order and resummed perturbation theory. Figure 10 shows the corresponding results for the LHC. It is impractical to match onto fixed-order results obtained 

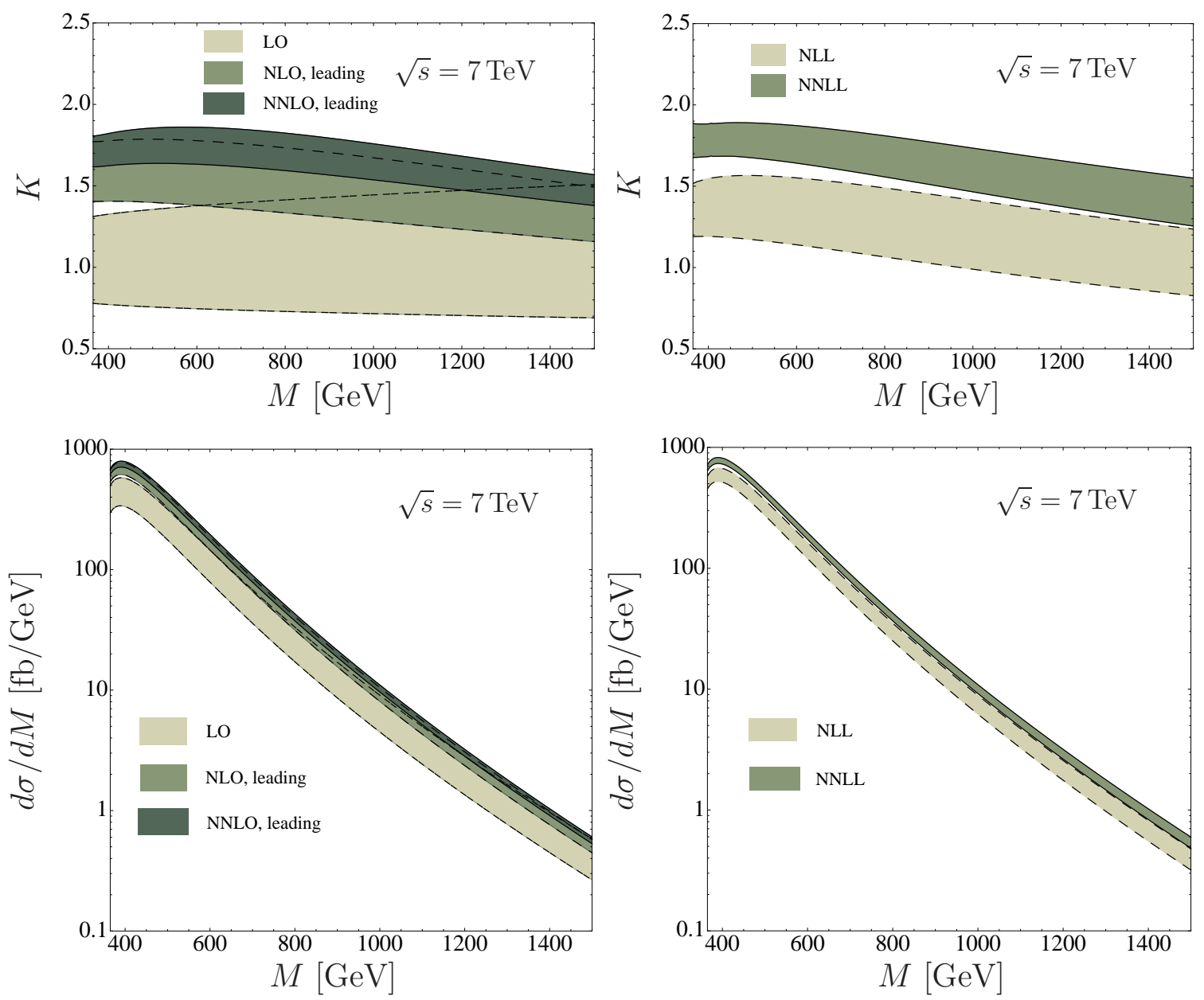

Figure 10: Left: Fixed-order predictions for the $K$ factor and invariant mass spectrum at LO (light), NLO (darker), and approximate NNLO (dark bands) for the LHC. Right: Corresponding predictions at NLL (light) and NLO+NNLL (darker bands) in resummed perturbation theory. The width of the bands reflects the uncertainty of the spectrum under variations of the matching and factorization scales, as explained in the text.

using the MCFM program in this case; however, the differences compared with the shown curves are so small that they would hardly be visible on the scales of the plots. The upper two plots show $K$ factors, which are defined as the ratio of the cross section to the default lowest-order prediction $d \sigma^{\mathrm{LO} \text {,def }} / d M$. Contrary to Figure 7 , we now use the same normalization in both fixed-order and resummed perturbation theory, so that the two spectra can more readily be compared to each other. The lower plots show the corresponding spectra directly. We observe similar behavior as in the low-mass region. The bands obtained in fixed-order perturbation theory become narrower in higher orders and overlap. The bands obtained in resummed perturbation theory are narrower than the corresponding ones at fixed order. The leading-order resummed prediction is already close to the final result.

The information contained in Figures 8-10 can be represented differently in terms of the 

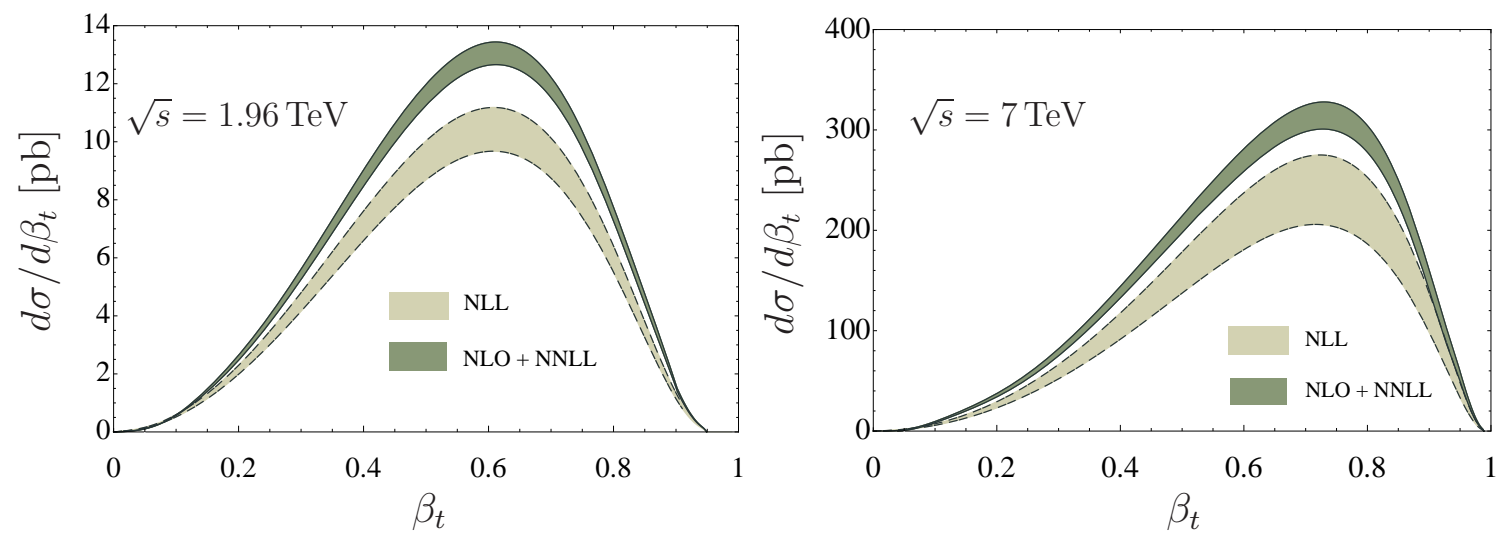

Figure 11: Distributions $d \sigma / d \beta_{t}$ at the Tevatron (left) and LHC (right).
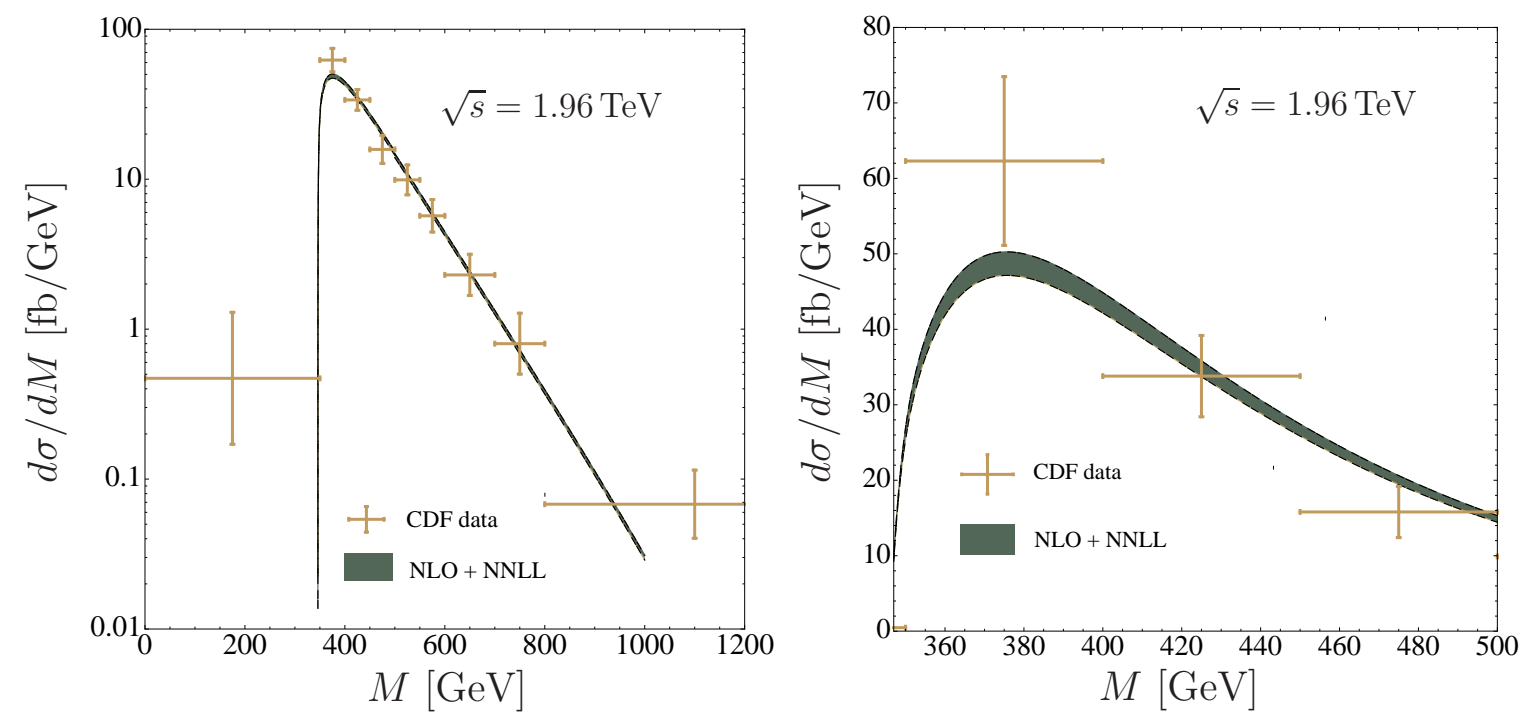

Figure 12: Comparison of the RG-improved predictions for the invariant mass spectrum with CDF data [9]. The value $m_{t}=173.1 \mathrm{GeV}$ has been used. No fit to the data has been performed.

very useful distribution $d \sigma / d \beta_{t}$, with $\beta_{t}$ defined as in (4). A simple change of variables yields

$$
\frac{d \sigma}{d \beta_{t}}=\frac{2 m_{t} \beta_{t}}{\left(1-\beta_{t}^{2}\right)^{\frac{3}{2}}} \frac{d \sigma}{d M}
$$

The resulting spectra for the Tevatron and LHC, obtained using RG-improved perturbation theory, are shown in Figure 11. As before, the distributions are normalized such that the area under the curves corresponds to the total cross section. Recall that the physical meaning of the variable $\beta_{t}$ is that of the 3 -velocity of the top quarks in the $t \bar{t}$ rest frame. The distributions show that the dominant contributions to the cross section arise from the region of relativistic top quarks, with velocities of order $0.4-0.8$ at the Tevatron and $0.5-0.9$ at the LHC. We will come back to the significance of this observation in the next section.

In Figure 12, we compare our RG-improved prediction for the invariant mass spectrum 
to a measurement of the CDF collaboration obtained using the "lepton + jets" decay mode of the top quark [9]. We observe an overall good agreement between our prediction and the measurement, especially for higher values of $M$. Apparently, there is no evidence of nonstandard resonances in the spectrum. The only small deviation from our prediction concerns the peak region of the distribution, shown in more details in the right plot. This deviation has also been observed in [9], where a Monte Carlo study of the SM expectation has been performed.

\subsection{Total cross section: Phenomenological results}

The total cross section is obtained in our approach by integrating numerically the doubly differential cross section in the ranges $-1<\cos \theta<1$ and $2 m_{t}<M<\sqrt{s}$. In this case it is a simple matter to match onto NLO in fixed-order perturbation theory, using the analytic results of [16]. To do this, however, we can no longer correlate the factorization scale $\mu_{f}$ with $M$, as we did when studying the invariant mass spectrum. Instead, we should resort to representative average values of $M$, which characterize the spectrum in the region yielding sizable contributions to the total cross section. One possibility is to take the location of the peak in the $d \sigma / d M$ distributions, which is $M_{\text {peak }} \approx 375 \mathrm{GeV}$ for the Tevatron and $M_{\text {peak }} \approx$ $388 \mathrm{GeV}$ for the LHC (see Figure 8). Another possibility is to take the average value $\langle M\rangle$ of the distributions, for which we find $\langle M\rangle \approx 445 \mathrm{GeV}$ for the Tevatron and $\langle M\rangle \approx 496 \mathrm{GeV}$ for the LHC. As previously, we take the fixed value $\mu_{f}=400 \mathrm{GeV}$ as our default choice. On the other hand, we are still free to choose the hard and soft scales as we have done so far and match with the fixed-order result as shown in (102). We display in Table 3 the central values and scale uncertainties for the total cross section obtained using this procedure. The results in resummed perturbation theory use $\mu_{h}=M$ and $\mu_{s}$ chosen according to (105) by default, and the uncertainties are obtained by varying these scales and the factorization scale $\mu_{f}$ up and down by a factor of two and adding the different uncertainties in quadrature. The perturbative uncertainties in the fixed-order results are obtained by varying the factorization scale up and down by a factor of two from its default value. In addition to the perturbative uncertainties, we also list the PDF uncertainties obtained by evaluating the cross section with the appropriate set of MSTW2008 PDFs at 90\% CL. As shown in Table 2, the LO cross sections are evaluated using LO PDF sets, the NLL and NLO cross sections using NLO PDF sets, and the NNLL and approximate NNLO cross sections using NNLO PDF sets. In the following tables, these different classes of predictions are separated by horizontal lines.

A few comments are in order concerning the results shown in the table. At NLO the cross sections $\sigma_{\mathrm{NLO}}$, leading evaluated using only the leading singular terms from the threshold expansion reproduce between 95\% (for the Tevatron) to almost 100\% (for the LHC) of the exact fixed-order result at the default values of the factorization scale. The subleading terms in $(1-z)$, obtained by integrating $d \sigma_{\mathrm{NLO}}$, subleading, contribute the remaining few percent. In other words, the singular terms capture about $85 \%$ of the NLO correction at the Tevatron and practically $100 \%$ of it at the LHC. We cannot say whether the threshold expansion works so well also at higher orders in perturbation theory, although this does not seem unreasonable. Our best prediction is obtained by matching the fixed-order result with the resummed result at NLO+NNLL accuracy and is highlight in gray. The effect of resummation is roughly a 


\begin{tabular}{|l|c|c|c|c|}
\hline & Tevatron & LHC $(7 \mathrm{TeV})$ & $\mathrm{LHC}(10 \mathrm{TeV})$ & $\mathrm{LHC}(14 \mathrm{TeV})$ \\
\hline$\sigma_{\mathrm{LO}}$ & $4.49_{-1.15-0.19}^{+1.71+0.24}$ & $84_{-20-5}^{+29+4}$ & $217_{-49-11}^{+70+10}$ & $495_{-107-24}^{+148+19}$ \\
\hline$\sigma_{\mathrm{NLL}}$ & $5.07_{-0.36-0.18}^{+0.37+0.28}$ & $112_{-14-5}^{+18+5}$ & $276_{-37-11}^{+47+10}$ & $598_{-94-19}^{+108+19}$ \\
$\sigma_{\mathrm{NLO}, \text { leading }}$ & $5.49_{-0.78-0.20}^{+0.78+0.31}$ & $134_{-17-7}^{+16+7}$ & $341_{-38-14}^{+34+14}$ & $761_{-75-26}^{+64+25}$ \\
$\sigma_{\mathrm{NLO}}$ & $5.79_{-0.80-0.22}^{+0.79+0.33}$ & $133_{-19-7}^{+21+7}$ & $341_{-46-15}^{+50+14}$ & $761_{-101}^{+105+26}$ \\
\hline$\sigma_{\mathrm{NLO}+\text { NNLL }}$ & $6.30_{-0.19-0.23}^{+0.19+0.31}$ & $149_{-7-8}^{+7+8}$ & $373_{-15-16}^{+17+16}$ & $821_{-42-31}^{+40+24}$ \\
$\sigma_{\mathrm{NNLO}, \text { approx }}$ (scheme A) & $6.14_{-0.53-0.23}^{+0.49+0.31}$ & $146_{-12-8}^{+13+8}$ & $369_{-30-16}^{+34+16}$ & $821_{-65-29}^{+11+27}$ \\
$\sigma_{\mathrm{NNLO}, \text { approx }}$ (scheme B) & $6.05_{-0.50-0.23}^{+0.43+0.31}$ & $139_{-9-7}^{+9+7}$ & $349_{-23-15}^{+23+15}$ & $773_{-50-27}^{+47+25}$ \\
\hline
\end{tabular}

Table 3: Results for the total cross section in pb, using the default choice $\mu_{f}=400 \mathrm{GeV}$. The first set of errors refers to perturbative uncertainties associated with scale variations, the second to PDF uncertainties. The most advanced prediction is the NLO+NNLL expansion highlighted in gray.

\begin{tabular}{|l|c|c|c|c|}
\hline & Tevatron & $\mathrm{LHC}(7 \mathrm{TeV})$ & $\mathrm{LHC}(10 \mathrm{TeV})$ & $\mathrm{LHC}(14 \mathrm{TeV})$ \\
\hline$\sigma_{\mathrm{LO}}$ & $6.66_{-1.87-0.27}^{+2.95+0.34}$ & $122_{-32-7}^{+49+6}$ & $305_{-76-16}^{+112+14}$ & $681_{-159-34}^{+228+26}$ \\
\hline$\sigma_{\mathrm{NLL}}$ & $5.20_{-0.36-0.19}^{+0.40+0.29}$ & $103_{-14-5}^{+17+5}$ & $253_{-36-10}^{+44+10}$ & $543_{-88}^{+101+18}$ \\
$\sigma_{\mathrm{NLO}}$ leading & $6.42_{-0.76-0.23}^{+0.42+0.35}$ & $152_{-15-8}^{+7}+8$ & $381_{-32-17}^{+12+16}$ & $835_{-60}^{+18+29}$ \\
$\sigma_{\mathrm{NLO}}$ & $6.72_{-0.76-0.24}^{+0.36+0.37}$ & $159_{-21-9}^{+20+8}$ & $402_{-51-18}^{+49+17}$ & $889_{-106-32}^{+107+31}$ \\
\hline$\sigma_{\mathrm{NLO}+\mathrm{NNLL}}$ & $6.48_{-0.21-0.25}^{+0.17+0.32}$ & $146_{-7-8}^{+7+8}$ & $368_{-14-15}^{+20+19}$ & $813_{-36-35}^{+50+30}$ \\
$\sigma_{\mathrm{NNLO}, \text { approx }}$ (scheme A) & $6.72_{-0.47-0.24}^{+0.45+0.33}$ & $162_{-14-9}^{+19+9}$ & $411_{-35_{-20}^{+17}}^{+49+17}$ & $911_{-77-32}^{+111+35}$ \\
$\sigma_{\mathrm{NNLO}, \text { approx }}$ (scheme B) & $6.55_{-0.41-0.24}^{+0.32+0.33}$ & $149_{-9}^{+10+8}$ & $377_{-23-18}^{+28+16}$ & $832_{-50-29}^{+65+31}$ \\
\hline
\end{tabular}

Table 4: Same as Table 3, but with the "educated" scale choice $\mu_{f}=m_{t}$.

10-15\% enhancement over the fixed-order NLO result. A more important effect is that the resummation stabilizes the scale dependence significantly. Concerning the approximate NNLO schemes, the results from scheme A are noticeably higher than those from scheme B, but these differences are well inside the quoted errors. Since the two schemes differ only by terms proportional to $\delta(1-z)$, this gives an indication of the size of the unknown constant terms.

To some extent, the enhancement effect resulting from the resummation of the leading threshold terms can be mimicked using fixed-order results evaluated at a significantly lower factorization and renormalization scale $\mu_{f}$. Such an "educated" scale choice, which is often adopted in the literature on fixed-order calculations, is $\mu_{f}=m_{t}$. Table 4 shows the crosssection predictions obtained in this case. The fixed-order results are indeed significantly enhanced with this scale choice. The resummed predictions, on the other hand, do not change much compared to those shown in Table 3. 


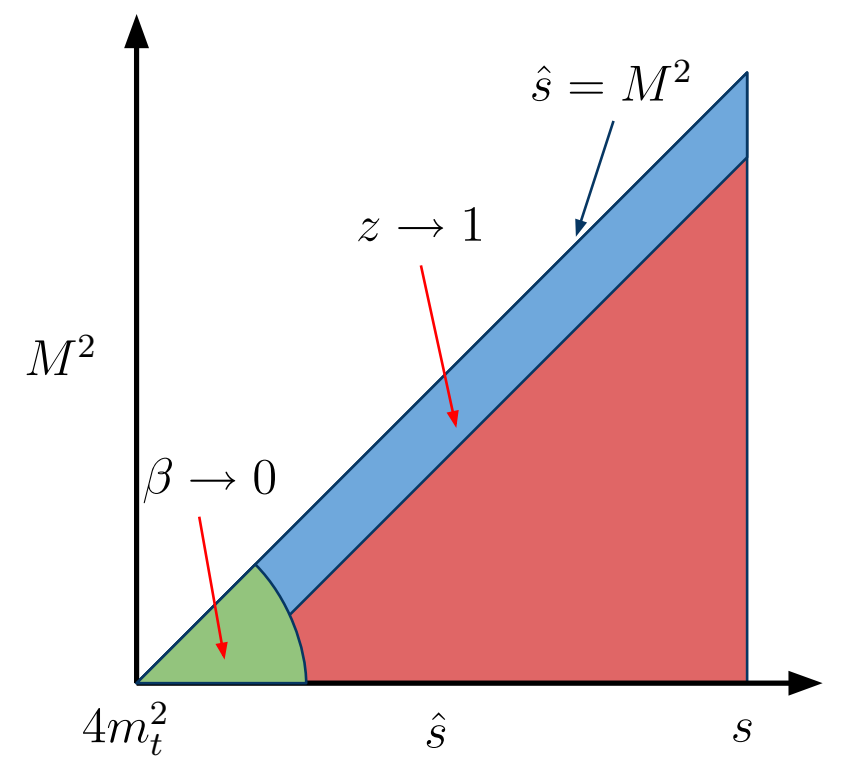

Figure 13: Phase space in the $\left(\hat{s}, M^{2}\right)$ plane. In the blue region along the diagonal threshold singularities arise, and the cross sections receives its main contributions. In the small green region near the origin Coulomb singularities appear and the small- $\beta$ expansion applies.

\subsection{Total cross section: Comparison with previous calculations}

\subsubsection{Small- $\beta$ expansion}

The approach pursued here offers an alternative to the direct threshold expansion of the total partonic cross section in the limit $\beta \rightarrow 0$, corresponding to $\hat{s} \rightarrow 4 m_{t}^{2}$. In this case not only the phase-space for real gluon emissions shrinks to zero, but in addition the top and antitop quarks are produced at rest in the partonic center-of-mass frame, which implies that in addition to soft-gluon singularities one encounters Coulomb singularities. The leading terms in the $\beta \rightarrow 0$ limit were first calculated at NNLO in [40,42], and later corrected in [45].

It is important to emphasize that the leading singular contributions to the total cross section arising from the $\beta \rightarrow 0$ limit do not coincide with those arising from the limit $z \rightarrow 1$, even after integrating over all kinematic variables. The reason is that after convolution with the PDFs there are no truly small scale ratios left in the process (the total center-of-mass energy $\sqrt{s}$ at the Tevatron or LHC are so large that they can be taken to infinity compared with the scale $m_{t}$ ). The large perturbative corrections to the cross section arise dynamically, because of the relatively strong fall-off of the parton luminosities combined with the fact that the partonic cross sections receive their dominant contributions from the region near Bornlevel kinematics $[64,66]$. One would then expect that the most accurate account of enhanced perturbative corrections should be the one that captures enhanced contributions in all relevant regions of phase space.

In terms of the variables $\hat{s}$ and $M^{2}$, the phase space is given by the triangular region $4 m_{t}^{2} \leq M^{2} \leq \hat{s} \leq s$, as illustrated in Figure 13. The large threshold terms considered in the present paper and in our previous work [56] are located along the diagonal, where the 
partonic cross sections are largest. The large corrections arising in the $\beta \rightarrow 0$ limit are located near the origin of the diagram, where both $\hat{s}$ and $M^{2}$ approach $4 m_{t}^{2}$. The parton luminosities are largest for small values of $\hat{s}$. It is obvious from the figure, and also by considering the invariant mass distributions shown in Figure 8, that the region near the origin gives only a very small contribution to the total cross section. This fact is most clearly demonstrated by the distributions in the variable $\beta_{t}$ shown in Figure 11, which peak at $\beta_{t} \approx 0.6$ (Tevatron) and $\beta_{t} \approx 0.7$ (LHC). The region of small velocity, say below $\beta_{t}=0.2$, obviously yields very small contributions to the total cross sections. Since the variable $\beta$ is always larger than $\beta_{t}$, this conclusion is even more true for the small- $\beta$ region. On the contrary, the approach pursued in the present work accounts for enhanced perturbative contributions in all regions of phase space giving rise to large contributions to the total cross section. It is completely analogous to threshold (or soft-gluon) resummation for Drell-Yan or Higgs production at fixed value of the lepton pair or Higgs boson mass. Even though one can never be sure how accurately the full NNLO correction to a cross sections is approximated by a subset of calculable terms, we strongly believe that our treatment provides an approximation that captures more physics than that based on the $\beta \rightarrow 0$ limit. We therefore expect our predictions for the invariant mass distribution, total cross section, and forward-backward asymmetry to be the most reliable available at present.

Having just argued that the $\beta \rightarrow 0$ limit is not of much relevance for the total cross section, it is nevertheless interesting to study how well our predictions fare in this region. Since in our case the top quarks are generically not at rest in their center-of-mass frame, we are not dealing with Coulomb singularities, and hence our approximate prediction for the NNLO corrections to the cross section misses a subset of terms involving potential-gluon exchange. We will now study in more detail which of the singular terms in the $\beta \rightarrow 0$ limit can be recovered in our approach. To this end, we write the total cross section in the form

$$
\sigma\left(s, m_{t}^{2}\right)=\frac{\alpha_{s}^{2}}{m_{t}^{2}} \sum_{i j} \int_{4 m_{t}^{2}}^{s} \frac{d \hat{s}}{s} f f_{i j}\left(\frac{\hat{s}}{s}, \mu\right) f_{i j}\left(\frac{4 m_{t}^{2}}{\hat{s}}, \mu\right) .
$$

We can obtain an expression for the perturbative functions $f_{i j}$ by integrating (5) over all of phase-space, in which case we find

$$
\alpha_{s}^{2} f_{i j}\left(\frac{4 m_{t}^{2}}{\hat{s}}, \mu\right)=\frac{8 \pi m_{t}^{2}}{3 \hat{s}} \int_{2 m_{t}}^{\sqrt{\hat{s}}} \frac{d M}{M} \int_{-1}^{1} d \cos \theta \sqrt{1-\frac{4 m_{t}^{2}}{M^{2}}} C_{i j}\left(\frac{M^{2}}{\hat{s}}, M, m_{t}, \cos \theta, \mu\right) .
$$

We can now evaluate the above formula in the limit $\hat{s} \rightarrow 4 m_{t}^{2}$. Defining expansion coefficients for the functions $f_{i j}$ as

$$
f_{i j}=f_{i j}^{(0)}+4 \pi \alpha_{s} f_{i j}^{(1)}+\left(4 \pi \alpha_{s}\right)^{2}\left[f_{i j}^{(2,0)}+f_{i j}^{(2,1)} \ln \left(\frac{\mu_{f}^{2}}{m_{t}^{2}}\right)+f_{i j}^{(2,2)} \ln ^{2}\left(\frac{\mu_{f}^{2}}{m_{t}^{2}}\right)\right]+\ldots,
$$

the answer for the scale-independent pieces with $n_{h}=1, n_{l}=5$, and $N=3$ can be written as

$$
f_{q \bar{q}}^{(2,0)}=\frac{1}{\left(16 \pi^{2}\right)^{2}} \frac{\pi \beta}{9}\left[910.22 \ln ^{4} \beta-1315.5 \ln ^{3} \beta+592.29 \ln ^{2} \beta+452.52 \ln \beta\right.
$$




\begin{tabular}{|c|c|c|c|c|}
\hline & Tevatron & $\mathrm{LHC}(7 \mathrm{TeV})$ & LHC $(10 \mathrm{TeV})$ & $\mathrm{LHC}(14 \mathrm{TeV})$ \\
\hline$\sigma_{\mathrm{NLO}}$ & $5.79_{-0.80-0.22}^{+0.79+0.33}$ & $133_{-19-7}^{+21+7}$ & $341_{-46-15}^{+50+14}$ & $761_{-101-27}^{+105+26}$ \\
\hline$\sigma_{\mathrm{NLO}}$, leading & $5.49_{-0.78-0.20}^{+0.78+0.31}$ & $134_{-17-7}^{+16+7}$ & $341_{-38-14}^{+34+14}$ & $761_{-75-26}^{+64+25}$ \\
\hline$\sigma_{\mathrm{NLO},} \beta$-exp. v1 & $8.22_{-0.88-0.33}^{+0.54+0.49}$ & $157_{-16-8}^{+12+8}$ & $395_{-36-15}^{+24+14}$ & $877_{-73-30}^{+49+29}$ \\
\hline$\sigma_{\mathrm{NLO},} \beta$-exp. v2 & $6.59_{-0.95-0.25}^{+0.96+0.38}$ & $151_{-18-8}^{+15+8}$ & $386_{-39-16}^{+30+15}$ & $863_{-73-30}^{+49+29}$ \\
\hline$\sigma_{\mathrm{NLO}+\mathrm{NNLL}}$ & $6.30_{-0.19-0.23}^{+0.19+0.31}$ & $149_{-7-8}^{+7+8}$ & $373_{-15-16}^{+17+16}$ & $821_{-42-31}^{+40+24}$ \\
\hline$\sigma_{\mathrm{NNLO},} \beta$-exp. v1 & $7.37_{-0.20-0.29}^{+0.01+0.39}$ & $156_{-5-8}^{+2+8}$ & $392_{-11-17}^{+4}$ & $865_{-17-30}^{+5}$ \\
\hline$\sigma_{\mathrm{NNLO}}, \beta$-exp.+potential v1 & $7.30_{-0.18-0.28}^{+0.01+0.39}$ & $158_{-6-8}^{+3+8}$ & $398_{-13-17}^{+7}$ & $880_{-22-31}^{+12+29}$ \\
\hline$\sigma_{\mathrm{NNLO},} \beta$-exp. v2 & $6.98_{-0.40-0.27}^{+0.17+0.37}$ & $156_{-6-8}^{+2+8}$ & $394_{-10-17}^{+2}$ & $871_{-14-31}^{+0}+29$ \\
\hline$\sigma_{\mathrm{NNLO}}, \beta$-exp. + potential v2 & $6.95_{-0.39-0.26}^{+0.16+0.36}$ & $159_{-7-8}^{+3+8}$ & $401_{-12-17}^{+6}$ & $888_{-19-32}^{+7}$ \\
\hline
\end{tabular}

Table 5: Results for the total cross section in pb, using the default choice $\mu_{f}=400 \mathrm{GeV}$. Some numbers from Table 3 are compared with results obtained from different implementations of the small- $\beta$ expansion (see text for explanation). The errors have the same meaning as before.

$$
\begin{aligned}
& \left.-\frac{1}{\beta}\left(140.37 \ln ^{2} \beta+18.339 \ln \beta-72.225\right)+f_{q \bar{q}}^{\text {potential }}\right]+\ldots, \\
f_{g g}^{(2,0)}= & \frac{1}{\left(16 \pi^{2}\right)^{2}} \frac{7 \pi \beta}{192}\left[4608.0 \ln ^{4} \beta-1894.9 \ln ^{3} \beta-912.35 \ln ^{2} \beta+2747.5 \ln \beta\right. \\
& \left.+\frac{1}{\beta}\left(496.30 \ln ^{2} \beta+400.41 \ln \beta-236.22\right)+f_{g g}^{\text {potential }}\right]+\ldots,
\end{aligned}
$$

where the dots refer to $\mathcal{O}\left(\beta \ln ^{0} \beta\right)$ terms, which are yet unknown. We have split the answer into the piece recovered from the expansion of our results, which we have written explicitly, and a piece related to NNLO effects from potential gluons, which would be recovered from the small- $\beta$ expansion of the as yet unknown $\mu$-independent part of the NNLO coefficient $C_{0}$ in (99). Such potential-gluon contributions were obtained in [45] by using the two-loop calculations of [89-91], and lead to the additional terms

$$
\begin{aligned}
& f_{q \bar{q}}^{\text {potential }}=\frac{3.6077}{\beta^{2}}+\frac{1}{\beta}(50.445 \ln \beta-68.274)+76.033 \ln \beta, \\
& f_{g g}^{\text {potential }}=\frac{68.547}{\beta^{2}}+\frac{1}{\beta}(-79.270 \ln \beta+227.59)-290.76 \ln \beta .
\end{aligned}
$$

The coefficients $f_{i j}^{(2,1)}$ and $f_{i j}^{(2,2)}$ (with $i j=q \bar{q}, g g$ ) in (109) are also recovered from the expansion of our results, up to terms of $\mathcal{O}\left(\beta^{2}\right)$. We have checked that they agree with the results given in [42] when expanded to that order.

It is worth noting that after obtaining the small- $\beta$ expansion as in (110), one can replace the approximated Born prefactors, $\pi \beta / 9$ in the $q \bar{q}$ case and $7 \pi \beta / 192$ in the $g g$ case, with the exact Born-level results. This procedure has been adopted in the recent literature on the 
small- $\beta$ expansion $[40,42,45]$. Therefore, we will differentiate the two versions of the small- $\beta$ expansion: version 1 refers to the version where a complete expansion is carried out as in (110), while version 2 refers to the version with the exact Born-level results as prefactors.

We are now ready to compare our results to those obtained using the small- $\beta$ expansion. We focus first on a comparison at NLO, where the exact answers are known. In the upper portion of Table 5, we show the results of the different approximations as well as the exact NLO cross sections. As we have mentioned in the last section, our NLO leading singular terms are always a good approximation to the exact NLO results, at both the Tevatron and the LHC. On the other hand, the small- $\beta$ expansion version 1 tends to overestimate the cross section at the Tevatron by more than $2 \mathrm{pb}$. By incorporating the exact Born prefactors (version 2) the small- $\beta$ expansion works better, but still it overestimates the exact results by about $1 \mathrm{pb}$. At the LHC, the small- $\beta$ expansion happens to give results closer to the exact answers. However, as we will now explain, this is a coincidence. In Figure 14 we plot the NLO corrections to the cross sections, including the parton luminosities, as functions of $\beta$. The black solid curves show the exact results, the red solid curves our leading singular terms, and the dotted curves the results obtained using version 2 of the small- $\beta$ expansion (the dashed curves will be explained in the next subsection). In the small- $\beta$ region, all the approximations work rather well as expected. With increasing $\beta$, the different approximations start to deviate from one another. We observe that, at both the Tevatron and the LHC, our approximations always reproduce the shapes of the exact results quite well, which is not at all achieved by the small- $\beta$ expansion. The fact that the small- $\beta$ expansion overestimates the cross section at the Tevatron, where the $q \bar{q}$ channel dominates, is evident from the left plots in Figure 14. At the LHC, where the $g g$ channel dominates, the small- $\beta$ expansion does not reproduce the shapes of the exact results, even though it happens that the integrated cross sections are close to the exact ones due to a coincidental cancellation. However, it is unlikely that a similar cancellation will happen at NNLO.

We next compare our best prediction, NLO+NNLL, to the best prediction obtained using the small- $\beta$ expansion at NNLO, which is obtained by adding the approximate NNLO corrections derived using the small- $\beta$ expansion to the exact NLO cross sections. Without knowing the exact expression for the NNLO corrections, it is hard to tell which one is closer to the true answer, but we can study the validity of small- $\beta$ expansion by investigating the effects of the subleading terms in $\beta$ that are contained in our results. We have included in Table 5 the numerical results for the cross section obtained by evaluating the small- $\beta$ expansion (110) of our approximate NNLO formula, without including the extra potential terms (labeled " $\beta$ exp." in the table), and that obtained using this expansion plus the potential terms in (111) (labeled " $\beta$-exp.+potential"). We notice that the NLO+NNLL resummed results and the small- $\beta$ expansion differ by about $10-15 \%$ at the Tevatron and about $6 \%$ at the LHC. On the other hand, the effect of adding the potential-gluon contributions to the small- $\beta$ expansion, which cannot be reproduced in our approach, is always smaller than $2 \%$. We conclude that the bulk of the terms that become singular in the $\beta \rightarrow 0$ limit are accounted for in our approach. The reverse statement is not true. A resummation based on the $\beta \rightarrow 0$ expansion does not account for the bulk of the terms that become singular in the $z \rightarrow 1$ limit, and our analysis suggests that subleading terms in $\beta$ are by no means generically small.

The CDF collaboration has recently published a combined value for the $t \bar{t}$ cross section, 

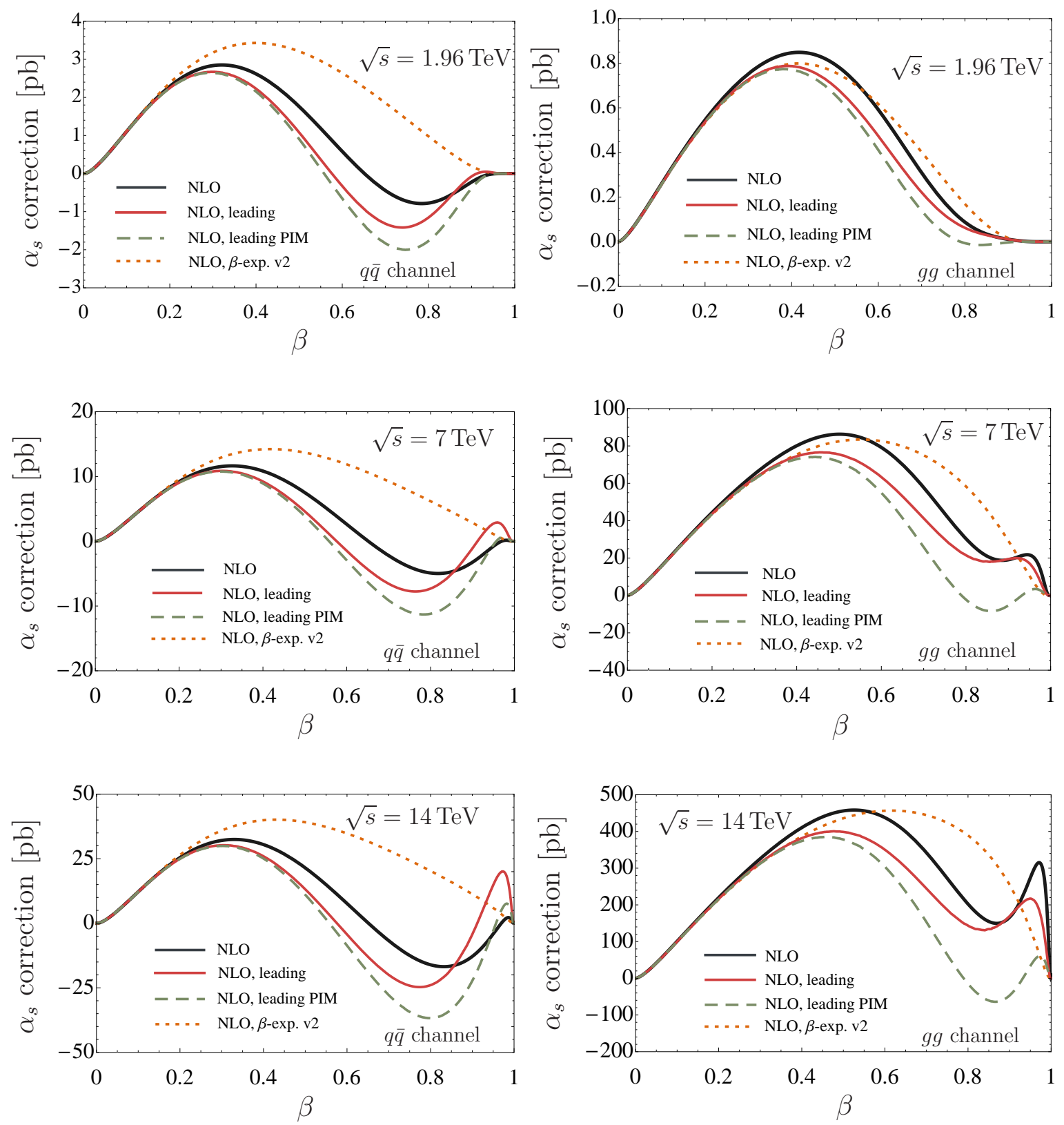

Figure 14: The $\alpha_{s}$ corrections to $d \sigma / d \beta$ for the different approximations mentioned in the text at the Tevatron and LHC, with $\mu_{f}=m_{t}$. The plots on the left side show the $q \bar{q}$ channel, those on the right the $g g$ channel.

finding $\sigma_{\exp }=(7.50 \pm 0.48) \mathrm{pb}[92]$. They have compared their result with the theoretical prediction $\sigma_{\mathrm{th}}=\left(7.46_{-0.80}^{+0.66}\right) \mathrm{pb}$ derived using the small- $\beta$ expansion approach from [42]. This number is significantly higher than our best prediction $\sigma_{\mathrm{NLO}+\mathrm{NNLL}}=\left(6.30_{-0.19-0.23}^{+0.19+0.31}\right)$ pb given in Table 3 , but it is also higher than the value $\sigma_{\mathrm{NNLO}} \beta$-exp.+potential v2 $=\left(6.95_{-0.39-0.26}^{+0.16+0.36}\right)$ pb shown in Table 5, which is our own result obtained using the small- $\beta$ expansion. This latter difference is partly due to a smaller top-quark mass $(172.5 \mathrm{GeV}$ instead of $173.1 \mathrm{GeV})$, a different PDF 
set (CTEQ6.6 instead of MSTW2008NNLO), and a different value of $\alpha_{s}\left(M_{Z}\right)$ (0.118 instead of 0.117 ), and partly due to a lower factorization scale ( $m_{t}$ instead of $400 \mathrm{GeV}$ ). Changing our input values to those used in [42], we would obtain $\sigma_{\mathrm{NLO}+\mathrm{NNLL}}=\left(6.86_{-0.20-0.35}^{+0.19+0.44}\right) \mathrm{pb}$ and $\sigma_{\mathrm{NNLO}}, \beta$-exp.+potential v2 $=\left(7.55_{-0.27-0.38}^{+0.01+0.49}\right) \mathrm{pb}$. We observe that with these settings the predictions are indeed higher, ${ }^{2}$ and our $\sigma_{\mathrm{NNLO}} \beta$-exp.+potential v2 is now very close to the result from [42]. The remaining slight difference can be attributed to the use of an incorrect two-loop coefficient in [42], which was later corrected in [45]. Our best prediction $\sigma_{\mathrm{NLO}+\text { NNLL }}$ is still about $10 \%$ lower than the result obtained using the small- $\beta$ expansion, which can be understood from the discussions in the previous paragraphs, but is consistent with the experimental result within errors.

\subsubsection{Other approaches and kinematics}

Since in this paper we are interested in the invariant mass distribution of the $t \bar{t}$ pair, we have adopted the so-called pair-invariant mass (PIM) kinematics, which is defined by

$$
N_{1}\left(P_{1}\right)+N_{2}\left(P_{2}\right) \rightarrow t \bar{t}\left(p_{3}+p_{4}\right)+X(k) .
$$

When calculating the transverse-momentum and rapidity distributions of the top quark, with the momentum of the anti-top quark integrated over, one instead considers the one-particle inclusive (1PI) kinematics, which can be written as

$$
N_{1}\left(P_{1}\right)+N_{2}\left(P_{2}\right) \rightarrow t\left(p_{3}\right)+X^{\prime}[\bar{t}]\left(p_{4}^{\prime}\right),
$$

where here the final state $X^{\prime}$ contains the $\bar{t}$ quark accompanied by additional emissions (see e.g. $[50,51])$. In both cases, the threshold limit corresponds to the limit in which these extra emissions are soft, implying $k^{0} \rightarrow 0$ and $p_{4}^{\prime 2} \rightarrow m_{t}^{2}$, respectively. In the PIM case, this implies $M^{2} \rightarrow \hat{s}$, where $M^{2}=\left(p_{3}+p_{4}\right)^{2}$ and $\hat{s}=\left(p_{1}+p_{2}\right)^{2}$ can be calculated without reference to the unobserved momentum $k$. In the 1 PI case, it implies $s_{4} / \hat{s}=\left(1-2 E_{3} / \sqrt{\hat{s}}\right) \rightarrow 0$, where $s_{4}=\hat{s}+t_{1}+u_{1}$ can be calculated from (3) without reference to the unobserved momentum $p_{4}^{\prime}$, and $E_{3}$ denotes the energy of the top quark in the partonic center-of-mass frame.

Although the PIM and 1PI kinematics are applicable in different differential distributions, they can both be integrated over to obtain the total cross section. In the sense that they are both applicable in the limit where the extra emissions are soft, threshold resummation for the total cross section based on PIM or 1PI kinematics amounts to resumming the same leading contributions, but differs by subleading corrections. Several authors have found significant numerical differences between the results obtained using the two kinematic schemes (see e.g. [50-52]). Therefore, it is interesting to work out the 1PI kinematics also in our approach.

Before moving onto 1PI kinematics, it is however necessary to point out an important difference between our results and previous ones obtained using PIM kinematics. In the traditional approach, the leading singular terms in $(1-z)$ are written in terms of the distributions

\footnotetext{
${ }^{2}$ The difference between the central values of our NLO+NNLL predictions (6.86 pb vs. $\left.6.30 \mathrm{pb}\right)$ is $0.56 \mathrm{pb}$, of which about $0.18 \mathrm{pb}$ is due to the scale choice, about $0.10 \mathrm{pb}$ is due to the smaller $m_{t}$, and another $0.28 \mathrm{pb}$ is due to the different PDF set and $\alpha_{s}\left(M_{Z}\right)$ value.
} 
$P_{n}(z)=\left[\ln ^{n}(1-z) /(1-z)\right]_{+}$, while in our approach they are more naturally written in terms of the distributions

$$
P_{n}^{\prime}(z)=\left[\frac{1}{1-z} \ln ^{n}\left(\frac{M^{2}(1-z)^{2}}{\mu^{2} z}\right)\right]_{+} .
$$

The additional factor of $z$ in the logarithms is a subleading effect, but it is relevant in practice. This has been studied in detail for the simpler cases of Drell-Yan [64] and Higgs production [66], but analogous remarks hold also in the present case. Of crucial importance in this context is the fact that our resummation method works directly in momentum space [62], and as a result the matching onto analytical fixed-order expressions is particularly transparent. We have observed in previous work $[64,66]$ that at NLO in perturbation theory all logarithmic corrections to Drell-Yan and Higgs production, even those entering at subleading orders in $(1-z)$, can be expressed in terms of the "hard" and "soft" logarithms $\ln \left(M^{2} / \mu_{f}^{2}\right)$ and $\ln \left(\hat{s}(1-z)^{2} / \mu_{f}^{2}\right)$, where $M^{2}=M_{l^{+} l^{-}}^{2}$ or $m_{H}^{2}$ as appropriate, and $\hat{s}=M^{2} / z$. Choosing $\hat{s}(1-z)^{2}=M^{2}(1-z)^{2} / z$ as the argument of the soft functions in the SCET approach thus provides an "optimal" matching onto fixed-order expressions, which automatically accounts for subleading logarithms $\ln z$. We have observed that, with this choice, the leading singular terms near partonic threshold account for the bulk of the NLO and NNLO corrections to the Drell-Yan and Higgs production cross sections. In the present paper, we have shown that the same is true for the NLO corrections to the $t \bar{t}$ production cross sections at the Tevatron and LHC. Our resummation procedure is thus different, in the way we treat subleading terms, from the traditional PIM scheme, and for the reason just explained it is bound to be more accurate. This can be clearly seen from Figure 14, where we have plotted the NLO leading singular terms both in our scheme (red solid curves) and in the traditional PIM scheme (dashed curves). One can find that our results always provide a better approximation to the exact NLO correction than the traditional PIM results. Especially for the $g g$ channel at the LHC, where the traditional PIM results were argued to be a bad approximation [51,52], our result still provides a decent approximation.

We now turn to the 1PI kinematics, in which case the relevant differential cross section is

$$
\frac{d^{2} \sigma}{d T_{1} d U_{1}}=\frac{8 \pi}{3 s^{2}} \sum_{i, j} \int \frac{d x_{1}}{x_{1}} \frac{d x_{2}}{x_{2}} f_{i / N_{1}}\left(x_{1}, \mu_{f}\right) f_{j / N_{2}}\left(x_{2}, \mu_{f}\right) C_{i j}^{\prime}\left(s_{4}, t_{1}, u_{1}, m_{t}, \mu_{f}\right) .
$$

Here $T_{1}=\left(P_{1}-p_{3}\right)^{2}-m_{t}^{2}$ and $U_{1}=\left(P_{2}-p_{3}\right)^{2}-m_{t}^{2}$ are the hadronic counterparts of $t_{1}$ and $u_{1}$. Near the partonic threshold $s_{4} \rightarrow 0$, the hard-scattering kernels $C_{i j}^{\prime}$ can again be factorized as

$$
C_{i j}^{\prime}\left(s_{4}, t_{1}, u_{1}, m_{t}, \mu_{f}\right)=\operatorname{Tr}\left[\boldsymbol{H}_{i j}\left(t_{1}, u_{1}, m_{t}, \mu_{f}\right) \boldsymbol{S}_{i j}^{\prime}\left(s_{4}, t_{1}, u_{1}, m_{t}, \mu_{f}\right)\right],
$$

where the hard functions $\boldsymbol{H}_{i j}$ are the same as in the PIM case, once we re-express $M^{2}$ and $\cos \theta$ in terms of $t_{1}$ and $u_{1}$. The new soft functions $\boldsymbol{S}_{i j}^{\prime}$ can be calculated from (68) with the replacement $k^{0} \rightarrow v_{4} \cdot k$. After these changes, all techniques we have developed in this paper can be applied to the 1PI case, too. In particular, we can derive a resummed expression for the hard-scattering kernels

$$
C^{\prime}\left(s_{4}, t_{1}, u_{1}, m_{t}, \mu_{f}\right)=\exp \left[2 a_{\Gamma}\left(\mu_{s}, \mu_{f}\right) \ln \frac{m_{t}^{2} \mu_{s}^{2}}{t_{1} u_{1}}+4 a_{\gamma^{\phi}}\left(\mu_{s}, \mu_{f}\right)\right]
$$




$$
\begin{aligned}
\times \operatorname{Tr}\left[\boldsymbol{U}\left(t_{1}, u_{1}, m_{t}, \mu_{h}, \mu_{s}\right) \boldsymbol{H}\left(t_{1}, u_{1}, m_{t}, \mu_{h}\right) \boldsymbol{U}^{\dagger}\left(t_{1}, u_{1}, m_{t}, \mu_{h}, \mu_{s}\right)\right. \\
\left.\quad \times \tilde{\boldsymbol{s}}^{\prime}\left(\partial_{\eta}, t_{1}, u_{1}, m_{t}, \mu_{s}\right)\right] \frac{1}{s_{4}}\left(\frac{s_{4}}{m_{t} \mu_{s}}\right)^{2 \eta} \frac{e^{-2 \gamma_{E} \eta}}{\Gamma(2 \eta)}
\end{aligned}
$$

By setting $\mu_{h}=\mu_{s}=\mu_{f}$ in the above formula, we can derive the leading singular terms in 1PI kinematics. We have checked that the leading terms at NLO agree with those in the literature [14,59]. A numerical analysis of the resummation in 1PI kinematics is better to be done in the context of transverse-momentum and rapidity distributions of the top quark, where a systematic study as we have done for PIM kinematics can be carried out to determine the optimal choices of the scales $\mu_{h}, \mu_{s}$ and $\mu_{f}$. Therefore we will leave it for a future analysis. Nevertheless, given the arguments in the previous paragraphs, we do not expect significant differences in the results for the total cross sections obtained using the two kinematic schemes.

\subsection{Charge and forward-backward asymmetries}

The top-quark pair charge asymmetry is an important observable at the Tevatron, which originates from the difference in the production rates for top and anti-top quarks at fixed scattering angle or rapidity $[20,21]$. To describe the total charge asymmetry, we first introduce a charge-asymmetric cross section as the difference between the production of top and anti-top quarks in the forward direction,

$$
\Delta \sigma_{c} \equiv \int_{0}^{1} d \cos \theta \int_{2 m_{t}}^{\sqrt{s}} d M\left[\frac{d^{2} \sigma^{p \bar{p} \rightarrow t \bar{t} X}}{d M d \cos \theta}-\frac{d^{2} \sigma^{p \bar{p} \rightarrow \bar{t} t X}}{d M d \cos \theta}\right]
$$

The total charge asymmetry is then defined by $A_{c}=\Delta \sigma_{c} / \sigma$, where $\sigma$ is the total cross section. As a consequence of the charge conjugation invariance of QCD, $\Delta \sigma_{c}$ is the same as the difference between the cross sections for the production of top quarks in the forward and backward directions,

$$
\Delta \sigma_{c}=\Delta \sigma_{\mathrm{FB}} \equiv \int_{2 m_{t}}^{\sqrt{s}} d M\left[\int_{0}^{1} d \cos \theta \frac{d^{2} \sigma^{p \bar{p} \rightarrow t \bar{t} X}}{d M d \cos \theta}-\int_{-1}^{0} d \cos \theta \frac{d^{2} \sigma^{p \bar{p} \rightarrow t \bar{t} X}}{d M d \cos \theta}\right]
$$

Therefore, the charge asymmetry can also be interpreted as a forward-backward asymmetry for the top quark, defined as $A_{\mathrm{FB}}^{t}=\Delta \sigma_{\mathrm{FB}} / \sigma$.

At leading order in $\mathrm{QCD}\left(\mathcal{O}\left(\alpha_{s}^{2}\right)\right)$, the charge-asymmetric cross section $\Delta \sigma_{c}$ vanishes. This quantity receives non-zero contributions starting at $\mathcal{O}\left(\alpha_{s}^{3}\right)$. These arise if, in the interference of one-loop and tree-level diagrams, the top-quark fermionic line and the light-quark fermionic line are connected by three gluons. The same observation applies also to the interference of two tree-level diagrams with three particles in the final state. In Figure 15 we show the interference of the planar box with the tree-level diagram and the corresponding interference of real emission diagrams. The other contribution to the asymmetry at $\mathcal{O}\left(\alpha_{s}^{3}\right)$ in the quarkannihilation channel originates from the interference of the crossed box and tree-level diagram 


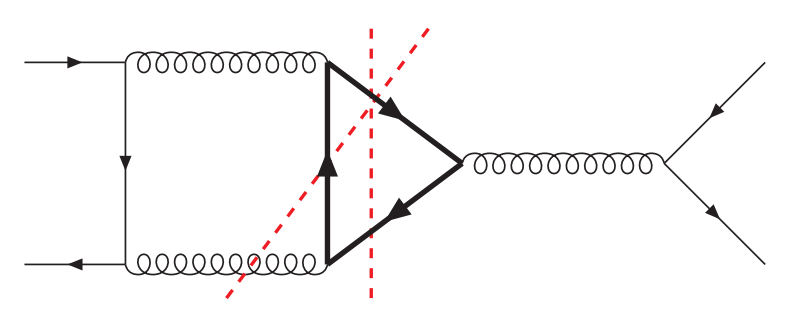

Figure 15: Examples of interferences contributing to the charge-asymmetric cross section. The two-particle cut corresponds to the interference of a one-loop box with the tree-level diagram, while the three-particle cut corresponds to the interference of tree-level diagrams with a $t \bar{t} g$ final state.

(or from the corresponding real emission case). This can be visualized by imagining to cross the two gluons on the left side of the heavy-quark triangle in Figure 15. The color factors multiplying the structure in Figure 15 or its crossed counterpart are respectively

$$
\mathcal{C}_{\text {planar }}=\frac{1}{16 N^{2}}\left(f_{a b c}^{2}+d_{a b c}^{2}\right), \quad \mathcal{C}_{\text {crossed }}=\frac{1}{16 N^{2}}\left(d_{a b c}^{2}-f_{a b c}^{2}\right),
$$

where $f_{a b c}^{2}=\left(N^{2}-1\right) N$ and $d_{a b c}^{2}=\left(N^{2}-1\right)\left(N^{2}-4\right) / N$. When the color factors are stripped off, the interference in Figure 15 and its crossed counterpart satisfy the relation

$$
d \sigma_{\text {planar }}^{p \bar{p} \rightarrow t \bar{t} X}=-d \sigma_{\text {crossed }}^{p \bar{p} \rightarrow \bar{t} X} .
$$

This relation holds both for the three-particle and the two-particle cuts. Therefore, the chargeasymmetric cross section is proportional to $d_{a b c}^{2}$. The interference of the one-loop box diagrams with the tree-level diagram gives a positive contribution to the asymmetry, which is partially canceled by the asymmetry originating from the interference of initial- and final-state radiation diagrams. An additional small contribution to the asymmetry at $\mathcal{O}\left(\alpha_{s}^{3}\right)$ originates from the flavor excitation channel $g q(\bar{q}) \rightarrow t \bar{t} X$ at tree level. The gluon-fusion channel does not contribute to the charge-asymmetric cross section. The study of the charge-asymmetric cross section at $\mathcal{O}\left(\alpha_{s}^{3}\right)$ shows that top quarks (anti-top quarks) are preferably emitted of the direction of the incoming quark (anti-quark); consequently, in $p \bar{p}$ collisions top quarks are preferably emitted in the direction of the incoming proton [20,21].

In the asymmetric cross section (118), the definition of the scattering angle $\theta$ depends on the reference frame, therefore the value of the asymmetry is also frame-dependent. Experimentally the forward-backward asymmetry has been measured both in the laboratory frame and in the $t \bar{t}$ rest frame $[10,11]$, where the result in the former frame is about $70 \%$ of the result in the latter. Near the partonic threshold the $t \bar{t}$ rest frame and the partonic center-of-mass frame coincide. Using the complete $\mathcal{O}\left(\alpha_{s}^{3}\right)$ expressions for $\Delta \sigma_{\mathrm{FB}}$ in [21], we have checked explicitly that the results in these two frames only differ slightly. It is therefore interesting to employ the formulas derived in the previous sections to calculate the forward-backward asymmetry in the partonic center-of-mass frame. The resummation of partonic threshold effects in the forward-backward asymmetry at NLL order in Mellin space was first considered in [49]. In what follows we extend these calculations to NNLL order, and also study the effect of the approximate NNLO corrections in fixed order. 


\begin{tabular}{|l|c|c|c|c|}
\hline \multirow{2}{*}{} & \multicolumn{2}{|c|}{$0.2<\mu_{f} / \mathrm{TeV}<0.8$} & \multicolumn{2}{c|}{$m_{t} / 2<\mu_{f}<2 m_{t}$} \\
\cline { 2 - 5 } & $\Delta \sigma_{\mathrm{FB}}[\mathrm{pb}]$ & $A_{\mathrm{FB}}^{t}[\%]$ & $\Delta \sigma_{\mathrm{FB}}[\mathrm{pb}]$ & $A_{\mathrm{FB}}^{t}[\%]$ \\
\hline NLL & $0.29_{-0.16}^{+0.16}$ & $5.8_{-3.2}^{+3.3}$ & $0.31_{-0.17}^{+0.16}$ & $5.9_{-3.3}^{+3.4}$ \\
NLO, leading & $0.19_{-0.06}^{+0.09}$ & $5.2_{-0.4}^{+0.4}$ & $0.31_{-0.10}^{+0.16}$ & $5.7_{-0.4}^{+0.5}$ \\
NLO & $0.25_{-0.07}^{+0.12}$ & $6.7_{-0.4}^{+0.6}$ & $0.40_{-0.13}^{+0.21}$ & $7.4_{-0.6}^{+0.7}$ \\
\hline NLO+NNLL & $0.40_{-0.06}^{+0.06}$ & $6.6_{-0.5}^{+0.6}$ & $0.45_{-0.07}^{+0.08}$ & $7.3_{-0.7}^{+1.1}$ \\
NNLO, approx (scheme A) & $0.37_{-0.08}^{+0.10}$ & $6.4_{-0.7}^{+0.9}$ & $0.48_{-0.10}^{+0.11}$ & $7.5_{-0.9}^{+1.3}$ \\
NNLO, approx (scheme B) & $0.34_{-0.07}^{+0.08}$ & $5.8_{-0.6}^{+0.8}$ & $0.45_{-0.09}^{+0.09}$ & $6.8_{-0.8}^{+1.1}$ \\
\hline
\end{tabular}

Table 6: The asymmetric cross section and forward-backward asymmetry at the Tevatron, evaluated at different orders in perturbation theory in the partonic center-of-mass frame. The errors refer to perturbative uncertainties related to scale variations, as explained in the text.

The results of our analysis are summarized in Table 6 . In the column labeled $\Delta \sigma_{\mathrm{FB}}$, we show the total charge-asymmetric cross section evaluated at different orders in perturbation theory. As in the previous sections, we have used $m_{t}=173.1 \mathrm{GeV}$ and adapted the order of the PDFs as described in Table 2. For the factorization scale, we consider our standard choice $\mu_{f}=400 \mathrm{GeV}$ as well as $\mu_{f}=m_{t}$. The results in resummed perturbation theory use $\mu_{h}=M$ and $\mu_{s}$ chosen according to (105) by default, and the uncertainties are obtained by varying these up and down by a factor of two and adding the different uncertainties in quadrature. The uncertainties in the fixed-order results are obtained by varying $\mu_{f}$ up and down by a factor of two. The counting used in the table refers to the order at which the differential cross section itself is needed, relative to $\alpha_{s}^{2}$; this differs slightly from the counting in fixed order used in [21], which would count, for instance, our NLO as LO. To obtain the result in fixed order at NLO, we have used the formulas in Appendix A of [21]. At both $\mu_{f}=400 \mathrm{GeV}$ and $\mu_{f}=m_{t}$, the NLO threshold terms recover about $80 \%$ of the full result in fixed order, which is roughly in line with our findings for the NLO corrections to the cross section. The table also includes our results for the forward-backward asymmetry. In calculating the asymmetry, we first evaluate the numerator and denominator of the ratio $A_{\mathrm{FB}}^{t}=\Delta \sigma_{\mathrm{FB}} / \sigma$ to a given order in RG-improved or fixed-order perturbation theory, and then further expand the ratio itself. When performing the calculation in this way, the errors in the asymmetry at NLO are actually smaller than those at NLO+NNLL order, even though the scale variations in the numerator and denominator of the ratio are much larger. We note, however, that if we chose instead to not further expand the ratio, the NLO result would be decreased by about $20 \%$, while the NLO+NNLL result changes only by about $5 \%$ and should therefore be considered the more reliable prediction. In [21], an overall factor of 1.09 due to electroweak corrections is included for the asymmetry. However, a smaller correction was recently obtained in [93], and it is also scale-dependent. Therefore we have chosen not to include these corrections in our results.

Our results are in good agreement with the previous findings reported in $[49,94]$. We have not performed the calculation in the $p \bar{p}$ frame, but expect that the boost to this frame decreases the asymmetry by roughly 30\%, as found in [94]. On the other hand, the CDF 


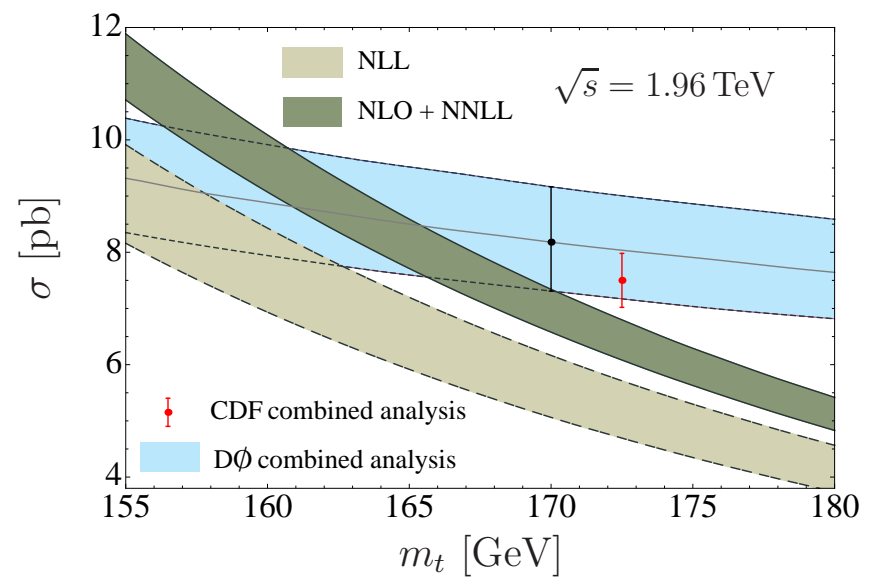

Figure 16: Dependence of the total cross section on the top-quark mass defined in the pole scheme. The NLL and NLO+NNLL bands indicate the scale uncertainties and PDF uncertainties combined linearly, while the blue band represents the dependence of the D0 measurement of the cross section on $m_{t}$, as described in [100]. For comparison, a CDF combined measurement [92] is also shown.

collaboration at the Tevatron recently reported the value $A_{\mathrm{FB}}^{t}(\exp )=(19.3 \pm 6.9) \%$ for the asymmetry in the $p \bar{p}$ frame [12]. The measured asymmetry exceeds the predicted one by about two standard deviations. In light of our results, we conclude that higher-order QCD effects are not sufficient to explain the large experimental value. Possible explanations of this fact in the framework of several new physics scenarios were recently investigated in [94-99].

\subsection{Sensitivity to the top-quark mass}

Up to this point our results were obtained using the default value $m_{t}=173.1 \mathrm{GeV}$ for the top-quark mass defined in the pole scheme. Figure 16 shows the dependence of the total cross section on the value of $m_{t}$. We show our NLL and NLO+NNLL results as bands representing the linearly combined errors from the scale uncertainties and PDF uncertainties, which has been converted to $68 \%$ CL $(1 \sigma)$ to match the confidence level of the experimental errors. We compare our result with a recent combined measurement of the total cross section performed by the D0 collaboration [100]. We also show a combined measurement by the CDF collaboration [92]; however, since they did not provide the mass dependence of the cross section, we cannot use their results to extract the top-quark mass. From the overlap region of the bands we determine the top-quark mass to be $m_{t}=\left(163.0_{-6.3}^{+7.2}\right) \mathrm{GeV}$, which is lower than the latest world average $m_{t}=\left(173.1_{-1.3}^{+1.3}\right) \mathrm{GeV}$ [1]. One should, however, interpret this "discrepancy" with care. It is unclear if the quoted world-average top-quark mass really refers to the pole scheme, and even if it does, to which order of perturbation theory it corresponds. Since the pole mass of a quark is not well-defined beyond perturbation theory, the value of $m_{t}$ in the pole scheme changes significantly in different orders. It would therefore be preferable to perform both the theoretical and experimental analyses with a more physical mass parameter such as that defined in the $\overline{\mathrm{MS}}$ scheme (see below). 

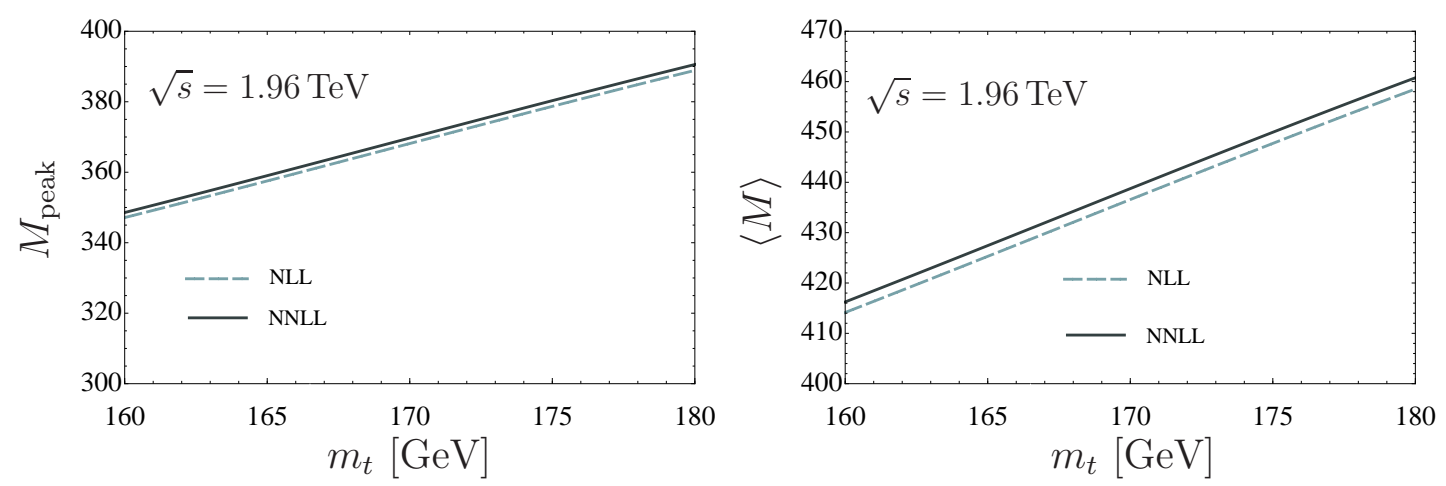

Figure 17: Position of the peak of the invariant mass distribution (left) and of the mean invariant mass $\langle M\rangle$ (right) as functions of the top-quark mass.

\begin{tabular}{|r|r|r|}
\hline & $M_{\text {peak }}[\mathrm{GeV}]$ & $\langle M\rangle[\mathrm{GeV}]$ \\
\hline LO & $375.6_{-0.4}^{+0.5}$ & $450.2_{-1.5}^{+1.7}$ \\
\hline NLL & $374.5_{-0.2}^{+0.2}$ & $443.3_{-0.8}^{+0.6}$ \\
\hline NNLL & $376.1_{-0.2}^{+0.2}$ & $445.5_{-0.3}^{+0.4}$ \\
\hline NLO+NNLL & $375.2_{-0.2}^{+0.3}$ & $445.0_{-0.4}^{+0.4}$ \\
\hline
\end{tabular}

Table 7: Values of the peak position of the mean invariant mass, obtained with $m_{t}=173.1 \mathrm{GeV}$.

As we have seen, there are still rather large uncertainties in using the total cross section as a mean to extract the top-quark mass. We therefore investigate what additional information can be gained from the invariant mass distribution. On the left in Figure 17, we show the position of the peak of the invariant mass distribution, $M_{\text {peak }}$, as a function of $m_{t}$. On the right we show the corresponding dependence of the mean invariant mass $\langle M\rangle$. We do not show the errors arising from scale variations, as they would be too small to be visible on the scales of the plots. As illustrated in Table 7 , both observables turn out to be very stable under the change of the order of perturbation theory. (Observing that the shifts between different orders are larger than those indicated by the scale variations, we however note that scale variation might not be a good estimator of the uncertainty.) They show an almost linear dependence on the mass of the top quark, which leads to a nearly linear translation of errors. A precise measurement of one of these observables would thus lead to a direct measurement of $m_{t}$ with about the same precision. Of course this can only be taken as a qualitative remark at the moment, but with increasing data this might become an important strategy for a precision measurement of $m_{t}$.

It is a well-known fact that the pole mass defined in perturbation theory is an ill-defined concept, as it is plagued by renormalon ambiguities in higher orders of perturbation theory $[101,102]$. While the corresponding intrinsic uncertainty in $m_{t}$ is of order $\Lambda_{\mathrm{QCD}}$ and thus smaller than the present experimental error in this parameter, it is nevertheless useful to consider predictions for the cross section (and distributions) parameterized in terms of a more 


\begin{tabular}{|c|c|}
\hline & $m_{t}[\mathrm{GeV}]$ \\
\hline LO & 164.0 \\
\hline NLO, NLL & 171.7 \\
\hline NNLO, NNLL & 173.1 \\
\hline
\end{tabular}

Table 8: Values of the pole mass $m_{t}$ in different orders of perturbation theory, corresponding to a fixed value $\bar{m}_{t}\left(\bar{m}_{t}\right)=164.0 \mathrm{GeV}$.

suitable mass parameter. For the purpose of illustration, we investigate in the following the impact of using the $\overline{\mathrm{MS}}$ mass $\bar{m}_{t}(\mu)$. We do this by changing the value of the pole mass $m_{t}$ at different orders in perturbation theory, such that the "physical" mass $\bar{m}_{t}(\mu)$ remains the same. This is implemented through the relation

$$
m_{t}=\bar{m}_{t}(\mu)\left[1+\frac{\alpha_{s}(\mu)}{\pi} d^{(1)}+\left(\frac{\alpha_{s}(\mu)}{\pi}\right)^{2} d^{(2)}+\ldots\right],
$$

where for QCD $(N=3)$ with $n_{f}=5$ active, massless flavors [103-105]

$$
d^{(1)}=\frac{4}{3}+L_{m}, \quad d^{(2)}=\frac{2053}{288}+\frac{\pi^{2}}{18}+\frac{\pi^{2}}{9} \ln 2-\frac{\zeta_{3}}{6}+\frac{379}{72} L_{m}+\frac{37}{24} L_{m}^{2},
$$

with $L_{m}=\ln \left(\mu^{2} / \bar{m}_{t}^{2}(\mu)\right)$. We use a fixed input value $\bar{m}_{t} \equiv \bar{m}_{t}\left(\bar{m}_{t}\right)=164.0 \mathrm{GeV}$, chosen such that at two-loop order the corresponding pole-scheme parameter $m_{t}$ in (122) coincides with our default value $m_{t}=173.1 \mathrm{GeV}$. In lower orders we adjust $m_{t}$ such that the $\overline{\mathrm{MS}}$ mass stays unchanged, which leads to the values collected in Table 8. In Figure 18, we show the impact on the invariant mass distribution of choosing $m_{t}$ in the way described above. Compared with Figure 8, we observe an improved convergence of the perturbation theory, both in fixed order and after threshold resummation. This finding, which has previously been made in [42], could have been anticipated by looking at Figure 16 and Table 8 . The increase of the cross section and invariant mass distributions in higher orders is to a large extent compensated by the effect of increasing the value of the pole mass.

\section{Conclusions}

We have studied higher-order perturbative corrections to the differential cross section for $t \bar{t}$ hadroproduction in the partonic threshold region, where the invariant mass of the $t \bar{t}$ pair approaches the partonic center-of-mass energy. This involved using SCET to derive a factorization formula expressing the hard-scattering kernels as products of matrix-valued hard and soft matching coefficients in this kinematic regime, calculating these coefficients to NLO in perturbation theory, and solving the RG equations for these functions directly in momentum space to achieve NNLL accuracy for the resummed differential cross section. We also presented an approximate NNLO formula, which includes all terms proportional to singular 

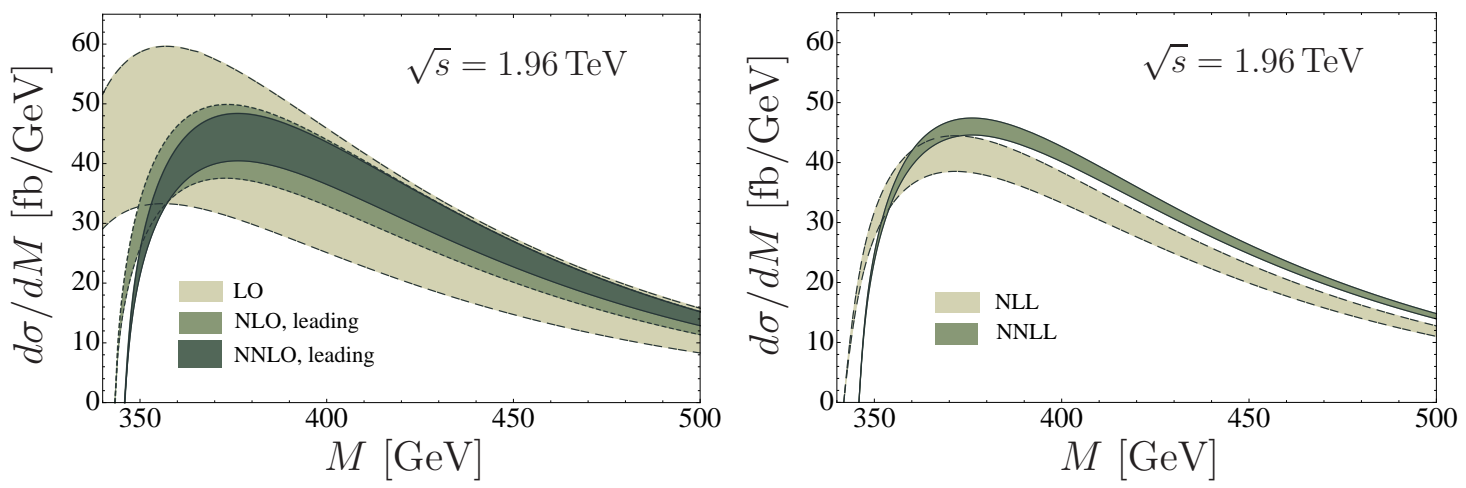

Figure 18: Fixed order (left) and resummed (right) results for $d \sigma / d M$, changing $m_{t}$ at different orders in perturbation theory as shown in Table 8.

plus distributions in the variable $(1-z)$, as well the scale-dependent part of the coefficient of the $\delta(1-z)$ term. The momentum-space resummation techniques allow for a straightforward matching of the resummed results with the exact results at NLO in fixed-order perturbation theory. We used this fact to perform numerical studies of the invariant mass distribution, the total cross section, and the forward backward asymmetry at NLO+NNLL order.

Our results are expected to work progressively better when the invariant mass $M$ becomes large, since in that case the partonic threshold region becomes more important due to kinematics. However, our numerical results show that the full result at NLO in fixed-order perturbation theory is dominated by the leading terms in the threshold expansion, even at low values of invariant mass near the peak of the distribution. This provides evidence for a dynamical enhancement of the partonic threshold region. Assuming this to be the case also at higher orders in perturbation theory, we have argued that our results obtained at NLO+NNLL order provide the best available predictions for the $t \bar{t}$ invariant mass spectrum in the whole range of $M$. The benefits of performing the resummation are best seen through the reduction of scale dependence and the improvement of the convergence shown in Figure 7-10. Compared to the fixed-order NLO results, the RG resummation reduces the scale uncertainties in the differential cross sections and therefore provide the more reliable predictions.

In view of the accuracy we can achieve for the differential cross section, we have also made predictions for the total cross section and the forward-backward asymmetry by integrating the differential distribution over the appropriate regions of phase-space. We believe that these results, summarized in Tables 3, 4 and 6, are currently the best predictions for these observables. In particular, we have compared our results for the total cross section with those obtained in the conventional approach of expanding around $\beta \rightarrow 0$, where the topquark pair is produced nearly at rest. By expanding our approximate NNLO formulas in the limit $\beta \rightarrow 0$ and dropping subleading terms in $\beta$, we recover the results recently obtained in [45], up to terms at NNLO related to potential-gluon exchange. A numerical analysis showed that the contributions of these extra potential-gluon corrections are actually smaller than the subleading terms in $\beta$ contained in our formulas, whose contribution is about $6-15 \%$. This is not surprising, because the cross section receives the dominant contributions from regions with $\beta \sim 0.6$, which is not especially small. For this reason, it seems to us that the 
results obtained here offer a legitimate alternative for including higher-order corrections to the total cross section. As for the forward-backward asymmetry, our results are in quantitative agreement with previous findings, and thus cannot explain the large value measured by the CDF collaboration.

\section{Acknowledgments}

We would like to thank Martin Beneke, Ulrich Haisch, Nikolaos Kidonakis, Kirill Melnikov, Sven Moch, Markus Schulze, and Berthold Stech for useful discussions. A. F. is grateful to the members of the Center for Cosmology and Particle Physics of New York University for their kind hospitality. This research was supported in part by the State of Rhineland-Palatinate via the Research Centre Elementary Forces and Mathematical Foundations, by the Helmholtz Institute Mainz, and by the German Federal Ministry for Education and Research under grant 05H09UME: Precision Calculations for Collider and Flavour Physics at the LHC.

\section{A RG-evolution factors and anomalous dimensions}

Here we collect the expressions of the perturbative solutions to NNLL order for the RG factors $S, a_{\gamma}$, and $\boldsymbol{u}$ in (83) and (84), which appear in the solution of the evolution matrix $\boldsymbol{U}$ in the final resummed result (92). We also collect the expansion coefficients of the anomalous dimensions and QCD $\beta$-function needed at this order.

We first define expansion coefficients of the anomalous dimensions and QCD $\beta$-function as

$$
\begin{aligned}
\Gamma_{\text {cusp }}\left(\alpha_{s}\right) & =\Gamma_{0} \frac{\alpha_{s}}{4 \pi}+\Gamma_{1}\left(\frac{\alpha_{s}}{4 \pi}\right)^{2}+\Gamma_{2}\left(\frac{\alpha_{s}}{4 \pi}\right)^{3}+\ldots, \\
\beta\left(\alpha_{s}\right) & =-2 \alpha_{s}\left[\beta_{0} \frac{\alpha_{s}}{4 \pi}+\beta_{1}\left(\frac{\alpha_{s}}{4 \pi}\right)^{2}+\beta_{2}\left(\frac{\alpha_{s}}{4 \pi}\right)^{3}+\ldots\right],
\end{aligned}
$$

and similarly for the other anomalous dimensions (recall that $\Gamma_{\text {cusp }}=C_{F} \gamma_{\text {cusp }}$ for the $q \bar{q}$ channel, and $\Gamma_{\text {cusp }}=C_{A} \gamma_{\text {cusp }}$ for the $g g$ channel). In terms of these quantities, the function $a_{\Gamma}$ is given by $[63,106]$

$$
a_{\Gamma}(\nu, \mu)=\frac{\Gamma_{0}}{2 \beta_{0}}\left\{\ln \frac{\alpha_{s}(\mu)}{\alpha_{s}(\nu)}+\left(\frac{\Gamma_{1}}{\Gamma_{0}}-\frac{\beta_{1}}{\beta_{0}}\right) \frac{\alpha_{s}(\mu)-\alpha_{s}(\nu)}{4 \pi}\right\},
$$

and the result for the Sudakov factor $S$ reads

$$
\begin{array}{r}
S(\nu, \mu)=\frac{\Gamma_{0}}{4 \beta_{0}^{2}}\left\{\frac{4 \pi}{\alpha_{s}(\nu)}\left(1-\frac{1}{r}-\ln r\right)+\left(\frac{\Gamma_{1}}{\Gamma_{0}}-\frac{\beta_{1}}{\beta_{0}}\right)(1-r+\ln r)+\frac{\beta_{1}}{2 \beta_{0}} \ln ^{2} r\right. \\
+\frac{\alpha_{s}(\nu)}{4 \pi}\left[\left(\frac{\beta_{1} \Gamma_{1}}{\beta_{0} \Gamma_{0}}-\frac{\beta_{2}}{\beta_{0}}\right)(1-r+r \ln r)+\left(\frac{\beta_{1}^{2}}{\beta_{0}^{2}}-\frac{\beta_{2}}{\beta_{0}}\right)(1-r) \ln r\right.
\end{array}
$$




$$
\left.\left.-\left(\frac{\beta_{1}^{2}}{\beta_{0}^{2}}-\frac{\beta_{2}}{\beta_{0}}-\frac{\beta_{1} \Gamma_{1}}{\beta_{0} \Gamma_{0}}+\frac{\Gamma_{2}}{\Gamma_{0}}\right) \frac{(1-r)^{2}}{2}\right]\right\}
$$

where $r=\alpha_{s}(\mu) / \alpha_{s}(\nu)$.

The matrix equation for $\boldsymbol{u}$ can be evaluated using the techniques described in $[107,108]$. To this end, we first define the matrix $\boldsymbol{V}$ which diagonalizes $\gamma^{h(0)}$ as

$$
\gamma_{D}^{h(0)}=\boldsymbol{V}^{-1} \gamma^{h(0)} \boldsymbol{V}
$$

We also define the vector $\vec{\gamma}^{h(0)}$ consisting of the diagonal elements of $\boldsymbol{\gamma}_{D}^{h(0)}$. Then the solution at NNLL reads

$$
\boldsymbol{u}\left(M, m_{t}, \cos \theta, \mu_{h}, \mu\right)=\boldsymbol{V}\left(1+\frac{\alpha_{s}(\mu)}{4 \pi} \boldsymbol{K}\right)\left(\left[\frac{\alpha_{s}\left(\mu_{h}\right)}{\alpha_{s}(\mu)}\right]^{\frac{\vec{\gamma}^{h(0)}}{2 \beta_{0}}}\right)_{D}\left(1-\frac{\alpha_{s}\left(\mu_{h}\right)}{4 \pi} \boldsymbol{K}\right) \boldsymbol{V}^{-1}
$$

where the matrix elements of $\boldsymbol{K}$ are

$$
K_{I J}=\delta_{I J} \vec{\gamma}_{I}^{h(0)} \frac{\beta_{1}}{2 \beta_{0}^{2}}-\frac{\left[\boldsymbol{V}^{-1} \boldsymbol{\gamma}^{h(1)} \boldsymbol{V}\right]_{I J}}{2 \beta_{0}+\vec{\gamma}_{I}^{h(0)}-\vec{\gamma}_{J}^{h(0)}} .
$$

Finally, we collect the expansion coefficients of the anomalous dimensions and QCD $\beta$ function needed in this work. The cusp anomalous dimension to three-loop order is [109]

$$
\begin{aligned}
\gamma_{0}^{\text {cusp }}= & 4 \\
\gamma_{1}^{\text {cusp }}= & \left(\frac{268}{9}-\frac{4 \pi^{2}}{3}\right) C_{A}-\frac{80}{9} T_{F} n_{f}, \\
\gamma_{2}^{\text {cusp }}= & C_{A}^{2}\left(\frac{490}{3}-\frac{536 \pi^{2}}{27}+\frac{44 \pi^{4}}{45}+\frac{88}{3} \zeta_{3}\right)+C_{A} T_{F} n_{f}\left(-\frac{1672}{27}+\frac{160 \pi^{2}}{27}-\frac{224}{3} \zeta_{3}\right) \\
& +C_{F} T_{F} n_{f}\left(-\frac{220}{3}+64 \zeta_{3}\right)-\frac{64}{27} T_{F}^{2} n_{f}^{2} .
\end{aligned}
$$

For the anomalous dimensions entering $\gamma^{h}$, we have [74,79]

$$
\begin{aligned}
& \gamma_{0}^{q}=-3 C_{F}, \\
& \gamma_{1}^{q}=C_{F}^{2}\left(-\frac{3}{2}+2 \pi^{2}-24 \zeta_{3}\right)+C_{F} C_{A}\left(-\frac{961}{54}-\frac{11 \pi^{2}}{6}+26 \zeta_{3}\right)+C_{F} T_{F} n_{f}\left(\frac{130}{27}+\frac{2 \pi^{2}}{3}\right), \\
& \gamma_{0}^{g}=-\frac{11}{3} C_{A}+\frac{4}{3} T_{F} n_{f}, \\
& \gamma_{1}^{g}=C_{A}^{2}\left(-\frac{692}{27}+\frac{11 \pi^{2}}{18}+2 \zeta_{3}\right)+C_{A} T_{F} n_{f}\left(\frac{256}{27}-\frac{2 \pi^{2}}{9}\right)+4 C_{F} T_{F} n_{f},
\end{aligned}
$$




$$
\begin{aligned}
& \gamma_{0}^{Q}=-2 C_{F} \\
& \gamma_{1}^{Q}=C_{F} C_{A}\left(-\frac{98}{9}+\frac{2 \pi^{2}}{3}-4 \zeta_{3}\right)+\frac{40}{9} C_{F} T_{F} n_{f},
\end{aligned}
$$

as well as $[54,55,74,110-112]$

$$
\begin{aligned}
\gamma_{0}^{\text {cusp }}(\beta)= & \gamma_{0}^{\text {cusp }} \beta \operatorname{coth} \beta \\
\gamma_{1}^{\text {cusp }}(\beta)= & \gamma_{1}^{\operatorname{cusp}} \beta \operatorname{coth} \beta+8 C_{A}\left\{\frac{\pi^{2}}{6}+\zeta_{3}+\beta^{2}\right. \\
& +\operatorname{coth}^{2} \beta\left[\operatorname{Li}_{3}\left(e^{-2 \beta}\right)+\beta \operatorname{Li}_{2}\left(e^{-2 \beta}\right)-\zeta_{3}+\frac{\pi^{2}}{6} \beta+\frac{\beta^{3}}{3}\right] \\
& \left.+\operatorname{coth} \beta\left[\operatorname{Li}_{2}\left(e^{-2 \beta}\right)-2 \beta \ln \left(1-e^{-2 \beta}\right)-\frac{\pi^{2}}{6}(1+\beta)-\beta^{2}-\frac{\beta^{3}}{3}\right]\right\} \\
g_{0}(\beta)= & 0, \\
g_{1}(\beta)= & \operatorname{coth} \beta\left[\beta^{2}+2 \beta \ln \left(1-e^{-2 \beta}\right)-\operatorname{Li}_{2}\left(e^{-2 \beta}\right)+\frac{\pi^{2}}{6}\right]-\beta^{2}-\frac{\pi^{2}}{6} .
\end{aligned}
$$

The anomalous dimensions of the PDFs are given by

$$
\begin{aligned}
\gamma_{0}^{\phi_{q}} & =3 C_{F}, \\
\gamma_{1}^{\phi_{q}} & =C_{F}^{2}\left(\frac{3}{2}-2 \pi^{2}+24 \zeta_{3}\right)+C_{F} C_{A}\left(\frac{17}{6}+\frac{22 \pi^{2}}{9}-12 \zeta_{3}\right)-C_{F} T_{F} n_{f}\left(\frac{2}{3}+\frac{8 \pi^{2}}{9}\right), \\
\gamma_{0}^{\phi_{g}} & =\frac{11}{3} C_{A}-\frac{4}{3} T_{F} n_{f}, \\
\gamma_{0}^{\phi_{g}} & =C_{A}^{2}\left(\frac{32}{3}+12 \zeta_{3}\right)-\frac{16}{3} C_{A} T_{F} n_{f}-4 C_{F} T_{F} n_{f},
\end{aligned}
$$

and for the QCD $\beta$-function to three-loop order we have

$$
\begin{aligned}
& \beta_{0}=\frac{11}{3} C_{A}-\frac{4}{3} T_{F} n_{f}, \\
& \beta_{1}=\frac{34}{3} C_{A}^{2}-\frac{20}{3} C_{A} T_{F} n_{f}-4 C_{F} T_{F} n_{f}, \\
& \beta_{2}=\frac{2857}{54} C_{A}^{3}+\left(2 C_{F}^{2}-\frac{205}{9} C_{F} C_{A}-\frac{1415}{27} C_{A}^{2}\right) T_{F} n_{f}+\left(\frac{44}{9} C_{F}+\frac{158}{27} C_{A}\right) T_{F}^{2} n_{f}^{2} .
\end{aligned}
$$




\section{References}

[1] Tevatron Electroweak Working Group and the CDF and D0 Collaborations, arXiv:0903.2503 [hep-ex].

[2] W. Bernreuther, J. Phys. G 35, 083001 (2008) [arXiv:0805.1333 [hep-ph]].

[3] R. Frederix and F. Maltoni, JHEP 0901, 047 (2009) [arXiv:0712.2355 [hep-ph]].

[4] V. Barger, T. Han and D. G. E. Walker, Phys. Rev. Lett. 100, 031801 (2008) [arXiv:hep$\mathrm{ph} / 0612016]$.

[5] U. Baur and L. H. Orr, Phys. Rev. D 77, 114001 (2008) [arXiv:0803.1160 [hep-ph]].

[6] T. Aaltonen et al. [CDF Collaboration], Phys. Rev. Lett. 100, 231801 (2008) [arXiv:0709.0705 [hep-ex]].

[7] T. Aaltonen et al. [CDF Collaboration], Phys. Rev. D 77, 051102 (2008) [arXiv:0710.5335 [hep-ex]].

[8] V. M. Abazov et al. [D0 Collaboration], Phys. Lett. B 668, 98 (2008) [arXiv:0804.3664 [hep-ex]].

[9] T. Aaltonen et al. [CDF Collaboration], Phys. Rev. Lett. 102, 222003 (2009) [arXiv:0903.2850 [hep-ex]].

[10] V. M. Abazov et al. [D0 Collaboration], Phys. Rev. Lett. 100, 142002 (2008) [arXiv:0712.0851 [hep-ex]].

[11] T. Aaltonen et al. [CDF Collaboration], Phys. Rev. Lett. 101, 202001 (2008) [arXiv:0806.2472 [hep-ex]].

[12] CDF Collaboration, CDF Public Report No. 9724 (2009).

[13] P. Nason, S. Dawson and R. K. Ellis, Nucl. Phys. B 303, 607 (1988).

[14] W. Beenakker, H. Kuijf, W. L. van Neerven and J. Smith, Phys. Rev. D 40, 54 (1989).

[15] W. Beenakker, W. L. van Neerven, R. Meng, G. A. Schuler and J. Smith, Nucl. Phys. B 351, 507 (1991).

[16] M. Czakon and A. Mitov, Nucl. Phys. B 824, 111 (2010) [arXiv:0811.4119 [hep-ph]].

[17] P. Nason, S. Dawson and R. K. Ellis, Nucl. Phys. B 327, 49 (1989) [Erratum-ibid. B 335, 260 (1990)].

[18] M. L. Mangano, P. Nason and G. Ridolfi, Nucl. Phys. B 373, 295 (1992).

[19] S. Frixione, M. L. Mangano, P. Nason and G. Ridolfi, Phys. Lett. B 351, 555 (1995) [arXiv:hep-ph/9503213]. 
[20] J. H. Kühn and G. Rodrigo, Phys. Rev. Lett. 81, 49 (1998) [arXiv:hep-ph/9802268].

[21] J. H. Kühn and G. Rodrigo, Phys. Rev. D 59, 054017 (1999) [arXiv:hep-ph/9807420].

[22] M. Czakon, A. Mitov and S. Moch, Phys. Lett. B 651, 147 (2007) [arXiv:0705.1975 [hep-ph]].

[23] M. Czakon, A. Mitov and S. Moch, Nucl. Phys. B 798, 210 (2008) [arXiv:0707.4139 [hep-ph]].

[24] M. Czakon, Phys. Lett. B 664, 307 (2008) [arXiv:0803.1400 [hep-ph]].

[25] R. Bonciani, A. Ferroglia, T. Gehrmann, D. Maitre and C. Studerus, JHEP 0807, 129 (2008) [arXiv:0806.2301 [hep-ph]].

[26] R. Bonciani, A. Ferroglia, T. Gehrmann and C. Studerus, JHEP 0908, 067 (2009) [arXiv:0906.3671 [hep-ph]].

[27] J. G. Korner, Z. Merebashvili and M. Rogal, Phys. Rev. D 77, 094011 (2008) [arXiv:0802.0106 [hep-ph]].

[28] C. Anastasiou and S. M. Aybat, Phys. Rev. D 78, 114006 (2008) [arXiv:0809.1355 [hep$\mathrm{ph}]$.

[29] B. Kniehl, Z. Merebashvili, J. G. Korner and M. Rogal, Phys. Rev. D 78, 094013 (2008) [arXiv:0809.3980 [hep-ph]].

[30] S. Dittmaier, P. Uwer and S. Weinzierl, Phys. Rev. Lett. 98, 262002 (2007) [arXiv:hep$\mathrm{ph} / 0703120]$.

[31] G. Sterman, Nucl. Phys. B 281, 310 (1987).

[32] S. Catani and L. Trentadue, Nucl. Phys. B 327, 323 (1989).

[33] E. Laenen, J. Smith and W. L. van Neerven, Nucl. Phys. B 369, 543 (1992).

[34] E. Laenen, J. Smith and W. L. van Neerven, Phys. Lett. B 321, 254 (1994) [arXiv:hep$\mathrm{ph} / 9310233]$.

[35] E. L. Berger and H. Contopanagos, Phys. Lett. B 361, 115 (1995) [arXiv:hep$\mathrm{ph} / 9507363]$.

[36] E. L. Berger and H. Contopanagos, Phys. Rev. D 54, 3085 (1996) [arXiv:hep$\mathrm{ph} / 9603326]$.

[37] E. L. Berger and H. Contopanagos, Phys. Rev. D 57, 253 (1998) [arXiv:hep-ph/9706206].

[38] S. Catani, M. L. Mangano, P. Nason and L. Trentadue, Phys. Lett. B 378, 329 (1996) [arXiv:hep-ph/9602208]. 
[39] R. Bonciani, S. Catani, M. L. Mangano and P. Nason, Nucl. Phys. B 529, 424 (1998) [Erratum-ibid. B 803, 234 (2008)] [arXiv:hep-ph/9801375].

[40] S. Moch and P. Uwer, Phys. Rev. D 78, 034003 (2008) [arXiv:0804.1476 [hep-ph]].

[41] M. Czakon and A. Mitov, Phys. Lett. B 680, 154 (2009) [arXiv:0812.0353 [hep-ph]].

[42] U. Langenfeld, S. Moch and P. Uwer, Phys. Rev. D 80, 054009 (2009) [arXiv:0906.5273 [hep-ph]].

[43] M. Beneke, P. Falgari and C. Schwinn, Nucl. Phys. B 828, 69 (2010) [arXiv:0907.1443 [hep-ph]].

[44] M. Czakon, A. Mitov and G. Sterman, Phys. Rev. D 80, 074017 (2009) [arXiv:0907.1790 [hep-ph]].

[45] M. Beneke, M. Czakon, P. Falgari, A. Mitov and C. Schwinn, arXiv:0911.5166 [hep-ph].

[46] N. Kidonakis and G. Sterman, Phys. Lett. B 387, 867 (1996).

[47] N. Kidonakis and G. Sterman, Nucl. Phys. B 505, 321 (1997) [arXiv:hep-ph/9705234].

[48] A. Banfi and E. Laenen, Phys. Rev. D 71, 034003 (2005) [arXiv:hep-ph/0411241].

[49] L. G. Almeida, G. Sterman and W. Vogelsang, Phys. Rev. D 78, 014008 (2008) [arXiv:0805.1885 [hep-ph]].

[50] N. Kidonakis, Phys. Rev. D 64, 014009 (2001) [arXiv:hep-ph/0010002].

[51] N. Kidonakis, E. Laenen, S. Moch and R. Vogt, Phys. Rev. D 64, 114001 (2001) [arXiv:hep-ph/0105041].

[52] N. Kidonakis and R. Vogt, Phys. Rev. D 68, 114014 (2003) [arXiv:hep-ph/0308222].

[53] N. Kidonakis and R. Vogt, Phys. Rev. D 78, 074005 (2008) [arXiv:0805.3844 [hep-ph]].

[54] A. Ferroglia, M. Neubert, B. D. Pecjak and L. L. Yang, Phys. Rev. Lett. 103, 201601 (2009) [arXiv:0907.4791 [hep-ph]].

[55] A. Ferroglia, M. Neubert, B. D. Pecjak and L. L. Yang, JHEP 0911, 062 (2009) [arXiv:0908.3676 [hep-ph]].

[56] V. Ahrens, A. Ferroglia, M. Neubert, B. D. Pecjak and L. L. Yang, Phys. Lett. B 687, 331 (2010) [arXiv:0912.3375 [hep-ph]].

[57] N. Kidonakis, G. Oderda and G. Sterman, Nucl. Phys. B 525, 299 (1998) [arXiv:hep$\mathrm{ph} / 9801268]$.

[58] N. Kidonakis, G. Oderda and G. Sterman, Nucl. Phys. B 531, 365 (1998) [arXiv:hep$\mathrm{ph} / 9803241]$. 
[59] E. Laenen, G. Oderda and G. Sterman, Phys. Lett. B 438, 173 (1998) [arXiv:hep$\mathrm{ph} / 9806467]$.

[60] S. Catani, M. L. Mangano and P. Nason, JHEP 9807, 024 (1998) [arXiv:hep$\mathrm{ph} / 9806484]$.

[61] J. C. Collins, D. E. Soper and G. Sterman, Adv. Ser. Direct. High Energy Phys. 5, 1 (1988) [arXiv:hep-ph/0409313].

[62] T. Becher and M. Neubert, Phys. Rev. Lett. 97, 082001 (2006) [arXiv:hep-ph/0605050].

[63] T. Becher, M. Neubert and B. D. Pecjak, JHEP 0701, 076 (2007) [arXiv:hep$\mathrm{ph} / 0607228]$.

[64] T. Becher, M. Neubert and G. Xu, JHEP 0807, 030 (2008) [arXiv:0710.0680 [hep-ph]].

[65] V. Ahrens, T. Becher, M. Neubert and L. L. Yang, Phys. Rev. D 79, 033013 (2009) [arXiv:0808.3008 [hep-ph]].

[66] V. Ahrens, T. Becher, M. Neubert and L. L. Yang, Eur. Phys. J. C 62, 333 (2009) [arXiv:0809.4283 [hep-ph]].

[67] T. Becher and M. D. Schwartz, JHEP 1002, 040 (2010) [arXiv:0911.0681 [hep-ph]].

[68] C. W. Bauer, S. Fleming, D. Pirjol and I. W. Stewart, Phys. Rev. D 63, 114020 (2001) [arXiv:hep-ph/0011336].

[69] C. W. Bauer, D. Pirjol and I. W. Stewart, Phys. Rev. D 65, 054022 (2002) [arXiv:hep$\mathrm{ph} / 0109045]$.

[70] M. Beneke, A. P. Chapovsky, M. Diehl and T. Feldmann, Nucl. Phys. B 643, 431 (2002) [arXiv:hep-ph/0206152].

[71] M. Neubert, Phys. Rept. 245, 259 (1994) [arXiv:hep-ph/9306320].

[72] S. Fleming, A. H. Hoang, S. Mantry and I. W. Stewart, Phys. Rev. D 77, 114003 (2008) [arXiv:0711.2079 [hep-ph]].

[73] C. W. Bauer and M. D. Schwartz, Phys. Rev. D 76, 074004 (2007) [arXiv:hep$\mathrm{ph} / 0607296]$.

[74] T. Becher and M. Neubert, Phys. Rev. D 79, 125004 (2009) [Erratum-ibid. D 80, 109901 (2009)] [arXiv:0904.1021 [hep-ph]].

[75] R. J. Hill and M. Neubert, Nucl. Phys. B 657, 229 (2003) [arXiv:hep-ph/0211018].

[76] T. Becher, R. J. Hill and M. Neubert, Phys. Rev. D 69, 054017 (2004) [arXiv:hepph/0308122].

[77] S. Catani and M. H. Seymour, Phys. Lett. B 378, 287 (1996) [arXiv:hep-ph/9602277]. 
[78] S. Catani and M. H. Seymour, Nucl. Phys. B 485, 291 (1997) [Erratum-ibid. B 510, 503 (1998)] [arXiv:hep-ph/9605323].

[79] T. Becher and M. Neubert, JHEP 0906, 081 (2009) [arXiv:0903.1126 [hep-ph]].

[80] J. A. M. Vermaseren, arXiv:math-ph/0010025.

[81] G. P. Korchemsky and G. Marchesini, Phys. Lett. B 313, 433 (1993).

[82] S. Frixione, M. L. Mangano, P. Nason and G. Ridolfi, Nucl. Phys. B 412, 225 (1994) [arXiv:hep-ph/9306337].

[83] V. N. Gribov and L. N. Lipatov, Sov. J. Nucl. Phys. 15, 438 (1972) [Yad. Fiz. 15, 781 (1972)].

[84] G. Altarelli and G. Parisi, Nucl. Phys. B 126, 298 (1977).

[85] Y. L. Dokshitzer, Sov. Phys. JETP 46, 641 (1977) [Zh. Eksp. Teor. Fiz. 73, 1216 (1977)].

[86] S. W. Bosch, B. O. Lange, M. Neubert and G. Paz, Nucl. Phys. B 699, 335 (2004) [arXiv:hep-ph/0402094].

[87] A. D. Martin, W. J. Stirling, R. S. Thorne and G. Watt, Eur. Phys. J. C 64, 653 (2009) [arXiv:0905.3531 [hep-ph]].

[88] J. M. Campbell and R. K. Ellis, Phys. Rev. D 62, 114012 (2000) [arXiv:hep-ph/0006304].

[89] A. Czarnecki and K. Melnikov, Phys. Rev. Lett. 80, 2531 (1998) [arXiv:hep-ph/9712222].

[90] M. Beneke, A. Signer and V. A. Smirnov, Phys. Lett. B 454, 137 (1999) [arXiv:hep$\mathrm{ph} / 9903260]$.

[91] A. Czarnecki and K. Melnikov, Phys. Rev. D 65, 051501 (2002) [arXiv:hep-ph/0108233].

[92] CDF Collaboration, CDF Public Report No. 9913 (2009).

[93] W. Bernreuther and Z. G. Si, arXiv:1003.3926 [hep-ph].

[94] O. Antunano, J. H. Kühn and G. Rodrigo, Phys. Rev. D 77, 014003 (2008) [arXiv:0709.1652 [hep-ph]].

[95] P. H. Frampton, J. Shu and K. Wang, Phys. Lett. B 683, 294 (2010) [arXiv:0911.2955 [hep-ph]].

[96] J. Shu, T. M. P. Tait and K. Wang, Phys. Rev. D 81, 034012 (2010) [arXiv:0911.3237 [hep-ph]].

[97] I. Dorsner, S. Fajfer, J. F. Kamenik and N. Kosnik, Phys. Rev. D 81, 055009 (2010) [arXiv:0912.0972 [hep-ph]]. 
[98] J. Cao, Z. Heng, L. Wu and J. M. Yang, Phys. Rev. D 81, 014016 (2010) [arXiv:0912.1447 [hep-ph]].

[99] Q. H. Cao, D. McKeen, J. L. Rosner, G. Shaughnessy and C. E. M. Wagner, arXiv:1003.3461 [hep-ph].

[100] V. M. Abazov et al. [D0 Collaboration], Phys. Rev. D 80, 071102 (2009) [arXiv:0903.5525 [hep-ex]].

[101] I. I. Y. Bigi, M. A. Shifman, N. G. Uraltsev and A. I. Vainshtein, Phys. Rev. D 50, 2234 (1994) [arXiv:hep-ph/9402360].

[102] M. Beneke and V. M. Braun, Nucl. Phys. B 426, 301 (1994) [arXiv:hep-ph/9402364].

[103] N. Gray, D. J. Broadhurst, W. Grafe and K. Schilcher, Z. Phys. C 48, 673 (1990).

[104] J. Fleischer, F. Jegerlehner, O. V. Tarasov and O. L. Veretin, Nucl. Phys. B 539, 671 (1999) [Erratum-ibid. B 571, 511 (2000)] [arXiv:hep-ph/9803493].

[105] K. G. Chetyrkin and M. Steinhauser, Nucl. Phys. B 573, 617 (2000) [arXiv:hep$\mathrm{ph} / 9911434]$.

[106] M. Neubert, Eur. Phys. J. C 40, 165 (2005) [arXiv:hep-ph/0408179].

[107] A. J. Buras, M. Jamin, M. E. Lautenbacher and P. H. Weisz, Nucl. Phys. B 370, 69 (1992) [Addendum-ibid. B 375, 501 (1992)].

[108] G. Buchalla, A. J. Buras and M. E. Lautenbacher, Rev. Mod. Phys. 68, 1125 (1996) [arXiv:hep-ph/9512380].

[109] S. Moch, J. A. M. Vermaseren and A. Vogt, Nucl. Phys. B 688, 101 (2004) [arXiv:hep$\mathrm{ph} / 0403192]$.

[110] G. P. Korchemsky and A. V. Radyushkin, Nucl. Phys. B 283, 342 (1987).

[111] G. P. Korchemsky and A. V. Radyushkin, Phys. Lett. B 279, 359 (1992) [arXiv:hep$\mathrm{ph} / 9203222]$.

[112] N. Kidonakis, Phys. Rev. Lett. 102, 232003 (2009) [arXiv:0903.2561 [hep-ph]]. 